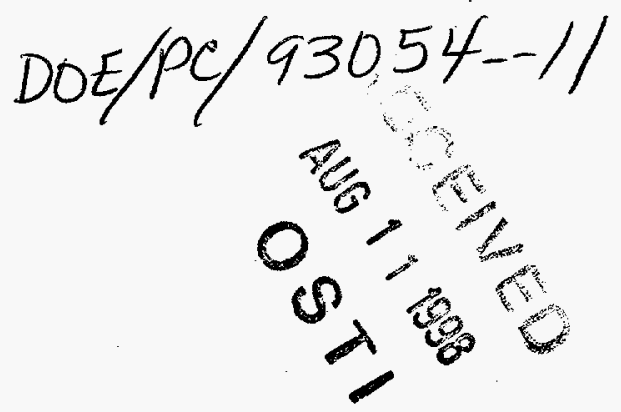

\title{
A Characterization and Evaluation of Coal Liquefaction Process Streams
}

\author{
Quarterly Report \\ January 1 - March 31, 1997 \\ By: \\ G. A. Robbins; S. D. Brandes \\ G. W. Heunisch; R. A. Winschel
}

Work Performed Under Contract No.: DE-AC22-94PC93054

For

U.S. Department of Energy

Office of Fossil Energy

Federal Energy Technology Center

P.O. Box 880

Morgantown, West Virginia 26507-0880

CONSOL, Inc.

4000 Brownsville Road

Library, Pennsylvania 15129

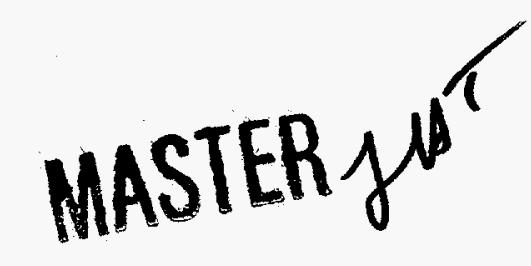

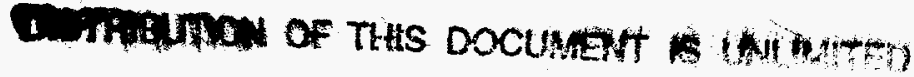




\section{Disclaimer}

This report was prepared as an account of work sponsored by an agency of the United States Government. Neither the United States Government nor any agency thereof, nor any of their employees, makes any warranty, express or implied, or assumes any legal liability or responsibility for the accuracy, completeness, or usefulness of any information, apparatus, product, or process disclosed, or represents that its use would not infringe privately owned rights. Reference herein to any specific commercial product, process, or service by trade name, trademark, manufacturer, or otherwise does not necessarily constitute or imply its endorsement, recommendation, or favoring by the United States Government or any agency thereof. The views and opinions of authors expressed herein do not necessarily state or reflect those of the United States Government or any agency thereof. 


\section{DISCLAIMER}

Portions of this document may be illegible electronic image products. Images are produced from the best available original document. 


\section{ABSTRACT}

This is the Technical Progress Report for the eleventh quarter of activities under DOE Contract No. DE-AC22-94PC93054. It covers the period January 1 through March 31, 1997. Described in this report are the following activities:

- $\quad$ CONSOL characterized process stream samples from HTI Run ALC-2, in which Black Thunder Mine coal was liquefied using four combinations of dispersed catalyst precursors. These results are described in the Results and Discussion section of this report.

- $\quad$ Oil assays were completed on the HTI Run PB-05 product blend. Background information is presented in the Results and Discussion section of this report. The results are presented in Appendix 1.

- Fractional distillation of the net product oil of HTI Run POC-1 was completed. Background information is presented in the Results and Discussion section of this report. The results are presented in Appendix 2.

- CONSOL completed an evaluation of the potential for producing alkylphenyl ethers from coal liquefaction phenols. Those results are described briefly in the Results and Discussion section of this report. The full report is presented in Appendix 3.

- At the request of DOE, various coal liquid samples and relevant characterization data were supplied to the University of West Virginia and the Federal Energy Technology Center. These activities are described in Appendix 4.

- The University of Delaware is conducting resid reactivity tests and is completing the resid reaction computer model. A summary of Delaware's progress is provided in the Results and Discussion section.

- The University of Delaware was instructed on the form in which the computer model is to be delivered to CONSOL (Appendix 5).

- The University of Delaware submitted a paper on the resid reactivity work for presentation at the 213th National Meeting of the American Chemical Society, April 13-17, 1997 in San Francisco, California. The paper, "Kinetics of Hydroprocessing of Coal-Derived Vacuum Resids", is appended (Appendix 6). 
TABLE OF CONTENTS

Page

EXECUTIVE SUMMARY

Characterization of Samples from HTI Run ALC-2

INTRODUCTION

Contract Overview

Contract Activities This Period

Activities in Progress

RESULTS AND DISCUSSION

Characterization of Samples from HTI Run ALC-2

\section{This page to be completed following DOE review}

CONCLUSIONS

REFERENCES

LIST OF ABBREVIATIONS AND ACRONYMS

\section{LIST OF APPENDICES}

\section{Appendix}

Page

1 Caleb Brett Report on Crude Oil Assay of Net Products of HTI Run PB-05

2 Caleb Brett Report on Fractional Distillation of Net Products of HRI Run POC-1

A1-1

3 Report on Preparation of Alkyl Aryl Ethers from Coal-Derived Phenols

4 Samples Supplied to Other DOE Projects

5 Format Request for Software of Kinetic/Mechanistic Model of Resid Reactivity

6 Manuscript, "Kinetics of Hydroprocessing of Coal-Derived Vacuum Resids" 


\section{Section 1 \\ EXECUTIVE SUMMARY}

\section{CHARACTERIZATION OF SAMPLES FROM HTI RUN ALC-2}

CONSOL characterized process stream samples from HTI Run ALC-2, in which Black Thunder Mine coal was liquefied using four combinations of dispersed catalyst precursors. The major conclusions from CONSOL's analyses of samples from HTI Run ALC-2 follow.

- $\quad$ Oil properties were nearly constant for the four run conditions. This consistency implies stability in the operation of the new distillation system and suggests that there was little difference in performance of the four catalyst systems tested.

- Coal and oil properties provided no insight into the reasons for solids separation problems with toluene extraction and pressure filtration observed during the run.

- Solids rejected during the first condition had high Ni concentrations, the origin of which (analytical artifact or trace contamination) is unknown.

- Most toluene-extracted solids (TES) and toluene-extracted oil (TEO) samples contained more than a few percent toluene, as received by CONSOL. CONSOL characterization confirmed generally poor extraction in the early part of the run. However, extraction appeared to be moderately successful in periods $3,9,12$, and 13 .

- The tetrahydrofuran (THF)-soluble fraction of the continuous vacuum still bottoms (CVSB) sample from Condition 4, period 21, was analyzed to determine if Ni or Mo was present in oil-soluble form. The occurrence of $\mathrm{Ni}$ and $\mathrm{Mo}$ in the THF-solubles is coincident with the occurrence of a trace amount of ash; thus, the $\mathrm{Ni}$ and Mo are not believed to be organically associated.

- The first-stage separator overhead (SOH1) oils and reduced-pressure still overhead (RPSOH) oils had very high concentrations (perhaps $25 \%$ ) of phenolics. They are potential sources of phenolic chemical feedstocks. 
- The properties of the hydrotreated product oils showed that the in-line hydrotreater (HTU) was effective for removal of phenolics and most of the aromatics.

- There appeared to be a recent change, relative to characteristics observed over the past three years, in the characteristics of HTI's L-814 oil used for run start-up and slurry oil make-up.

\section{CRUDE OIL ASSAY OF NET PRODUCTS OF HTI RUN PB-05}

At DOE's request, CONSOL arranged a crude oil assay on the net products of HTI Run PB-05 (also known as Run 227-97). The crude oil assay was conducted by Inchcape (now Intertek) Testing Services Caleb Brett Laboratory (Houston, TX) on the net products obtained during operating condition 6 of Run PB-05, in which Hondo resid was coprocessed with Illinois 6 coal. The sample was obtained when the on-line hydrotreater was by-passed.

\section{FRACTIONAL DISTILLATION OF NET PRODUCTS OF HRI RUN POC-1}

Inconsistencies in distillation data generated by Conoco and Southwesi Research Institute in 1995 on HTI net product oils prompted DOE to request additional tests. CONSOL provided a one-gallon sample of $\mathrm{HTI}$ Run POC-1 net product oil to Intertek Testing Services Caleb Brett Laboratory (Houston, TX) for fractional distillation. The results are presented in this report.

\section{PREPARATION OF ALKYL ARYL ETHERS FROM COAL-DERIVED PHENOLS}

CONSOL evaluated reactions to synthesize alkyl phenyl ethers from coal liquefaction phenols. The program included a literature review and laboratory chemical syntheses. An extensive literature search identified the Williamson Synthesis and its modifications as the preferred methods to produce mixed ethers from phenols. A variation of the Williamson Synthesis was used to produce phenetole and the ethyl derivatives of the phenolics from a caustic extract of a crude direct coal liquefaction product. Other approaches involving acid catalysts failed to produce ethers. It is recommended that the ethyl ether derivatives of coal liquefaction phenols be synthesized and characterized as a diesel fuel extender. CONSOL will develop a plan to extract phenols, synthesize the ethers and coordinate the fuel characterization upon DOE's approval. 


\section{RESID REACTIVITY}

The University of Delaware completed a parametric study with two resid samples. Temperature, pressure, residence time, catalyst loading, and resid concentration were explored. Conversion leveled off with time indicating the presence of a non-convertible component. This component was isolated by successive solvent-solubility extractions and subjected to hydrotreating. Conversion of the isolated material to $454^{\circ} \mathrm{C}^{-}\left(850^{\circ} \mathrm{F}^{-}\right)$material was only ca. $1 \mathrm{wt} \%$.

The structural molecular model was completed. The computer-generated molecular representations were found to match well the experimental data. The reaction model was assembled for catalytic upgrading of coal resids and is undergoing refinement.

\section{IECHNICAL TRANSFER}

The University of Delaware submitted a paper on the resid reactivity work for presentation at the 213th National Meeting of the American Chemical Society, April 13-17, 1997, in San Francisco, CA. The paper, "Kinetics of Hydroprocessing of Coal-Derived Vacuum Resids", is appended. 


\section{Section 2}

INTRODUCTION

This is the Technical Progress Report for the eleventh quarter of activities under DOE Contract No. DE-AC22-94PC93054. It covers the period January 1 through March 31, 1997.

\section{CONTRACT OVERVIEW}

The objectives of this project are to support the DOE direct coal liquefaction process development program and to improve the useful application of analytical chemistry to direct coal liquefaction process development. This project builds on work performed in DOE Contract No. DE-AC22-89PC89883. Independent analyses by well-established methods are obtained of samples produced in direct coal liquefaction processes under evaluation by DOE. Additionally, new analytical instruments and techniques to examine coal-derived samples are being evaluated. The data obtained from this study are used to guide process development and to develop an improved data base on coal and coal liquids properties. A sample bank, established and maintained for use in this project, is available for use by other researchers. The reactivity of the non-distillable resids toward hydrocracking at liquefaction conditions (i.e., resid reactivity) is being examined. From the literature and experimental data, a kinetic model of resid conversion is being constructed. Such a model will provide insights to improve process performance and the economics of direct coal liquefaction.

\section{CONTRACT ACTIVITIES THIS PERIOD}

- CONSOL characterized process stream samples from HTI Run ALC-2, in which Black Thunder Mine coal was liquefied using four combinations of dispersed catalyst precursors. The results are described in the Results and Discussion section of this report.

- Oil assays were completed on the HTI Run PB-05 product blend. Background information is presented in the Results and Discussion section of this report. The results are presented in Appendix 1.

- Fractional distillation of the net product oil of HTI Run POC-1 was completed. Background information is presented in the Results and Discussion section of this report. The results are presented in Appendix 2. 
- CONSOL completed an evaluation of the potential for producing alkylphenyl ethers from coal liquefaction phenols. Those results are described briefly in the Results and Discussion section of this report. The full report is presented in Appendix 3.

- At the request of DOE, various coal liquid samples and relevant characterization data were supplied to the University of West Virginia and the Federal Energy Technology Center. These activities are described in Appendix 4.

- The University of Delaware is conducting resid reactivity tests and is completing the resid reaction computer model. A summary of Delaware's progress is provided in the Results and Discussion section.

- The University of Delaware was instructed on the form in which the computer model is to be delivered to CONSOL (Appendix 5).

- The University of Delaware submitted a paper on the resid reactivity work for presentation at the 213th National Meeting of the American Chemical Society, April 13-17, 1997 in San Francisco, California. The paper, "Kinetics of Hydroprocessing of Coal-Derived Vacuum Resids", is appended (Appendix 6).

\section{ACTIVITIES IN PROGRESS}

- Characterization work is under way on samples from HTI Run PB-04 and work was begun on samples from PB-06.

- The development of a kinetic/mechanistic model of resid reactivity is continuing. 


\section{Section 3}

RESULTS AND DISCUSSION

\section{CHARACTERIZATION OF SAMPLES FROM HTI RUN ALC-2 RUN BACKGROUND}

In HTI Run ALC-2 (Run 227-100), ${ }^{1,2}$ the liquefaction performance of Black Thunder Mine coal was tested with four combinations of impregnated (dispersed) catalyst precursors. A new distillation system and solids separation scheme was employed during the run. Run ALC-2 consisted of 21 operating periods at four conditions. The run was carried out from November 24 through December 23, 1996. The run was planned to be made under six conditions; however, several problems occurred early in the run that decreased the number of conditions and catalysts that could be tested to four. Run conditions and yields for Run ALC-2 are provided in Table 1., The major variable was the catalyst system used. This included differences in the active metals and molybdenum precursors used. The water-soluble catalyst precursors were impregnated on the feed coal. Sulfiding agents for the catalysts were $\mathrm{H}_{2} \mathrm{~S}$ fed to the preheater/first-stage reactor at a rate of $2 \%$ of the MF coal, and di-tertiary-nonyl polysulfide (TNPS containing $37 \%$ S) fed to the second-stage reactor at a rate equivalent to an $\mathrm{H}_{2} \mathrm{~S}$ rate of $0.5 \%$ of the MF coal. Other components in the configuration used were a coil pretreater/preheater, two forced-back mixed liquefaction reactors with an interstage vapor/slurny separator, and a direct-coupled (in-line) product hydrotreater (HTU) of increased capacity. The total recycle to MF coal ratio was approximately 1.67 (ca. $37 \%$ coal concentration). The target recycle solids to MF coal ratio was 0.20 . During the run, the actual solids recycle ratio was variable, and averaged a higher value of about 0.30 for the work-up periods. To provide ash recycle, the bulk of the continuous vacuum still bottoms (CVSB) produced was recycled directly; only a fraction of the material was sent to solids separation to reject solids from the process.

HTI's results indicated that the ash balance was not good in any of the run conditions. As a result, it was concluded that operations were not at steady-state when the samples were taken and when the material balance work-ups were performed. This places uncertainty on the catalyst comparisons that are based on the run performance data. HTI reported, however, that the Mo and $\mathrm{Ni}$ catalyst combination provided the best overall results. They also found that catalyst choice had no effect on coal conversion and that the Mo/Ni combination seemed to provide the best resid conversion. HTI found no apparent detriment to elimination of $\mathrm{Fe}$ catalyst from the subbituminous coal liquefaction system. The distillation system gave satisfactory performance, 
but numerous problems were encountered with solids separation. Relative to results from Condition 1 of Run ALC-1, the MAF $\mathrm{C}_{4}-524^{\circ} \mathrm{C}$ yield in Run ALC-2 was lower by about $10 \%$ (due to about $6 \%$ lower resid conversion and $4 \%$ higher $C_{1}-C_{3}$ gas yields). The hydrogen consumption was also about $0.4 \%$ lower in Run ALC-2. It was thought that the high gas yields in Run ALC-2 may have been due to recycle of reduced pressure still overheads (RPSOH), an oil stream that is much lower-boiling than is desired for recycle. It became necessary to recycle RPSOH material when the preferred materials were not available.

The catalysts and active metal concentrations tested (reported on an MF coal basis) were: Condition 1 - ammonium heptamolybdate (AHM) plus ferrous sulfate (100 mg/kg as Mo, $1610 \mathrm{mg} / \mathrm{kg}$ as $\mathrm{Fe}$ ), Condition 2 - AHM (110 mg/kg as MO), Condition 3 - AHM plus nickel sulfate (92 mg/kg as Mo, $51 \mathrm{mg} / \mathrm{kg}$ as Ni), and Condition 4 - phosphomolybdic acid (PMA, $116 \mathrm{mg} / \mathrm{kg}$ as Mo, $3 \mathrm{mg} / \mathrm{kg}$ as $\mathrm{P}$ ), respectively. The distillation system consisted of a continuous vacuum still (CVS) and a reduced-pressure still (RPS). Because it can be operated with a slight vacuum, it is called a "reduced pressure still". The CVS provides a vacuum gas oil at an adjustable cut point ( $427^{\circ} \mathrm{C}$ was the cut point from Period 7 on during Run ALC-2). The RPS provides a net product distillate with a cut point that can be adjusted to higher than $343^{\circ} \mathrm{C}$. In Run ALC-2, the RPS was operated at a cut point of $316^{\circ} \mathrm{C}$ (from period 7 on) to give a distillate that was predominantly $343{ }^{\circ} \mathrm{C}$-. The increase in direct-coupled hydrotreater volume from $500 \mathrm{~cm}^{3}$ used in Run ALC-1 to $800 \mathrm{~cm}^{3}$ in Run ALC-2 provided highly effective product hydrotreating. Nitrogen concentrations of about $40 \mathrm{mg} / \mathrm{kg}$ or less, and sulfur concentrations of about $60 \mathrm{mg} / \mathrm{kg}$ or less were obtained. The solids separation scheme was designed to avoid pressure filtration bottlenecks by using toluene extraction to recover resid and oil directly from CVS bottoms (CVSB) material. However, toluene extraction performed poorly with this material. In Condition 3 , pressure filtration was used for a few days until the erratic filtration performance became unmanageable. The solids separation method was changed near the end of Condition 3 to batch vacuum distillation at $524^{\circ} \mathrm{C}$ atmospheric equivalent endpoint, without toluene extraction. Solids separation was continued in this manner until the end of the run.

\section{BRIEF DESCRIPTION OF PLANT}

The run was performed in HTl's bench unit 227. Fresh feed coal was mixed batchwise with process recycle materials in a tank and transferred to a feed slurry tank that continuously fed the slurry to the liquefaction process. The feed slurry was fed to a preheater, which also conditioned the dispersed catalyst. Next, the slurry was fed to two successive stages of liquefaction. No 
supported catalysts were used in the liquefaction reactors; only disposable dispersed catalysts were used. A high-pressure separator after the first reactor allows light products to be taken off and the hydrogen concentration to be increased in the second reactor. The first-stage oil, called the first-stage separator overhead oil (designated here as $\mathrm{SOH} 1$ ), is sent with second-stage light oils and light distillate to an in-line fixed-bed hydrotreater. The in-line hydrotreater (or HTU) upgrades the product using the liquefaction reactor system off gases. The second stage of liquefaction is followed by high- and low-pressure separators. The separator overheads are fed to the in-line product hydrotreater, and the separator bottoms to distillation. The distillate (ca. IBP- $371^{\circ} \mathrm{C}$ ) is sent to the product hydrotreater, and the resid is extracted (i.e., toluene-washed) to provide a liquid for recycle and solids to reject ash. The hydrotreated product oil is called the second-stage separator overhead oil (designated here as $\mathrm{SOH}$ ).

Thirty-two oil samples and four feed coal samples were received from $\mathrm{HTI}$ for characterization. Major streams analyzed included the feed coal, the L-814 start-up oil, the unhydrotreated firststage separator overhead ( $\mathrm{SOH} 1$ ) oil, the separator bottoms (0-6 bottoms, the flashed liquefaction product), the continuous vacuum still bottoms (CVSB), the reduced-pressure still overheads (RPSOH), the reduced-pressure still bottoms (RPSB), the toluene-extracted solids (TES), the toluene-extracted oil (TEO), and the hydrotreated net product oil (SOH2).

\section{ANALYTICAL STRATEGY}

In the analytical work performed by CONSOL, an attempt was made to address the following questions:

- What caused the problems encountered in toluene extraction and pressure filtration during the run?

- What can be learned about changes in catalyst concentration on startup?

- What do sample characteristics say about:

- Catalyst performance differences?

- Equipment performance (e.g., distillation system)?

In order to address these questions, the characterization work was focused primarily on the feed coal, $0-6$ bottoms, CVSB, TES, and TEO stream samples, and on product distillate features and 
differences. Specifically investigated were the treated coal characteristics, the preasphaltenes content and characteristics of the CVSB resid, and the catalyst metals in the TES samples from the first condition.

\section{ANALYSIS OF FEED COALS}

Ten-pound samples were obtained of each of the four catalyst-treated Black Thunder coals used in Run ALC-2 (L-902, L-904, L-906, and L-911). The samples, which were shipped in 5-gallon pails, were stored in plastic bags after receipt. Analyses of the feed coals from Run ALC-2 were conducted with several goals in mind. Since ultra-fine particulate matter (i.e., particles smaller than $2.5 \mu \mathrm{m}$ diameter) was suspected to have caused the poor performance in filtration and toluene extraction, the size distribution of the feed coal was determined. Another analysis goal was to evaluate the overall characteristics of the coals, since the coals were to be used as a feedstock during agglomeration testing in preparation for Run ALC-3. Complete coal analyses were obtained, and their suitability as agglomeration feeds was evaluated.

Coal size distribution was determined by wet screen analysis. To examine the finest particles, the -325 mesh fraction from wet screening was analyzed by a Malvern laser scattering instrument. The coal size distribution (Table 2 ) shows that the coals are not unusually fine, and that they do not differ much in size consist. The wet screen analyses shows that the coals are $45-55 \%+200$ mesh and $25-35 \%-325$ mesh. There are minor differences among the samples of about $10 \%$ of the material in the +200 mesh fraction and the -325 mesh fraction. These differences may reflect treatment time in the ribbon blender, since the coals treated with $\mathrm{Ni}$ or $\mathrm{Fe}$ in addition to Mo spent additional time in the blender. The $d_{50}$ of the -325 mesh fractions were consistent at 28-30 $\mu \mathrm{m}$. The $d_{10}$ and $d_{50}$ of the -325 mesh fraction of these treated coals are similar to those of a whole Wilsonville feed (see footnote $b$ in Table 2). Thus, there is no indication of any unusual amount of ultra-fine material in these coals.

Other coal analyses (Table 2) included proximate and ultimate analyses, heating value, and major and trace ash element analyses. The results do not indicate anything unusual about these coals and, together with the size analyses, indicate that the coals are suitable agglomeration feedstocks. CONSOL's coal analyses were compared with those reported by HTI. ${ }^{1}$ Relative to $\mathrm{HTI}$ analyses for the feed coals, CONSOL obtained results that were in generally good agreement on moisture (within 0.2 wt \%), MF ash (within 0.1 wt \%), MF carbon (within $0.7 w t \%$, CONSOL typically higher by $\sim 0.6 \mathrm{wt} \%$ ), MF hydrogen (CONSOL was consistently $\sim 0.32 \mathrm{wt} \%$ lower), MF 
nitrogen (CONSOL was consistently $\sim 0.05$ wt $\%$ higher), MF sulfur (CONSOL was consistently 0.1-0.2 wt \% higher); and MF oxygen by difference (CONSOL was typically $\sim 0.5$ wt \% lower).

The $\mathrm{HTI}$ and CONSOL analyses of the catalyst element concentrations in the ash are provided in Table 3. Relative to $\mathrm{HTI}$ analyses, ${ }^{1}$ CONSOL results were slightly lower for Mo (0.13-0.15 wt \% for CONSOL vs. 0.15-0.18 wt \% for HTI), higher for Fe (3.77-6.41 wt \% for CONSOL vs. 3.18$5.25 \mathrm{wt} \%$ for $\mathrm{HTI}$ ), and slightly lower for Ni (49-750 mg/kg for CONSOL vs. $49-829 \mathrm{mg} / \mathrm{kg}$ for $\mathrm{HTI}$ ). Although there are some systematic differences in concentrations reported by CONSOL and $H T I$, the agreement between the CONSOL and $H T I$ analyses was quite good. These results do not suggest a reason for the poor extraction and filtration of the product. They do indicate that the coals have normal properties and size distribution and, thus, are suitable for developmental agglomeration tests in preparation of Run ALC-3.

\section{ANALYSIS OF CONDITION 1 TOLUENE-EXTRACTED SOLIDS FOR CATALYTIC METALS} Selected CONSOL analyses of toluene-extracted solids (TES) are presented in Table 4. Relative to CONSOL analyses for the major elemental concentrations in the ash from of the treated feed coal L-902 (ash $\mathrm{SO}_{3}$-free), $\mathrm{CONSOL}$ obtained results from TES sample ash (normalized $\mathrm{SO}_{3}$ - and $\mathrm{K}_{2} \mathrm{O}$-free) from Condition 1 that were: high for $\mathrm{Na}, \mathrm{Ca}, \mathrm{Mg}$, and $\mathrm{Ti}$, slightly high for $\mathrm{P}$, about the same for $\mathrm{Al}$, quite low for $\mathrm{Si}$, and not consistent for $\mathrm{Fe}$. For the catalytic elements (Table 4), TES analyses varied significantly, but the averages are in the expected range for $\mathrm{Mo}$ and $\mathrm{Fe}$. The concentration of $\mathrm{Ni}$ in the TES ash is much higher than in the feed coal ash. These results indicate that there is an unidentified source of $\mathrm{Ni}$ in the TES ash. Possible sources include vessel erosion, some other type of contamination, or analytical error. The analysis procedure is not expected to cause such an artifact, but further investigation is needed if the source of nickel contamination must be identified. Unfortunately, there are no HTI data on Ni concentrations in the continuous vacuum still bottoms (CVSB) samples from the early portion of the run (Table 3 ).

The concentrations of Fe measured by HTI in the CVSB sample ash (Table 3) were 28-58\% higher than the concentrations in the feed coal ash for each corresponding operating condition. The concentration of $\mathrm{Ni}$ measured by $\mathrm{HTI}$ in the CVSB ash was $24 \%$ lower than the corresponding concentration measured in the feed coal ash for Condition 3 . Thus, the HTI data indicate that the Ni concentration may never have reached its target in the circulating oil. 
Substantial variation in MO and Fe concentration in periods $3 B$ and $5 A B$ (Table 3) appeared to result from a feed disruption that occurred in period 4.

\section{EFFICIENCY OF TOLUENE WASHING}

Table 5 and Figure 1 show that the toluene content of whole TES and TEO samples, as received by CONSOL, ranged from 2 to $70 \%$. The toluene content was measured by heating the samples in a vacuum oven at $60^{\circ} \mathrm{C}$ and vacuum (ca. $5 \mathrm{~mm} \mathrm{Hg}$ ), until constant weight was obtained (up to several days). HTI personnel reported that these samples contained up to $8 \% 650^{\circ} \mathrm{F}^{-}$oils; some of this material may have been reported as toluene using CONSOL's procedure. ${ }^{2}$ Nevertheless, the small amount of $650{ }^{\circ} \mathrm{F}^{-}$oil and the generally high amount of material evaporated show that the evaporated material is predominently toluene. Table 5 and Figure 2 shows the concentrations of THF solubles, IOM, and ash measured by CONSOL on these samples, reported on a toluene-free basis. These results confirmed generally poor extraction in the early part of run, as evidenced by low concentrations of insolubles in the toluene-free TES samples. However, extraction was moderately successful in period 12 , as indicated by $56 \%$ insolubles in the TES, and a low concentration of insolubles in the TEO (assuming that the period 12 TEO sample was good). Extraction also may have been moderately successful in periods 3 , 9 , and 13 (the TES samples contained ca. $50 \%$ or more insolubles).

\section{CHARACTERISTICS OF 0.6 BOTTOMS AND CVSB SAMPLES}

Figures 3-7 and Tables 6-8 show that the characteristics of the 0-6 bottoms and CVSB samples varied little between the four conditions used in Run ALC-2. Characteristics that were unchanged include microautoclave coal conversions (Table $6, \mathrm{ca} .82-85 \%$ in $0-6$ bottoms $454^{\circ} \mathrm{C}$ - distillates), hydrogen distributions (Table 7 and Figures 3 and 7, e.g., ca. 31\% aromatic hydrogen in the filtered whole $0-6$ bottoms samples, and $\mathrm{ca}$. $36 \%$ aromatic hydrogen in the filtered whole CVSB samples), and phenolic $-\mathrm{OH}$ concentration $(0.9-1.2 \mathrm{meg} / \mathrm{g}$, Table 8 and Figure 4. The concentration of insoluble organic matter (IOM) in the CVSB and O-6 bottoms samples also was steady (6.6-8.9\% in 0-6 bottoms, $10.0-14.2 \%$ in CVSB). However, the Period 7 samples were low in ash concentration, and the Period 12 CVSB sample was high in ash concentration, relative to their Period 17 and Period 21 counterparts. This may be a consequence of the poor ash balances reported by HTI and LDP Associates for the run. ${ }^{1.3}$ in the CVSB samples, the concentration of THF solubles was inversely related to the ash concentration. These results show no differences that are attributable to catalyst differences between the four run conditions represented by samples from Periods $7,12,17$, and 21 . 
In order to investigate differences in preasphaltene amounts and characteristics, the THF solubles from the CVSB samples were separated by solvent fractionation into oils, asphaltenes, and preasphaltenes (Table 6 and Figure 6 ) using CONSOL's preparative liquid column fractionation (LCF) method. An attempt was made to characterize the collected preasphaltene fraction from one sample by proton NMR spectrometry and by FTIR spectroscopy, but there was insufficient material to obtain any useful information. Since the CVSB THF-soluble material from all four conditions consistently contained ca. $8-10 \%$ preasphaltenes, no differences were found that would be helpful in discovering the cause of the extraction and filtration problems. The only differences that were observed in these data (Figure 6, Table 6) were a higher concentration of the oils ( $78 \%$ vs. $67-70 \%$ ), and a lower concentration of the asphaltenes ( $14 \%$ vs. $21-22 \%$ ) in the Condition 4 CVSB THF-solubles, relative to the corresponding samples from the other conditions. Perhaps this difference was a consequence of use of the batch vacuum still for solids separation during Condition 4 , since more resid may have been rejected from the process.

In general, most sample characteristics changed little over the course of the run. Thus, there are no period-to-period differences that would suggest a reason for extraction/filtration problems. Furthermore, these results provide no conclusive indication of catalyst performance differences. Combined with the distillate product characteristics, the consistent results at each condition also indicate that distillation system operations and performance were very stable.

\section{DISTILLATE PRODUCT CHARACTERISTICS}

Characteristics of the distillate and light oil streams (first-stage (unhydrotreated) $\mathrm{SOH}$, secondstage (hydrotreated) SOH, RPSOH, and RPSB oils) are provided in Tables 7 and 8 and Figures 8-13. The phenolic $-\mathrm{OH}$ concentration and hydrogen distribution of these streams changed little over the run. This implies stability in the distillation system and provides no indication of performance differences between the catalysts used in the four run conditions.

The high phenolic - $\mathrm{OH}$ concentrations of the first-stage $\mathrm{SOH}$ and RPSOH oil samples (Table 8, Figures 8 and 11 ) indicate that these streams contain a significant fraction (perhaps $25 \%$ ) of phenolics. The phenolics are potential sources of relatively valuable chemical feedstocks. The characteristics of the hydrotreated second-stage $\mathrm{SOH}$ oil samples relative to those of the unhydrotreated first-stage $\mathrm{SOH}$ oil samples indicate that the in-line hydrotreater effectively removes phenolic $-\mathrm{OH}$ and most aromatic hydrogen from the product oils (Tables 7 and 8 , Figures 8-10). 


\section{NATURE OF Ni IN CONDITION 4 CVSB SAMPLE}

In HTI Run ALC-2, Ni-impregnated coal was fed only during Condition 3. The Ni concentrations in the continuous vacuum still bottoms (CVSB) samples taken during the run increase throughout Condition 3, then decrease slowly (but never to baseline) during Condition 4. There was speculation that the slow rate of decline of $\mathrm{Ni}$ concentration could result from recycle of organically associated $\mathrm{Ni}$ species. To help evaluate this issue, the THF-soluble fraction of the CVSB sample from condition 4, period 21, was analyzed to determine if $\mathrm{Ni}$ or Mo was present in oil-soluble form. The THF-solubles were prepared from the CVSB sample by pressure filtration with THF through a Whatman 42 paper (retains particles $>2.5 \mu \mathrm{m}$ ), and evaporation of the THF from the filtrate. An analysis gave $72.3 \%$ THF solubles, $11.0 \% 10 \mathrm{M}$, and $16.7 \%$ ash. The THFsoluble material contained $6 \mathrm{mg} / \mathrm{kg} \mathrm{Ni}, 4 \mathrm{mg} / \mathrm{kg} \mathrm{Mo}$, a total of $416 \mathrm{mg} / \mathrm{kg}$ of the oxides of $\mathrm{Si}, \mathrm{Al}$, $\mathrm{Fe}, \mathrm{Ca}$, and $\mathrm{Mg}$, and less than $0.1 \%$ ash (obtained using a separate aliquot of THF-soluble material). Thus, the occurrence of $\mathrm{Ni}$ and $\mathrm{Mo}$ is coincident with the occurrence of a trace amount of ash, and the Ni and Mo are presumed to be inorganically associated. The amount of Ni or Mo in the ash of the solubles (about $1 \%$ of the ash in the CVSB THF-solubles) is higher than in the ash of the coal or ashy process streams (about $600 \mathrm{mg} / \mathrm{kg}$ or less for $\mathrm{Ni}, 0.2 \%$ for $\mathrm{Mo}$ ).

\section{L-814 START-UP OIL: CHANGE OF CHARACTERISTICS FROM PRIOR SAMPLES}

HTI's L-814 (Tank 4) oil is used for run start-up and sometimes for make-up oil. The original inventory was a fluid catalytic cracking (FCC) decant oil. The characteristics of the inventory change over time from the exchange of oils between Tank 4 and the HTI operating units during start-up, make-up, and shut-down operations. Over the past several years, CONSOL has routinely requested a start-up oil sample from each run from which other process oils were collected for characterization. Characterization data were usually reported with data from other oils, although remarks were rarely made about the start-up oil characteristics. It was observed that the characteristics of the L-814 start-up oil sample collected from Run ALC-2 were different from those of samples collected over the prior three years.

The proton distributions of L-814 oil (Tank 4 oil) used in HTI Runs POC-2, CMSL-9, ALC-1, and ALC-2 were compared (Table 9). The most recent sample, from Run ALC-2, was less aromatic and more paraffinic than the earlier samples. The Run ALC-2 sample also was apparently lower in viscosity than the one from Run CMSL-9 that was visually inspected for this property. CONSOL requested an even more recent sample that was provided by HTI (sample ca. 6/30/97). Aromatic hydrogen content of the L-814 oils (Table 9) was $23.0 \%$ for Run POC-2, 20.4-23.3\% for 
Run CMSL-9, 20.6\% for Run ALC-1, $14.8 \%$ for Run ALC-2, and $8.0 \%$ in the $6 / 30 / 97$ sample. Alkyl beta aliphatic hydrogen content of the samples were $21.4 \%$ for Run POC-2, 23.8-25.4\% for Run CMSL-9, 24.0\% for Run ALC-1, 32.3\% for Run ALC-2, and $38.3 \%$ in the $6 / 30 / 97$ sample. The 6/30/97 sample confirms that the L-814 oil became (through replacement of the original oil by oils produced in HTI's tests) less aromatic and more paraffinic than its predecessors. Although the latest sample is the least aromatic and most paraffinic of those analyzed by CONSOL, its viscosity appears normal for this type of sample, in contrast with the Run ALC-2 sample, which had an unusually low viscosity. 


\section{CRUDE OILASSAY OF NET PRODUCTS OF HTI RUN PB-05}

CONSOL arranged to have a crude oil assay conducted on the net products of HTI Run PB-05 (also known as Run 227-97), at DOE's request. The crude oil assay was conducted on the net products obtained during operating condition 6 of Run PB-05 in which Hondo resid was coprocessed with Illinois 6 coal and the on-line hydrotreater was by-passed.

HTI provided CONSOL with four partially-filled 1-gallon containers of separator overhead samples from HTI Run PB-05, condition 6. The individual separator overhead samples were from periods 22A, 23A, 24A, and 25A. CONSOL prepared and blended these materials to produce a large sample for crude oil assay testing. The samples were decanted and filtered over ReeveAngel 802 filter paper to omit the small quantities of water and sediment, and then blended to produce a $12.99 \mathrm{~kg}$ sample (approximately 3.8 to $4 \mathrm{gal}$ ). The blended material was placed in a steel $5 \mathrm{gal}$ can and shipped to the Inchcape (now Intertek) Testing Services Caleb Brett Laboratory in Houston, TX, for the crude oil assay testing. The analytical test plan was prepared in conjunction with P. Zhou of Burns and Roe Services Corp. A vial of the final blend was retained by CONSOL. Caleb Brett's report appears as Appendix 1. 


\section{ERACTIONAL DISTILLATION OF NET PRODUCTS OF HRI RUN POC-1}

At DOE's request, CONSOL arranged to have various distillations conducted on the net products of HRI Run POC-1. DOE was interested in obtaining the distillation data on this material because

of inconsistencies between the fractional distillation data generated in 1995 by Conoco ${ }^{4}$ and Southwest Research Institute on different aliquots of the same sample of the net product of HTI Run POC-1. DOE wanted the distillations (fractional, ASTM D86, and simulated) re-checked. A one-gallon sample of the net product of HTI Run POC-1 (the whole crude oil) was recovered from the CONSOL sample bank. This sample was retumed to CONSOL after Conoco conducted the crude oil assay on the material. The sample was shipped to the Inchcape (now Intertek) Testing Services Caleb Brett Laboratory in Houston, TX, for the distillation testing. The analytical test plan was prepared in conjunction with P. Zhou of Burns and Roe Services Corp. Caleb Brett's report appears as Appendix 2. 


\section{PREPARATION OF ALKYL ARYL ETHERS FROM COAL-DERIVED PHENOLS}

CONSOL R\&D evaluated reactions to synthesize alkyl phenyl ethers from coal liquefaction phenols. The program included a literature review and laboratory chemical syntheses. Results are briefly presented here. The full report is provided in Appendix 3.

Phenols are produced during the direct liquefaction of coal and must be removed prior to producing transportation fuels. The crude liquefaction product is commonly hydrotreated to remove the phenols and other unsaturated components. An alternative method is to extract the phenols from the crude coal liquefaction product and use them in other commercial processes. Hydrogen consumption for the hydrotreatment of the liquefaction products would be reduced. The phenolic material could be converted to alkylphenyl ethers, which may be useful as fuel extenders. If the fuel extenders can be produced using grain alcohol, the products may be entitled to special tax considerations.

An extensive literature search identified the Williamson Synthesis and its modifications as the preferred methods to produce mixed ethers from phenols. A variation of the Williamson Synthesis was used to produce phenetole and the ethyl derivatives of the phenolics from a caustic extract of a crude direct coal liquefaction product. Other approaches involving acid catalysts failed to produce ethers.

It is recommended that the ethyl ether derivatives of coal liquefaction phenols be synthesized and characterized as a diesel fuel extender. CONSOL will develop a plan to extract the phenols, synthesize the ethers, and coordinate the fuel characterization upon DOE's approval. 


\section{RESID REACTIVITY}

The University of Delaware completed a parametric reaction study with two resid samples. Temperature, pressure, residence time, catalyst loading, and resid concentration were explored. The two samples chosen for the study (Wilsonville Run 260 V131B and Wilsonville Run 259 V131B) gave the highest ( $44.6 \mathrm{wt} \%$ ) and lowest (33.1 wt \%) resid conversions, respectively at the standard conditions of $420^{\circ} \mathrm{C}, 30 \mathrm{~min}, 3: 1$ tetralin:resid and $3 \mathrm{wt} \%$ molybdenum naphthenate catalyst. Figure 14 shows data for two temperatures and four residence times for each of the resids. The leveling off of conversion in time is consistent with the presence of a refractory resid component, which is difficult to convert to lower-boiling product.

The refractory component was isolated by successive solvent-solubility extractions and subjected to hydrotreating. The resid was first extracted in room temperature tetralin, and placed in a Soxhlet extractor and extracted in tetralin at reflux for $48 \mathrm{~h}$. The residue was dried and then hydrotreated at $440^{\circ} \mathrm{C}, 30 \mathrm{~min}$, with $3 \mathrm{wt} \% \mathrm{Mo}$, in $3: 1$ tetralin:residue. Conversion to $454^{\circ} \mathrm{C}^{-}$ $\left(850^{\circ} \mathrm{F}^{-}\right)$was only ca. $1 \mathrm{wt} \%$. The unconverted material represents about $15 \mathrm{wt} \%$ of the $454^{\circ} \mathrm{C}^{+}$ $\left(850^{\circ} \mathrm{F}^{+}\right)$resid. Elemental analyses of the successive insoluble fractions and the unconverted $454^{\circ} \mathrm{C}^{+}\left(850^{\circ} \mathrm{F}^{+}\right)$hydrotreated fraction are given in Table 10 . The $\mathrm{H} / \mathrm{C}$ molar ratio for the unconverted $454^{\circ} \mathrm{C}^{+}\left(850^{\circ} \mathrm{F}^{+}\right)$hydrotreated fraction is only 0.42 , roughly half that of the $454^{\circ} \mathrm{C}^{+}$ $\left(850^{\circ} \mathrm{F}^{+}\right)$resid.

All necessary information was determined for the Delaware set of $454^{\circ} \mathrm{C}^{+}\left(850^{\circ} \mathrm{F}^{+}\right)$coal resids to assemble a molecular model. The model was modified several times so that the computergenerated molecular representations match the experimental data. All properties except hydrogen:carbon ratios and the fraction of condensed aromatics are predicted within one experimental standard deviation.

A reaction model was assembled for the catalytic upgrading of coal resids. The key reaction families were identified and the most and least reactive resids for which kinetic data is being acquired (see above) will be used to optimize the rate constants. The model was coded and is being debugged and refined. The objective functions will consist of approximately eight terms. The only values used in the optimization will be the fraction of resid converted at each residence time for which experimental data were acquired. The model when completed will be able to incorporate additional analytical data to further define a unique set of rate constants. However, 
the eight terms currently being used to define the objective function should be sufficient to provide an adequate unique solution.

Delaware is drafting a Final Report and will complete and submit the completed structural/reaction model, documentation which describes the software, and an operating manual. 


\section{Section 4}

CONCLUSIONS

Conclusions are provided in the Executive Summary section of the report. 
Section 5

REFERENCES

1. HTI portion of Advanced Direct Liquefaction Concepts for PETC Generic Units, Quarterly Technical Progress Report for Period January through March 1997, U.S. Department of Energy, Report DOE/PC 91040-80, May 30,1997.

2. J. Mu (HTI), personal communication with G. A. Robbins (CONSOL).

3. LDP Associates portion of Advanced Direct Liquefaction Concepts for PETC Generic Units, Quarterly Technical Progress Report for Period January through March 1997, U.S. Department of Energy, Report DOE/PC 91040-80, May 30,1997.

4. Robbins, G. A.; Brandes, S. D.; Winschel, R. A.; Burke, F. P. "A Characterization and Evaluation of Coal Liquefaction Process Streams, Quarterly Technical Progress Report, July 1 through September 30, 1995", DOE/PC 93054-20, December 1995.

21 


\section{Section 6}

\section{LIST OF ABBREVIATIONS AND ACRONYMS}

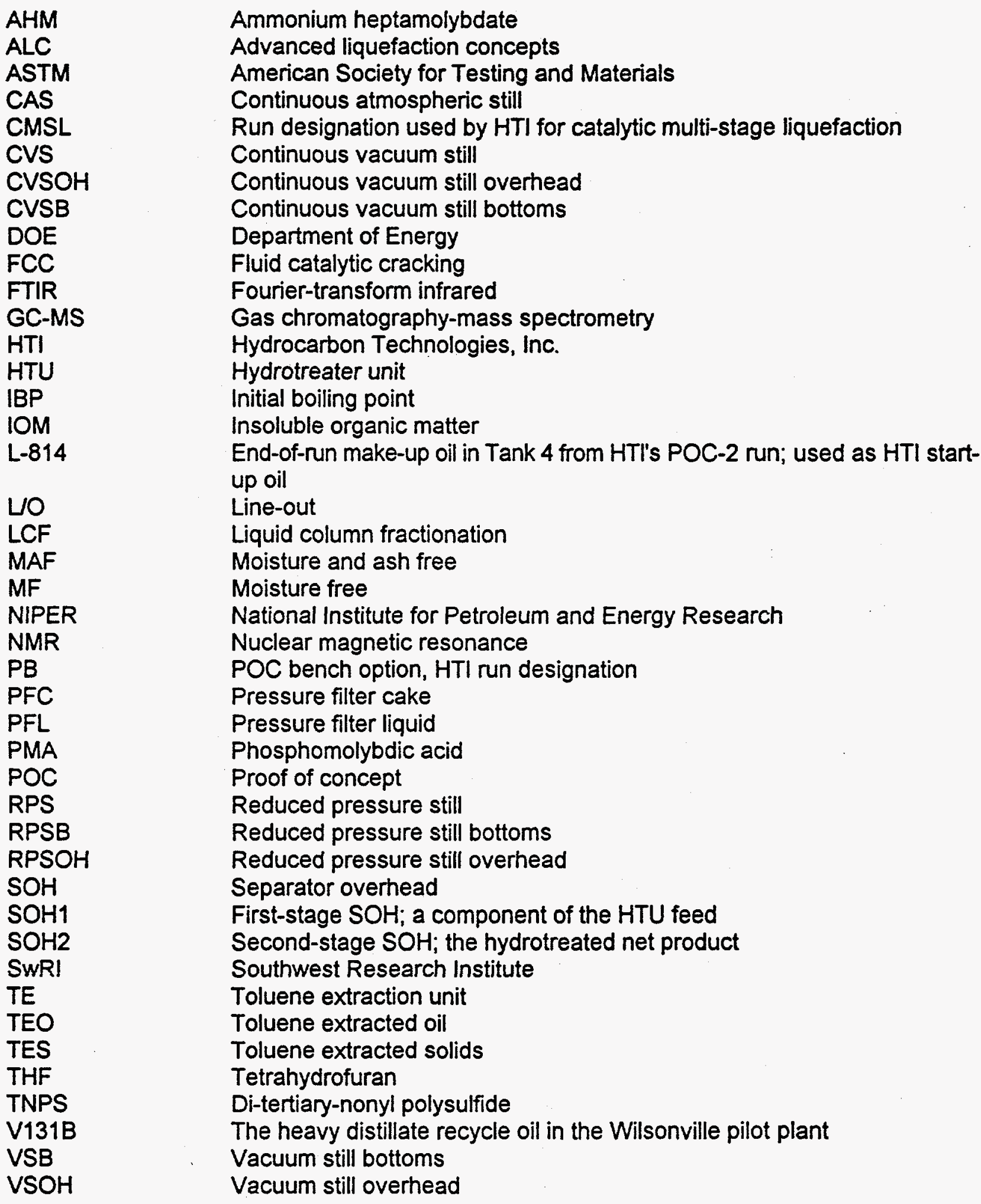


TABLE 1

RUN CONDITIONS AND YIELDS FOR HTI RUN ALC-2 (227-100)

\begin{tabular}{|c|c|c|c|c|}
\hline Date, 1996 & $12 / 8$ & $12 / 13$ & $12 / 18$ & $12 / 22$ \\
\hline Condition & 1 & 2 & 3 & 4 \\
\hline Period (work-up was last period in each condition) & $1-7$ & $8-12$ & $13-17$ & 18-21 \\
\hline $\begin{array}{l}\text { Net Normalized Yields, wt \% MAF Fresh Feed } \\
\mathrm{C}_{1} \text { Gases } \\
\mathrm{C}_{2} \text { Gases } \\
\mathrm{C}_{3} \text { Gases } \\
\mathrm{C}_{4} \text { Gases } \\
\mathrm{C}_{5} \text { Gases } \\
\mathrm{C}_{6} \text { \& } \mathrm{C}_{7} \text { Gases } \\
\mathrm{C}_{1}-\mathrm{C}_{3} \text { in Gases } \\
\mathrm{C}_{4}-\mathrm{C}_{7} \text { in Gases } \\
\text { IBP-177 }{ }^{\circ} \mathrm{C} \\
177-260^{\circ} \mathrm{C} \\
260-343^{\circ} \mathrm{C} \\
343-399^{\circ} \mathrm{C} \\
399-454^{\circ} \mathrm{C} \\
454-524^{\circ} \mathrm{C} \\
524^{\circ} \mathrm{C} \\
\text { Unconverted Coal } \\
\mathrm{Water}^{*} \\
\mathrm{CO}^{\mathrm{CO}_{2}} \\
\mathrm{NH}_{3} \\
\mathrm{H}_{2} \mathrm{~S}\end{array}$ & $\begin{array}{r}4.32 \\
4.11 \\
5.14 \\
2.86 \\
1.93 \\
2.86 \\
13.48 \\
7.65 \\
13.27 \\
16.34 \\
18.64 \\
3.73 \\
-1.44 \\
1.58 \\
9.32 \\
6.05 \\
13.36 \\
1.73 \\
5.74 \\
0.91 \\
0.13\end{array}$ & $\begin{array}{r}7.03 \\
3.97 \\
4.89 \\
2.67 \\
1.47 \\
2.63 \\
15.80 \\
6.77 \\
11.00 \\
11.16 \\
16.79 \\
0.76 \\
0.97 \\
2.98 \\
15.44 \\
4.80 \\
13.91 \\
1.47 \\
4.73 \\
0.69 \\
0.07\end{array}$ & $\begin{array}{r}6.01 \\
3.36 \\
4.21 \\
2.45 \\
1.57 \\
2.78 \\
13.50 \\
6.80 \\
11.94 \\
8.57 \\
16.27 \\
12.63 \\
6.37 \\
1.59 \\
4.77 \\
4.95 \\
15.78 \\
1.28 \\
3.47 \\
0.73 \\
0.31\end{array}$ & $\begin{array}{r}6.71 \\
3.83 \\
4.84 \\
2.51 \\
1.44 \\
2.48 \\
15.29 \\
6.43 \\
11.74 \\
11.56 \\
17.10 \\
3.45 \\
1.22 \\
2.33 \\
12.38 \\
4.29 \\
14.54 \\
0.98 \\
5.27 \\
0.72 \\
0.14\end{array}$ \\
\hline $\begin{array}{l}\text { Process Performance, wt } \% \text { MAF Fresh Feed } \\
\mathrm{H}_{2} \text { Consumption by Balance, } \mathrm{SO}_{3} \text { free } \\
\left.\text { Coal Conversion ( } \mathrm{SO}_{3} \text { Free) }\right)^{\star} \\
524^{+\circ} \mathrm{C} \text { Conversion } \\
\mathrm{C}_{4}-524^{\circ} \mathrm{C} \text { Distillates } \\
524^{+} \mathrm{C} \text { Resid Yield + Unconverted Coal }\end{array}$ & $\begin{array}{r}7.1 \\
93.9 \\
84.6 \\
59.8 \\
15.4\end{array}$ & $\begin{array}{r}7.0 \\
95.2 \\
79.8 \\
50.4 \\
20.2\end{array}$ & $\begin{array}{r}7.2 \\
95.1 \\
90.3 \\
64.2 \\
9.7\end{array}$ & $\begin{array}{r}6.9 \\
95.7 \\
83.3 \\
53.8 \\
16.7\end{array}$ \\
\hline $\begin{array}{l}\text { Catalysts (Impregnated on Coal) } \\
\text { Type } \\
\text { Concentration, mg/kg MF Coal }\end{array}$ & $\begin{array}{l}\text { AHMFe } \\
\text { Mo:100, } \\
\text { Fe: } 1610\end{array}$ & $\begin{array}{r}\text { AHM } \\
M o: 110\end{array}$ & $\begin{array}{c}\text { AHM/Ni } \\
\text { Mo:92, } \\
\text { Ni:51 }\end{array}$ & $\begin{array}{r}\text { PMA } \\
\text { Mo:116, } \\
P: 3\end{array}$ \\
\hline
\end{tabular}

-Coal conversion and unconverted coal are calculated based on ash balance.

\section{Common Conditions}

Temperatures, ${ }^{\circ} \mathrm{C}$ :

Space Velocity (each reactor):

Inlet Pressure:

$\mathrm{H}_{2}$ Rate:

Sulfiding Agents:
Pretreater, 300; Reactor 1, 440; Reactor 2, 450; In-Line Hydrotreater, 379; RPS, 316 (period 7 on); CVS, 427; Batch Vacuum Still (Condition 4 Only), 524 $640 \mathrm{~kg} \mathrm{MF}$ fresh feed $/ \mathrm{m}^{3}$ reactor volume

$17 \mathrm{MPa}$

20\% MF coal (3270 std L/h total, 1960:1100:210 Reactor 1:Reactor 2: Separators)

$\mathrm{H}_{2} \mathrm{~S}$ to Reactor $1,2 \%$ MF coal; TNPS to Reactor 2 equivalent to $\mathrm{H}_{2} \mathrm{~S}$ dose of $0.5 \%$ MF coal 
TABLE 2

CHARACTERISTICS OF CATALYST-TREATED COALS FED IN HTI RUN ALC-2

\begin{tabular}{|c|c|c|c|c|}
\hline Coal Sample and Run ALC-2 Condition Number & $\begin{array}{l}\text { L-902 } \\
\text { Cond. } 1\end{array}$ & $\begin{array}{l}\text { L-904 } \\
\text { Cond. } 2\end{array}$ & $\begin{array}{c}\text { L-906 } \\
\text { Cond. } 3\end{array}$ & $\begin{array}{l}\text { L-911 } \\
\text { Cond. } 4\end{array}$ \\
\hline $\begin{array}{l}\text { Moisture, wt \% As-Determined } \\
\text { Ash, wt \% MF, Including } \mathrm{SO}_{3} \\
\mathrm{SO}_{3} \text { Content Used to Correct Ash } \\
\text { Ash, wt \% MF, SO } \mathrm{SO}_{3} \text {-Free }\end{array}$ & $\begin{array}{r}12.68 \\
6.78 \\
18.95 \\
5.50\end{array}$ & $\begin{array}{r}13.07 \\
6.07 \\
17.11 \\
5.03\end{array}$ & $\begin{array}{r}10.46 \\
6.26 \\
16.73 \\
5.21\end{array}$ & $\begin{array}{r}11.16 \\
6.13 \\
16.86 \\
5.10\end{array}$ \\
\hline $\begin{array}{l}\text { Proximate, wt \% MF, SO3-Free Ash Basis } \\
\text { Volatile Matter } \\
\text { Fixed Carbon } \\
\text { Heating Value, Btu/b MAF, SO3-Free Ash Basis }\end{array}$ & $\begin{array}{r}46.58 \\
47.92 \\
12627\end{array}$ & $\begin{array}{r}46.58 \\
48.39 \\
12647\end{array}$ & $\begin{array}{r}46.68 \\
48.11 \\
12697\end{array}$ & $\begin{array}{r}47.07 \\
47.83 \\
12663\end{array}$ \\
\hline $\begin{array}{l}\text { Ultimate wt \% MF SO3-Free Ash Basis } \\
\mathrm{C} \\
\mathrm{H} \\
\mathrm{N} \\
\mathrm{S} \text {, Total } \\
\text { O (by Difference) }\end{array}$ & $\begin{array}{r}70.33 \\
4.75 \\
1.07 \\
0.58 \\
17.77\end{array}$ & $\begin{array}{r}70.71 \\
4.82 \\
1.03 \\
0.46 \\
17.95\end{array}$ & $\begin{array}{r}70.81 \\
4.78 \\
1.04 \\
0.48 \\
17.68\end{array}$ & $\begin{array}{r}70.05 \\
4.80 \\
1.04 \\
0.48 \\
18.53\end{array}$ \\
\hline $\begin{array}{l}\text { Maior Ash Elements. Oxide wt \% of } \mathrm{SO}_{3} \text {-Free Ash } \\
\mathrm{MoO}_{3} \\
\mathrm{NiO} \\
\mathrm{Na}_{2} \mathrm{O} \\
\mathrm{K}_{2} \mathrm{O} \\
\mathrm{CaO} \\
\mathrm{MgO} \\
\mathrm{Fe}_{2} \mathrm{O}_{3} \\
\mathrm{TiO}_{2} \\
\mathrm{P}_{2} \mathrm{O}_{5} \\
\mathrm{SiO}_{2} \\
\mathrm{Al}_{2} \mathrm{O}_{3} \\
\mathrm{Total}\end{array}$ & $\begin{array}{r}0.28 \\
0.01 \\
1.57 \\
0.47 \\
26.67 \\
5.43 \\
11.30 \\
1.44 \\
1.22 \\
34.62 \\
18.10 \\
101.11\end{array}$ & $\begin{array}{r}0.29 \\
0.01 \\
1.69 \\
0.54 \\
27.58 \\
5.60 \\
6.83 \\
1.42 \\
1.29 \\
35.84 \\
18.13 \\
99.23\end{array}$ & $\begin{array}{r}0.23 \\
0.11 \\
1.65 \\
0.54 \\
26.68 \\
5.58 \\
6.70 \\
1.50 \\
1.22 \\
36.17 \\
19.01 \\
99.41\end{array}$ & $\begin{array}{r}0.23 \\
0.01 \\
1.68 \\
0.49 \\
26.83 \\
5.65 \\
6.48 \\
1.50 \\
1.25 \\
35.37 \\
18.50 \\
98.02\end{array}$ \\
\hline $\begin{array}{l}\text { Sieve Size, Fraction wh \% of Total } \\
+48 \text { mesh } \\
48 \times 100 \text { mesh } \\
100 \times 200 \text { mesh } \\
200 \times 325 \text { mesh } \\
-325 \text { mesh }\end{array}$ & $\begin{array}{r}0.0 \\
7.8 \\
36.5 \\
19.2 \\
36.5\end{array}$ & $\begin{array}{r}0.0 \\
4.2 \\
42.2 \\
25.1 \\
28.5\end{array}$ & $\begin{array}{r}0.3 \\
7.8 \\
39.4 \\
20.4 \\
32.1\end{array}$ & $\begin{array}{r}0.4 \\
6.0 \\
47.5 \\
20.8 \\
25.3\end{array}$ \\
\hline $\begin{array}{l}\text { Particle Size Analysis of }-325 \text { mesh Fraction }(a, b) \\
m, \text { vol \% distribution } \\
d_{10} \\
d_{50} \\
d_{90}\end{array}$ & $\begin{array}{r}8.08 \\
28.53 \\
55.85\end{array}$ & $\begin{array}{r}8.14 \\
27.67 \\
50.12\end{array}$ & $\begin{array}{r}7.88 \\
28.87 \\
55.43\end{array}$ & $\begin{array}{r}8.97 \\
30.58 \\
54.47\end{array}$ \\
\hline $\begin{array}{l}\text { Mo wt \% of } \mathrm{SO}_{3} \text {-free ash } \\
\mathrm{Fe} \text { wt \% of } \mathrm{SO}_{3} \text {-free ash } \\
\text { ivi mg/kg of } \mathrm{SO}_{3} \text {-free ash }\end{array}$ & $\begin{array}{r}0.19 \\
7.90 \\
60\end{array}$ & $\begin{array}{r}0.19 \\
4.78 \\
75\end{array}$ & $\begin{array}{r}0.16 \\
4.69 \\
901\end{array}$ & $\begin{array}{r}0.16 \\
4.53 \\
59\end{array}$ \\
\hline $\begin{array}{l}\text { Mo wt \% of } \mathrm{SO}_{3} \text {-containing ash } \\
\mathrm{Fe} \mathrm{wt} \mathrm{\%} \mathrm{of} \mathrm{SO}_{3} \text {-containing ash } \\
\mathrm{Ni} \mathrm{mg} / \mathrm{kg} \text { of } \mathrm{SO}_{3} \text {-containing ash }\end{array}$ & $\begin{array}{r}0.15 \\
6.41 \\
49\end{array}$ & $\begin{array}{r}0.16 \\
3.96 \\
62\end{array}$ & $\begin{array}{r}0.13 \\
3.90 \\
750\end{array}$ & $\begin{array}{r}0.13 \\
3.77 \\
49\end{array}$ \\
\hline
\end{tabular}

(a) By Malvern laser scattering, $d_{x}=$ diameter representing xth percentile of volume distribution.

(b) For comparison, the Wilsonville Run 262 Black Thunder Mine feed coal gave $d_{10}=7 \mu \mathrm{m}, d_{50}=32 \mu \mathrm{m}$, and $d_{s 0}=72 \mu \mathrm{m}$ when measured by the same method. The Wilsonville feed coal was prepared at the same facility used by HTI, and the data given are for the entire feed, not a size subfraction as is reported for the Run ALC-2 feeds. 
TABLE 3

CONCENTRATIONS OF CATALYTIC METALS IN ASHY STREAMS FROM HTI RUN ALC-2

\begin{tabular}{|c|c|c|c|c|c|c|}
\hline \multirow[b]{2}{*}{ Sample } & \multirow[b]{2}{*}{$\begin{array}{c}\text { Cond. } \\
\text { No. }\end{array}$} & \multirow[b]{2}{*}{$\begin{array}{c}\text { Analyzed } \\
\text { by }\end{array}$} & \multirow[b]{2}{*}{ Basis } & \multicolumn{3}{|c|}{ Metal Concentration in Ash } \\
\hline & & & & $\begin{array}{l}\text { Mo, } \\
\text { wt } \%\end{array}$ & $\begin{array}{l}\text { Fe, } \\
\text { wt } \%\end{array}$ & $\begin{array}{c}\mathrm{Ni}, \\
\mathrm{mg} / \mathrm{kg}\end{array}$ \\
\hline Per. 1-7 CVSB(a) & 1 & $\mathrm{HTI}^{1}$ & Ash $\mathrm{SO}_{3}$ Uncorrected & 0.17 & 6.70 & NA \\
\hline Per. 8-12 CVSB & 2 & $\mathrm{HTI}^{1}$ & Ash $\mathrm{SO}_{3}$ Uncorrected & $0.15-0.19$ & $5.01-6.65$ & NA \\
\hline Per. 13-17 CVSB & 3 & $\mathrm{HTI}^{1}$ & $\mathrm{Ash} \mathrm{SO}_{3}$ Uncorrected & $0.17-0.20$ & $4.63-5.01$ & $184-631$ \\
\hline Per. 18-21 CVSB & 4 & $\mathrm{HTI^{1 }}$ & Ash $\mathrm{SO}_{3}$ Uncorrected & 0.16 & $4.59-4.75$ & 404-565 \\
\hline Coal L-902 & 1 & $\mathrm{HTI}^{1}$ & Ash $\mathrm{SO}_{3}$ Uncorrected & 0.16 & 5.25 & 49 \\
\hline Coal L-904 & 2 & $H T I^{1}$ & Ash $\mathrm{SO}_{3}$ Uncorrected & 0.18 & NA & NA \\
\hline Coal L-906 & 3 & $\mathrm{HTI}^{\mathrm{T}}$ & Ash $\mathrm{SO}_{3}$ Uncorrected & 0.15 & 3.18 & 829 \\
\hline Coal L-911 & 4 & $\mathrm{HTI}^{1}$ & Ash $\mathrm{SO}_{3}$ Uncorrected & 0.15 & 3.41 & 67 \\
\hline Coal L-902 & 1 & CONSOL & Ash $\mathrm{SO}_{3}$ Uncorrected & 0.15 & 6.41 & 49 \\
\hline Coal L-904 & 2 & CONSOL & Ash $\mathrm{SO}_{3}$ Uncorrected & 0.16 & 3.96 & 62 \\
\hline Coal L-906 & 3 & CONSOL & Ash $\mathrm{SO}_{3}$ Uncorrected & 0.13 & 3.90 & 750 \\
\hline Coal L-911 & 4 & CONSOL & $\mathrm{Ash} \mathrm{SO}_{3}$ Uncorrected & 0.13 & 3.77 & 49 \\
\hline Coal L-902 & 1 & CONSOL & Ash $\mathrm{SO}_{3}$-Free & 0.19 & 7.90 & 60 \\
\hline \multicolumn{7}{|c|}{ TES Samples, Compare with Coal L-902 } \\
\hline Per. 2AB TES & 1 & CONSOL & $\begin{array}{l}\text { Norm. } \mathrm{SO}_{3} \\
\text { and } \mathrm{K}_{2} \mathrm{O} \text { - Free }\end{array}$ & 0.15 & 7.41 & 258 \\
\hline Per. 3B TES & 1 & CONSOL & $\begin{array}{l}\text { Norm. } \mathrm{SO}_{3}^{-} \\
\text {and } \mathrm{K}_{2} \mathrm{O} \text { - Free }\end{array}$ & 0.22 & 10.04 & 331 \\
\hline Per. 5AB TES & 1 & CONSOL & $\begin{array}{l}\text { Norm. } \mathrm{SO}_{3-} \\
\text { and } \mathrm{K}_{2} \mathrm{O} \text { - Free }\end{array}$ & 0.07 & 4.63 & 125 \\
\hline Per. 6 TES & 1 & CONSOL & $\begin{array}{l}\text { Norm. } \mathrm{SO}_{3^{-}} \\
\text {and } \mathrm{K}_{2} \mathrm{O} \text { - Free }\end{array}$ & 0.21 & 8.69 & 135 \\
\hline
\end{tabular}

(a) HTI analyzed only the period 7 sample.

NA $=$ not available 
TABLE 4

CHARACTERISTICS OF TOLUENE-EXTRACTED SOLIDS FROM CONDITION 1 OF HTI RUN ALC-2

\begin{tabular}{|c|c|c|c|c|}
\hline Run ALC-2 Period Number & Per. 2AB & Per. 3B & Per. 5AB & Per. 6 \\
\hline $\begin{array}{l}\text { Toluene, wt \% As-Determined (By Evaporation) } \\
\text { Ash, wt \% As-Determined, Including } \mathrm{SO}_{3} \text { (a) } \\
\text { Unaccounted, wt \% of } \mathrm{SO}_{3} \text {-Containing Ash (b) } \\
\text { Ash, wt \% As-Determined, Calculated, } \mathrm{SO}_{3} \text { - and } \\
\mathrm{K}_{2} \mathrm{O} \text {-Free (c) }\end{array}$ & $\begin{array}{r}10.4 \\
7.95 \\
21.94 \\
6.21\end{array}$ & $\begin{array}{r}2.8 \\
25.03 \\
16.80 \\
20.82\end{array}$ & $\begin{array}{r}15.0 \\
10.04 \\
20.34 \\
8.00\end{array}$ & $\begin{array}{r}25.7 \\
10.45 \\
21.80 \\
8.17\end{array}$ \\
\hline $\begin{array}{l}\text { Major and Minor Ash Elements. Oxide wt \% of } \mathrm{SO}_{3}= \\
\text { and } \mathrm{K}_{2} \mathrm{O} \text {-Free Ash (c) } \\
\mathrm{MoO}_{3} \\
\mathrm{NiO} \\
\mathrm{Na} \mathrm{O}_{2} \mathrm{O} \\
\mathrm{CaO} \\
\mathrm{MgO} \\
\mathrm{Fe}_{2} \mathrm{O}_{3} \\
\mathrm{TiO}_{2} \\
\mathrm{P}_{2} \mathrm{O}_{5} \\
\mathrm{SiO}_{2} \\
\mathrm{Al}_{2} \mathrm{O}_{3}\end{array}$ & $\begin{array}{r}0.22 \\
0.03 \\
2.39 \\
33.59 \\
7.21 \\
10.60 \\
2.15 \\
1.48 \\
23.44 \\
18.88\end{array}$ & $\begin{array}{r}0.34 \\
0.04 \\
2.14 \\
30.44 \\
6.29 \\
14.35 \\
1.84 \\
1.43 \\
25.17 \\
17.97\end{array}$ & $\begin{array}{r}0.10 \\
0.02 \\
2.19 \\
33.59 \\
7.05 \\
6.61 \\
2.09 \\
1.43 \\
27.55 \\
19.37\end{array}$ & $\begin{array}{r}0.32 \\
0.02 \\
2.14 \\
36.64 \\
7.71 \\
12.42 \\
2.25 \\
1.68 \\
18.32 \\
18.50\end{array}$ \\
\hline $\begin{array}{l}\text { Metal Concentration in } \mathrm{SO}_{3} \text { - and } \mathrm{K}_{2} \mathrm{O} \text {-Free Ash } \\
\mathrm{Mo}, \text { wt } \% \\
\mathrm{Fe}, \mathrm{wt} \% \\
\mathrm{Ni}, \mathrm{mg} / \mathrm{kg}\end{array}$ & $\begin{array}{r}0.15 \\
7.41 \\
258\end{array}$ & $\begin{array}{r}0.22 \\
10.04 \\
331\end{array}$ & $\begin{array}{r}0.07 \\
4.63 \\
125\end{array}$ & $\begin{array}{r}0.21 \\
8.69 \\
135\end{array}$ \\
\hline
\end{tabular}

(a) ASTM ash of whole sample.

(b) Weight percent of ASTM ash that was not accounted for by summing the contributions of oxides determined $\left(\mathrm{SO}_{3}\right.$ and $\mathrm{K}_{2} \mathrm{O}$ not determined).

(c) Element concentrations were determined on an as-determined basis by digestion of the whole sample and analysis via a procedure that did not include sulfur and potassium (the digestion procedure adds potassium). Normalized $\mathrm{SO}_{3}$ - and $\mathrm{K}_{2} \mathrm{O}$-free ash content was obtained by summing all of the elemental concentrations in their oxide forms. 
TABLE 5

COMPONENT DISTRIBUTION OF TES AND TEO SAMPLES HTI RUN ALC-2 (227-100)

\begin{tabular}{||c|c|c|c|c|c|c|c||}
\hline & & & \multicolumn{3}{|c|}{$w$ \% Toluene-Free Sample } & \\
\hline Sample & Period & Condition & $\begin{array}{c}\text { Toluene, } w \text { \% } \% \\
\text { of Whole } \\
\text { Sample }\end{array}$ & THF-Solubles & IOM & Ash & $\begin{array}{c}\text { Toluene, } w \text { of } \% \\
\text { Toluene-Free } \\
\text { Sample }\end{array}$ \\
\hline & & & & & & & \\
\hline TES & $2 \mathrm{AB}$ & 1 & 10.4 & 81.7 & 10.0 & 8.3 & 11.6 \\
\hline TES & $3 \mathrm{~B}(\mathrm{a})$ & 1 & 2.8 & 32.3 & 42.4 & 25.3 & 2.9 \\
\hline TES & $3 \mathrm{~B}(\mathrm{~b})$ & 1 & 81.4 & 28.8 & 47.3 & 23.9 & 437.6 \\
\hline TES & $5 \mathrm{AB}$ & 1 & 15.0 & 78.6 & 9.8 & 11.6 & 17.6 \\
\hline TES & 6 & 1 & 25.7 & 74.2 & 11.7 & 14.1 & 34.6 \\
\hline TES & $7 \mathrm{~B}$ & 1 & 7.4 & 74.9 & 10.4 & 14.7 & 8.0 \\
\hline TES & $9 \mathrm{~A} / \mathrm{B}$ & 2 & 70.0 & 21.2 & 38.6 & 40.2 & 233.3 \\
\hline TES & 12 & 2 & 43.0 & 42.7 & 25.2 & 32.0 & 75.3 \\
\hline TES & 13 & 2 & 47.1 & 48.9 & 24.0 & 27.1 & 89.0 \\
\hline & & & & & & & \\
\hline TEO & 7 & 1 & 21.3 & 85.6 & 7.0 & 7.4 & 27.1 \\
\hline TEO & 12 & 2 & 90.8 & 96.7 & 2.2 & 1.1 & 987.0 \\
\hline \hline
\end{tabular}

Toluene was determined by weight loss (to constant weight) in a vacuum oven at $60^{\circ} \mathrm{C}$ and full vacuum (pressure ca. $5 \mathrm{~mm} \mathrm{Hg}$, atmospheric equivalent was less than $190^{\circ} \mathrm{C}$ ) for up to several days. Some $650^{\circ} \mathrm{F}^{-}$oil may be reported as toluene by this method (cf. text).

(a) First sample received from HTI from this period; also analyzed for proton distribution and phenolic - OH concentration (reported in other tables).

(b) Second sample received from $\mathrm{HTI}$ from this period. 
TABLE 6

MISCELLANEOUS ANALYSES OF CVSB AND 0-6 BOTTOMS SAMPLES HTI RUN ALC-2 (227-100)

\begin{tabular}{|c|c|c|c|c|}
\hline Condition & 1 & 2 & 3 & 4 \\
\hline Period & 7B & 12B & 17B & 21B \\
\hline \multicolumn{5}{|c|}{$\%$ MAF Coal Conversion in Microautoclave Donor Solvent Assay(a) } \\
\hline Q-6 Btms $454^{\circ} \mathrm{C}-$ Distillate & 82.9 & 85.3 & 84.9 & 82.8 \\
\hline \multicolumn{5}{|c|}{ Component, wt \% of Whole O-6 Btms Sample } \\
\hline $454^{\circ} \mathrm{C}$-Distillate & 42.7 & 38.0 & 38.2 & 42.3 \\
\hline $454^{\circ} \mathrm{C}^{+}$Resid THF Solubles & 36.8 & 37.9 & 37.9 & 35.2 \\
\hline IOM & 6.6 & 8.2 & 8.9 & 7.3 \\
\hline Ash & 9.2 & 12.0 & 11.7 & 10.6 \\
\hline & & & & \\
\hline Period & 7B & 12 & 17 & 21 \\
\hline \multicolumn{5}{|c|}{ Component, wt \% of Whole CVSB Sample } \\
\hline Ash & 13.6 & 19.4 & 16.4 & 16.7 \\
\hline$O M$ & 10.0 & 14.2 & 12.8 & 11.0 \\
\hline THF-Solubles & 76.4 & 66.4 & 70.8 & 72.3 \\
\hline \multicolumn{5}{|c|}{ Component, wt $\%$ of CVSB THF Solubles } \\
\hline wt \% Oils & 67.2 & 69.5 & 68.9 & 78.2 \\
\hline ut \% Asph. & 22.4 & 21.1 & 21.8 & 14.1 \\
\hline ut \% Preasph & 10.4 & 9.4 & 9.3 & 7.7 \\
\hline
\end{tabular}

(a) Standard Indiana 5 test coal, modified-equilibrium test conditions: $9 \mathrm{~g}$ solvent, $6 \mathrm{~g}$ coal, $399^{\circ} \mathrm{C}, 30 \mathrm{~min}$. 
TABLE 7

PROTON DISTRIBUTION OF SAMPLES BY NMR HTI RUN ALC-2 (227-100)

\begin{tabular}{|c|c|c|c|c|c|c|c|c|c|}
\hline \multirow[b]{2}{*}{ Sample } & \multirow[b]{2}{*}{ Period } & \multirow[b]{2}{*}{$\begin{array}{l}\text { Condi- } \\
\text { tion }\end{array}$} & \multicolumn{7}{|c|}{ Proton Distribution, $\%$} \\
\hline & & & $\begin{array}{l}\text { Cond. } \\
\text { Arom }\end{array}$ & $\begin{array}{c}\text { Uncond. } \\
\text { Arom }\end{array}$ & $\begin{array}{l}\text { Cyclic } \\
\text { Alpha } \\
\end{array}$ & $\begin{array}{l}\text { Alkyl } \\
\text { Alpha }\end{array}$ & $\begin{array}{c}\text { Cyclic } \\
\text { Beta }\end{array}$ & $\begin{array}{l}\text { Alkyl } \\
\text { Beta }\end{array}$ & Gamma \\
\hline \multicolumn{10}{|l|}{ Whole Samples } \\
\hline $\begin{array}{l}\text { L-814 Start-Up Oil } \\
1 / 10 / 97 \text { (a) }\end{array}$ & & SN & 9.7 & 5.1 & 10.7 & 8.8 & 13.9 & 32.3 & 19.5 \\
\hline CVSB & $7 B$ & 1 & 24.3 & 11.5 & 18.0 & 10.6 & 11.5 & 15.9 & 8.2 \\
\hline CVSB & $12 \mathrm{~B}$ & 2 & 25.8 & 11.9 & 19.7 & 10.1 & 12.0 & 13.3 & 7.2 \\
\hline CVSB & $17 \mathrm{~B}$ & 3 & 25.0 & 11.6 & 18.5 & 9.9 & 12.1 & 14.7 & 8.2 \\
\hline CVSB & $21 \mathrm{~B}$ & 4 & 27.1 & 9.0 & 19.7 & 9.7 & 11.5 & 14.9 & 7.9 \\
\hline O-6 Btms & $7 B$ & 1 & 18.8 & 12.2 & 15.4 & 11.4 & 11.9 & 19.2 & 11.1 \\
\hline $0-6 \mathrm{Btms}$ & 12B & 2 & 19.7 & 10.9 & 17.7 & 11.2 & 12.5 & 18.1 & 9.9 \\
\hline $0-6$ Btms & $17 \mathrm{~B}$ & 3 & 19.0 & 12.4 & 16.3 & 10.8 & 12.6 & 18.6 & 10.3 \\
\hline O-6 Btms & $21 B$ & 4 & 19.6 & 12.4 & 16.2 & 11.5 & 11.9 & 18.7 & 9.7 \\
\hline RPSOH & $7 \mathrm{~B}$ & 1 & 8.3 & 12.1 & 13.3 & 13.9 & 14.8 & 24.3 & 13.3 \\
\hline RPSOH & $12 \mathrm{~B}$ & 2 & 7.3 & 13.9 & 15.1 & 15.1 & 14.6 & 22.4 & 11.6 \\
\hline RPSOH & $17 \mathrm{~B}$ & 3 & 10.2 & 11.9 & 15.1 & 13.5 & 15.1 & 22.6 & 11.6 \\
\hline RPSOH & 21B & 4 & 10.4 & 12.0 & 14.3 & 13.3 & 14.4 & 23.2 & 12.2 \\
\hline RPSB & $7 B$ & 1 & 16.5 & 8.8 & 14.9 & 11.1 & 13.1 & 23.8 & 11.9 \\
\hline RPSB & $12 B$ & 2 & 18.3 & 9.0 & 16.5 & 10.8 & 12.5 & 22.5 & 10.4 \\
\hline RPSB & $16 \mathrm{~B}$ & 3 & 17.0 & 9.4 & 15.7 & 10.6 & 12.2 & 24.0 & 11.2 \\
\hline RPSB & $17 \mathrm{~B}$ & 3 & 16.2 & 9.6 & 15.7 & 10.7 & 12.8 & 23.8 & 11.2 \\
\hline RPSB & $21 B$ & 4 & 18.0 & 9.1 & 16.1 & 10.7 & 12.9 & 22.4 & 10.7 \\
\hline TES (b) & $2 A B$ & 1 & 25.3 & 12.3 & 16.3 & 11.2 & 10.8 & 15.9 & 8.2 \\
\hline TES (b) & $3 B$ & 1 & 31.0 & 14.6 & 15.0 & 12.3 & 8.9 & 10.3 & 8.0 \\
\hline TES (b) & $5 \mathrm{~A} / \mathrm{B}$ & 1 & 24.1 & 12.3 & 16.9 & 10.9 & 11.8 & 15.4 & 8.6 \\
\hline TES (b) & 6 & 1 & 24.5 & 11.5 & 18.3 & 10.7 & 11.1 & 15.0 & 9.0 \\
\hline TES & $7 B$ & 1 & 25.9 & 9.8 & 19.4 & 10.6 & 11.9 & 15.3 & 7.2 \\
\hline TES & 9AB & 2 & 22.4 & 5.1 & 15.9 & 7.2 & 12.8 & 17.3 & 19.2 \\
\hline TES (b) & $12 \mathrm{AB}$ & 2 & 32.3 & 7.3 & 19.3 & 9.7 & 11.8 & 12.1 & 7.5 \\
\hline TES & 13AVB & 3 & 26.3 & 10.8 & 19.8 & 10.1 & 12.6 & 13.5 & 7.0 \\
\hline TEO & 7 & 1 & 22.8 & 8.9 & 15.7 & 10.2 & 12.2 & 19.3 & 10.9 \\
\hline TEO & 12 & 2 & 25.8 & 9.7 & 19.0 & 10.3 & 12.2 & 14.5 & 8.6 \\
\hline Stage $1 \mathrm{SOH}$ & 18 & 1 & 4.4 & 5.1 & 6.3 & 8.9 & 15.0 & 35.4 & 24.8 \\
\hline
\end{tabular}




\begin{tabular}{|c|c|c|c|c|c|c|c|c|c|}
\hline \multirow[b]{2}{*}{ Sample } & \multirow[b]{2}{*}{ Period } & \multirow[b]{2}{*}{$\begin{array}{c}\text { Condi- } \\
\text { tion }\end{array}$} & \multicolumn{7}{|c|}{ Proton Distribution, $\%$} \\
\hline & & & \begin{tabular}{|l|} 
Cond. \\
Arom
\end{tabular} & $\begin{array}{c}\text { Uncond. } \\
\text { Arom }\end{array}$ & $\begin{array}{l}\text { Cyclic } \\
\text { Alpha }\end{array}$ & $\begin{array}{l}\text { Alkyl } \\
\text { Alpha }\end{array}$ & $\begin{array}{c}\text { Cyclic } \\
\text { Beta }\end{array}$ & $\begin{array}{l}\text { Alkyl } \\
\text { Beta }\end{array}$ & Gamma \\
\hline Stage $1 \mathrm{SOH}$ & 78 & 1 & 6.0 & 10.3 & 9.6 & 12.0 & 16.5 & 27.6 & 18.0 \\
\hline Stage $1 \mathrm{SOH}$ & $12 B$ & 2 & 5.8 & 11.2 & 9.7 & 12.1 & 16.4 & 27.8 & 16.9 \\
\hline Stage $1 \mathrm{SOH}$ & $13 \mathrm{~A}$ & $2-3$ & 6.2 & 10.7 & 10.1 & 12.2 & 17.4 & 27.0 & 16.5 \\
\hline Stage $1 \mathrm{SOH}$ & $17 A$ & 3 & 5.3 & 10.8 & 8.9 & 11.7 & 17.1 & 27.8 & 18.5 \\
\hline Stage $1 \mathrm{SOH}$ & $21 \mathrm{~A}$ & 4 & 5.7 & 9.8 & 9.2 & 11.2 & 16.8 & 28.7 & 18.5 \\
\hline Stage $2 \mathrm{SOH}$ & $7 A$ & 1 & 0.5 & 2.5 & 3.1 & 4.0 & 23.0 & 36.4 & 30.5 \\
\hline Stage $2 \mathrm{SOH}$ & $12 \mathrm{~A}$ & 2 & 1.1 & 3.6 & 5.5 & 5.7 & 23.8 & 34.0 & 26.4 \\
\hline Stage $2 \mathrm{SOH}$ & $17 \mathrm{~A}$ & 3 & 0.9 & 3.2 & 4.7 & 5.3 & 23.6 & 35.4 & 26.9 \\
\hline Stage $2 \mathrm{SOH}$ & $17 B$ & 3 & 1.3 & 3.2 & 5.8 & 5.8 & 24.0 & 34.5 & 25.3 \\
\hline Stage $2 \mathrm{SOH}$ & $21 \mathrm{~A}$ & 4 & 0.7 & 2.0 & 2.7 & 4.1 & 24.5 & 35.7 & 30.3 \\
\hline Stage $2 \mathrm{SOH}$ & $21 B$ & 4 & 0.7 & 2.2 & 2.2 & 3.6 & 23.2 & 36.5 & 31.6 \\
\hline $454^{\circ} \mathrm{C}^{-}$Distilla & & & & & & & & & \\
\hline O-6 Btms & $7 \mathrm{~B}$ & 1 & 15.9 & 9.2 & 15.1 & 11.7 & 12.9 & 23.4 & 11.7 \\
\hline O-6 Btms & $12 B$ & 2 & 15.2 & 10.3 & 16.3 & 11.9 & 13.6 & 22.4 & 10.3 \\
\hline O-6 Btms & $17 \mathrm{~B}$ & 3 & 13.9 & 11.2 & 15.7 & 12.1 & 13.4 & 22.3 & 11.4 \\
\hline O-6 Btms & 21B & 4 & 17.0 & 10.3 & 15.8 & 11.5 & 12.4 & 22.7 & 10.3 \\
\hline $454^{\circ} \mathrm{C}^{+}$Resid & & & & & & & & & \\
\hline O-6 Btms & $7 B$ & 1 & 27.8 & 9.8 & 21.2 & 10.4 & 12.1 & 12.3 & 6.3 \\
\hline O-6 Btms & $12 B$ & 2 & 27.4 & 9.4 & 20.5 & 10.0 & 11.9 & 13.3 & 7.5 \\
\hline 0.6 Btms & $17 \mathrm{~B}$ & 3 & 27.6 & 9.5 & 20.8 & 9.7 & 12.4 & 13.2 & 6.9 \\
\hline $0-6 \mathrm{Btms}$ & $21 B$ & 4 & 29.3 & 11.4 & 19.9 & 10.2 & 11.4 & 11.8 & 5.9 \\
\hline
\end{tabular}

(a) The L-814 tank is the source of start-up oil, although the date (1/10/97) of this sample suggests that it was obtained after the run. This sample appeared to be much lower in viscosity than a 1995 sample from the same tank. See text and a later table relating to comparative L-814 data over several years.

(b) These samples were dissolved in $99.96 \% \mathrm{~d}_{5}$-pyridine, filtered, and integrated electronically. All other samples were dissolved in $99.96 \% d-\mathrm{CHCl}_{3}$ and integrated electronically. 
TABLE 8

\section{PHENOLIC -OH CONCENTRATION OF SAMPLES DETERMINED BY FTIR HTI RUN ALC-2 (227-100)}

\begin{tabular}{|c|c|c|c|c|c|c|c|c|c|}
\hline \multirow[b]{2}{*}{ Sample } & \multirow[b]{2}{*}{ Condition } & \multirow[b]{2}{*}{ Period } & \multicolumn{2}{|c|}{ Phenolic -OH } & \multirow[b]{2}{*}{ Sample } & \multirow[b]{2}{*}{ Condition } & \multirow[b]{2}{*}{ Period } & \multicolumn{2}{|c|}{ Phenolic - $\mathrm{OH}$} \\
\hline & & & $\begin{array}{l}\text { Conc., } \\
\text { meg/g }\end{array}$ & $\begin{array}{l}\text { Peak } \\
\text { Pos., } \\
\mathrm{cm}^{-1}\end{array}$ & & & & $\begin{array}{l}\text { Conc., } \\
\text { meq/g }\end{array}$ & $\begin{array}{l}\text { Peak } \\
\text { Pos., } \\
\text { cm }^{-1}\end{array}$ \\
\hline \multicolumn{5}{|c|}{ Whole Sample THF Solubles } & \multicolumn{5}{|c|}{ Whole Samples } \\
\hline CVSB & 1 & $7 B$ & 0.96 & 3295 & Stage $1 \mathrm{SOH}$ & 1 & $1 B$ & 0.65 & 3311 \\
\hline CVSB & 2 & $12 B$ & 0.91 & 3295 & Stage $1 \mathrm{SOH}$ & 1 & 7B & 1.70 & 3309 \\
\hline CVSB & 3 & 178 & 0.95 & 3295 & Stage $1 \mathrm{SOH}$ & 2 & $12 \mathrm{~B}$ & 1.87 & 3309 \\
\hline \multirow[t]{2}{*}{ CVSB } & 4 & 218 & 0.90 & 3293 & Stage $1 \mathrm{SOH}$ & 2 & $13 \mathrm{~A}$ & 1.90 & 3309 \\
\hline & & & & & Stage $1 \mathrm{SOH}$ & 3 & $17 \mathrm{~A}$ & 1.87 & 3309 \\
\hline TES & 1 & $2 A \sqrt{B}$ & 0.83 & 3292 & Stage $1 \mathrm{SOH}$ & 4 & $21 \mathrm{~A}$ & 1.61 & 3309 \\
\hline TES & 1 & $3 B$ & 1.11 & 3292 & & & & & \\
\hline TES & 1 & $5 A / B$ & 0.76 & 3295 & Stage $2 \mathrm{SOH}$ & 1 & $7 A$ & ND & \\
\hline TES & 1 & 6 & 0.88 & 3294 & Stage $2 \mathrm{SOH}$ & 2 & $12 A$ & ND & \\
\hline TES & 1 & $7 B$ & 0.77 & 3292 & Stage $2 \mathrm{SOH}$ & 3 & $17 \mathrm{~A}$ & ND & \\
\hline TES & 2 & $9 A \sqrt{ }$ & 0.98 & 3286 & Stage $2 \mathrm{SOH}$ & 3 & $17 \mathrm{~B}$ & ND & \\
\hline TES & 2 & $12 A \sqrt{B}$ & 1.06 & 3292 & Stage $2 \mathrm{SOH}$ & 4 & $21 \mathrm{~A}$ & ND & \\
\hline \multirow[t]{2}{*}{ TES } & 2 & $13 A \mathrm{~B}$ & 1.00 & 3292 & Stage $2 \mathrm{SOH}$ & 4 & $21 \mathrm{~B}$ & ND & \\
\hline & & & & & & & & & \\
\hline TEO & 1 & 7 & 0.70 & 3296 & RPSOH & 1 & $7 \mathrm{~B}$ & 1.78 & 3311 \\
\hline \multirow[t]{2}{*}{ TEO } & 2 & 12 & 0.68 & 3296 & RPSOH & 2 & $12 B$ & 1.49 & 3310 \\
\hline & & & & & RPSOH & 3 & $17 \mathrm{~B}$ & 1.89 & 3310 \\
\hline \multicolumn{5}{|c|}{$454^{\circ} \mathrm{C}-$ Distillates } & RPSOH & 4 & 21B & 1.60 & 3311 \\
\hline O-6 Bottoms & 1 & 78 & 1.02 & 3307 & & & & & \\
\hline O-6 Bottoms & 2 & $12 B$ & 1.12 & 3307 & RPSB & 1 & 7B & 0.81 & 3304 \\
\hline O-6 Bottoms & 3 & $17 B$ & 1.22 & 3307 & RPSB & 2 & $12 B$ & 0.94 & 3302 \\
\hline \multirow[t]{2}{*}{ O-6 Bottoms } & 4 & $21 B$ & 1.24 & 3307 & RPSB & 3 & $16 \mathrm{~B}$ & 0.85 & 3302 \\
\hline & & & & & RPSB & 3 & 17B & 0.91 & 3303 \\
\hline \multicolumn{5}{|c|}{$454^{\circ} \mathrm{C}^{+}$Resid THF Solubles } & RPSB & 4 & $21 B$ & 0.85 & 3302 \\
\hline O-6 Bottoms & 1 & $7 B$ & 0.98 & 3294 & & & & & \\
\hline O-6 Bottoms & 2 & $12 B$ & 0.96 & 3294 & & & & & \\
\hline O-6 Bottoms & 3 & $17 \mathrm{~B}$ & 0.93 & 3294 & & & & & \\
\hline O-6 Bottoms & 4 & 21B & 1.00 & 3294 & & & & & \\
\hline
\end{tabular}

ND $=$ None Detected 
TABLE 9

\section{PROTON DISTRIBUTIONS OF HTI L-814 START-UP OIL OVER TIME}

\begin{tabular}{|c|c|c|c|c|c|c|c|}
\hline & \multicolumn{7}{|c|}{ Proton Distribution, $\%$} \\
\hline $\begin{array}{l}\text { L-814 Sample } \\
\text { Origin }\end{array}$ & $\begin{array}{l}\text { Cond. } \\
\text { Arom }\end{array}$ & $\begin{array}{l}\text { Uncond. } \\
\text { Arom }\end{array}$ & $\begin{array}{l}\text { Cyclic } \\
\text { Alpha }\end{array}$ & $\begin{array}{l}\text { Alkyl } \\
\text { Alpha }\end{array}$ & $\begin{array}{l}\text { Cyclic } \\
\text { Beta }\end{array}$ & $\begin{array}{l}\text { Alkyl } \\
\text { Beta }\end{array}$ & Gamma \\
\hline \multicolumn{8}{|c|}{ Recent Sample, ca. 6/30/97 (ca. 3 mo. after Run PB-07) } \\
\hline Unspecified & 5.0 & 3.0 & 11.0 & 7.2 & 17.2 & 38.3 & 18.4 \\
\hline \multicolumn{8}{|c|}{ Run ALC-2 (227-100), 1/10/97 (a) } \\
\hline Per. S/U & 9.7 & 5.1 & 10.7 & 8.8 & 13.9 & 32.3 & 19.5 \\
\hline \multicolumn{8}{|c|}{ Run ALC-1 (227-94), Spring 1996} \\
\hline Per. 5 & 14.0 & 6.6 & 13.3 & 12.5 & 13.5 & 24.0 & 16.2 \\
\hline \multicolumn{8}{|c|}{ Run CMSL-9, (227-87), run ca. 5/11/95 } \\
\hline Per. 19 & 15.3 & 5.1 & 15.3 & 12.3 & 13.4 & 24.7 & 13.9 \\
\hline Per. 24 & 14.8 & 5.9 & 13.6 & 12.5 & 12.7 & 25.4 & 15.1 \\
\hline Per. 29 & 14.1 & 6.5 & 13.4 & 13.3 & 12.5 & 24.9 & 15.2 \\
\hline Per. 34 & 16.9 & 6.4 & 13.6 & 14.1 & 11.4 & 23.8 & 13.8 \\
\hline Per. 38 & 15.0 & 5.7 & 14.7 & 12.1 & 13.1 & 25.1 & 14.3 \\
\hline Per. 41 & 14.5 & 5.9 & 14.2 & 12.1 & 12.8 & 25.0 & 15.6 \\
\hline \multicolumn{8}{|c|}{ Run POC-2, Tank 4, ca. 6/94 } \\
\hline $\begin{array}{l}\text { Per. 18, Cond. } \\
\text { UO-1 }\end{array}$ & 15.9 & 7.1 & 14.6 & 14.6 & 12.2 & 21.4 & 14.1 \\
\hline
\end{tabular}

(a) Sample date suggest that the sample may have been taken after the run was completed. Sample received by CONSOL appeared to be low in viscosity. 
TABLE 10

ELEMENTAL ANALYSES OF RESIDUES

\begin{tabular}{|c|c|c|c|c|}
\hline & $\begin{array}{c}\text { Resid } \\
\text { W } 260 \\
\text { V131B(a) }\end{array}$ & $\begin{array}{c}\text { Residue Of Room } \\
\text { Temperature } \\
\text { Tetralin Washing }\end{array}$ & $\begin{array}{c}\text { Residue of } \\
\text { Tetralin Soxhlet } \\
\text { Extraction }\end{array}$ & $\begin{array}{c}\text { Unconverted } \\
454^{\circ} \mathrm{C}^{+} \\
\left(850^{\circ} \mathrm{F}^{+}\right) \text {Residue }\end{array}$ \\
\hline \multicolumn{5}{|c|}{ Ultimate, wt \% } \\
\hline C & 82.16 & 65.01 & 52.87 & 44.34 \\
\hline$H$ & 5.93 & 3.21 & 1.83 & 1.56 \\
\hline $\mathbf{N}$ & 0.90 & 1.21 & 1.01 & 0.69 \\
\hline$S_{t x t}$ & 1.13 & 2.67 & 3.78 & 5.81 \\
\hline Ash & 8.57 & 24.53 & 37.94 & 46.78 \\
\hline$O$ (by diff) & 1.31 & 3.37 & 2.57 & 0.82 \\
\hline $\mathrm{H} / \mathrm{C}$ & 0.87 & 0.59 & 0.42 & 0.42 \\
\hline
\end{tabular}

(a) W 260 V131B = Wilsonville Run 260, Sampling location V131B (recycle stream) 


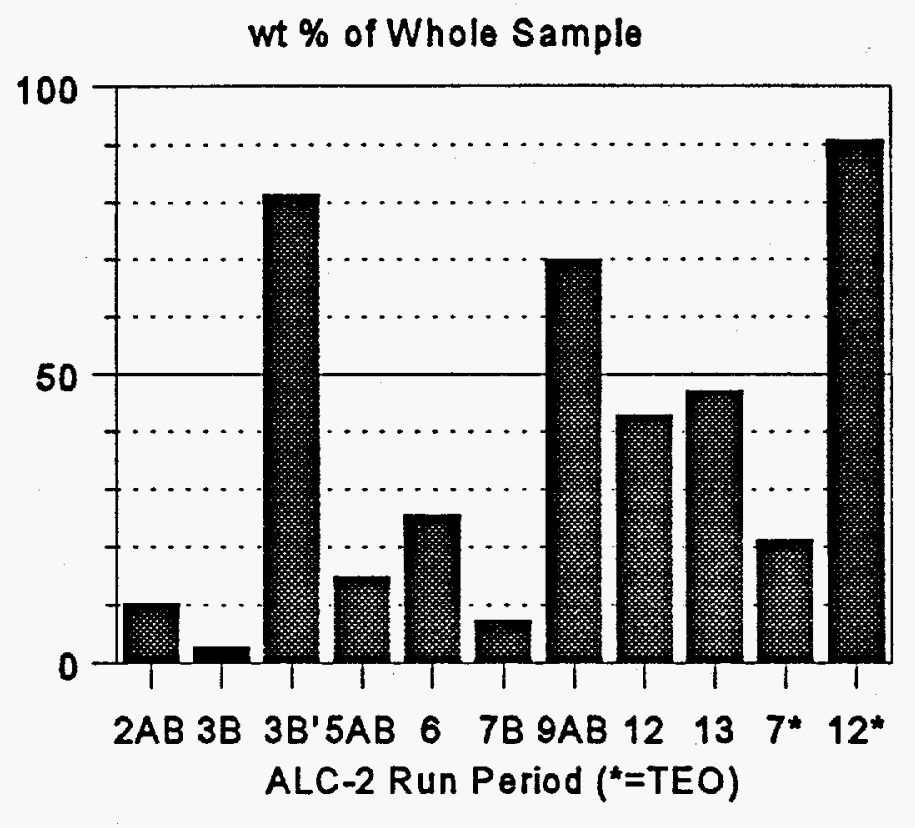

Toluene

Figure 1. Toluene Concentration of As-Received TES and TEO Samples.

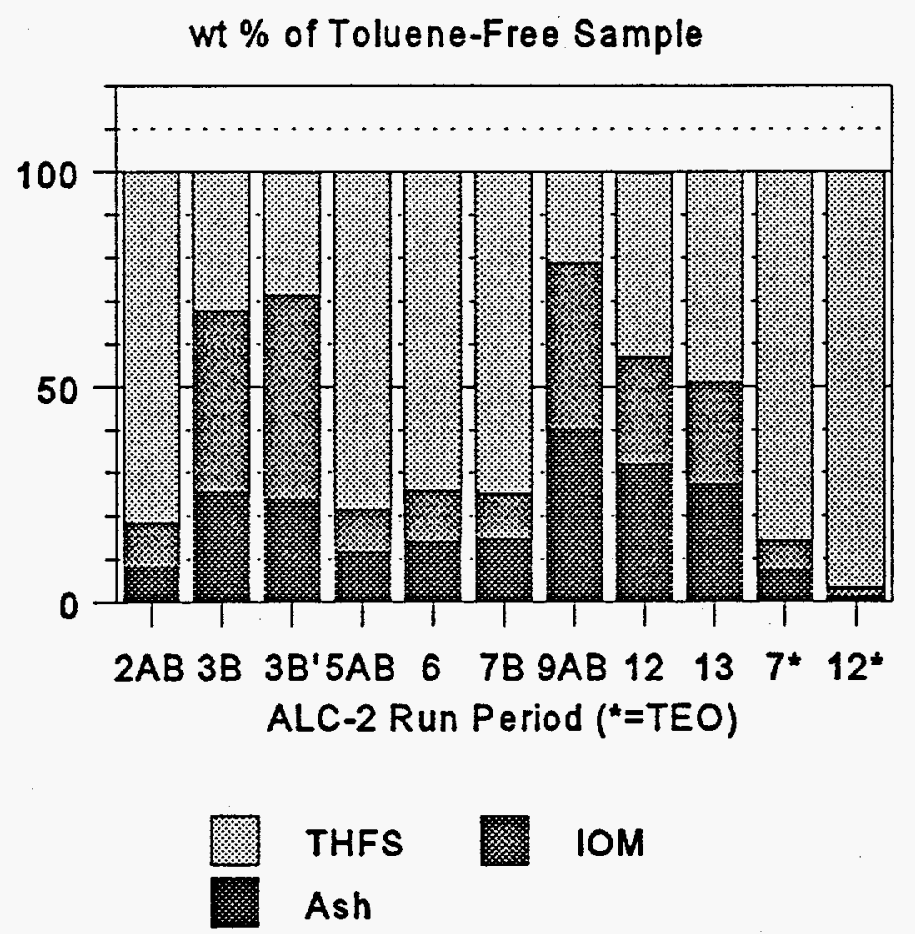

Figure 2. Composition of Toluene-Free TES and TEO Samples. 


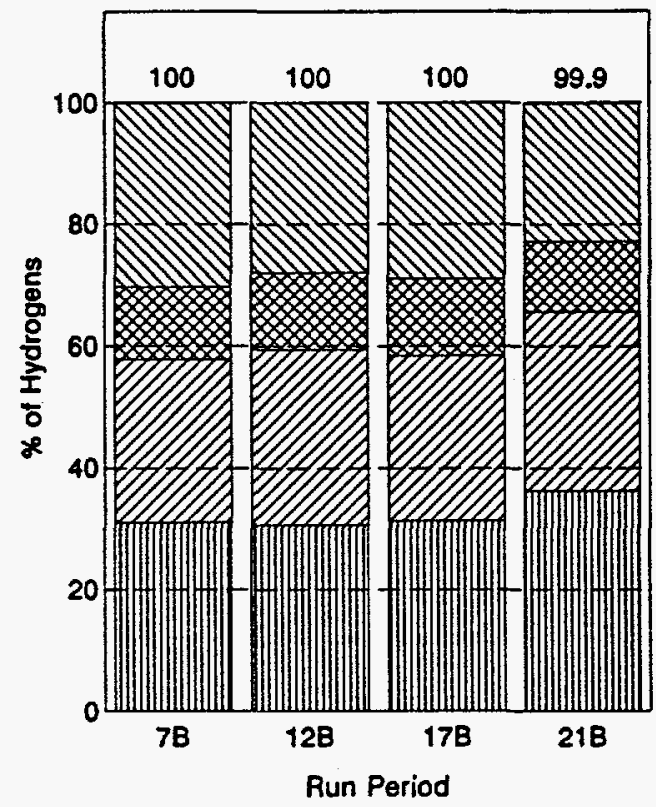

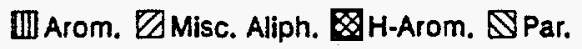

Figure 3. HTI Run ALC-2, Proton Distributions of Filtered Whole O-6 Bottoms Samples

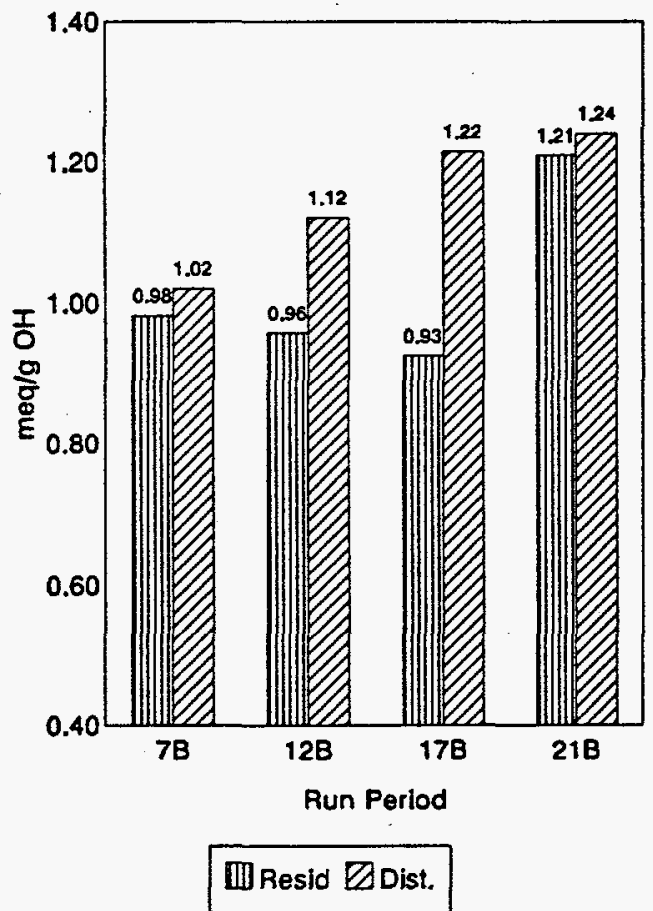

Figure 4. HTI Run ALC-2, Phenolic -OH Concentrations in 0-6 Bottoms $454^{\circ} \mathrm{C}-$ Distillate and $454^{\circ} \mathrm{C}^{+}$Resid Samples. 


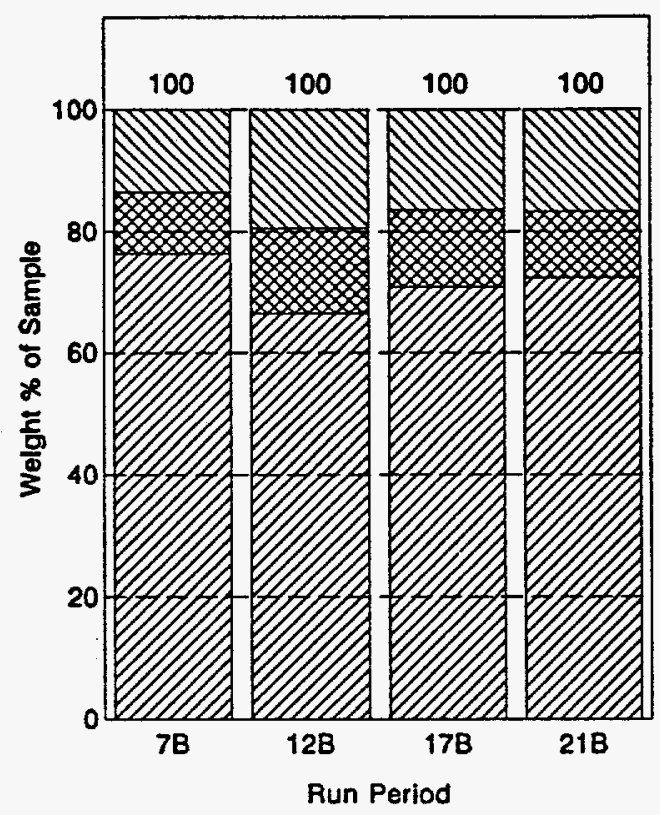

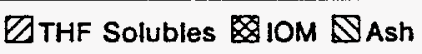

Figure 5. HTI Run ALC-2, Composition of Whole CVSB Samples.

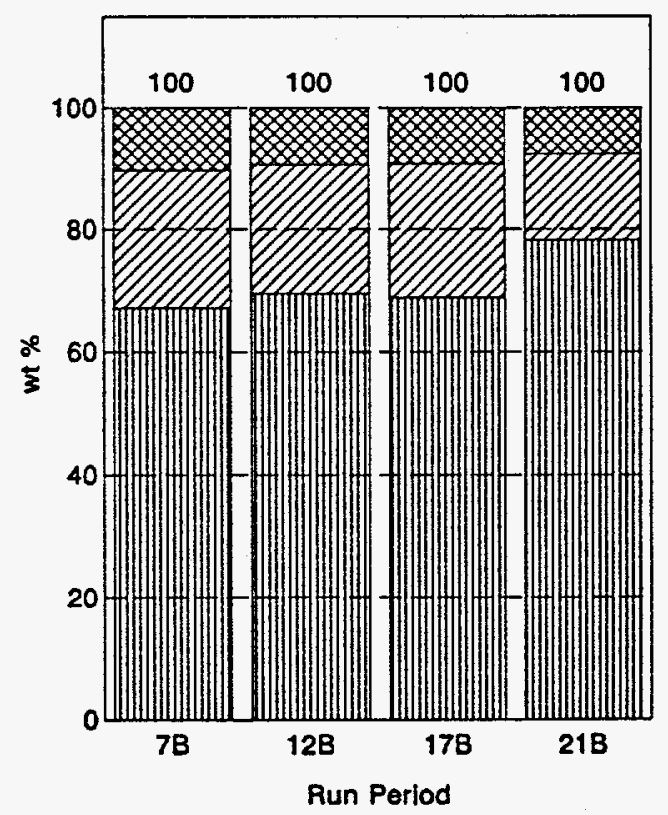

而Oils $\square$ Asphaltenes. Preasphaltenes

Figure 6. HTI Run ALC-2, Solubility Fractions of THF-Soluble Portion of CVSB Samples. 


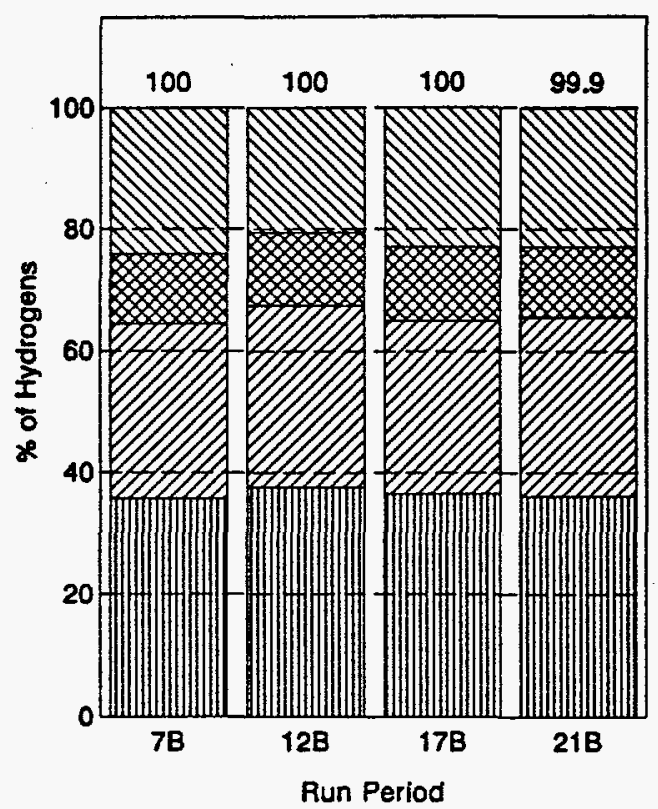

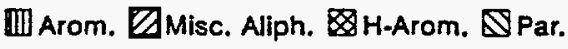

Figure 7. HTI Run ALC-2, Proton Distributions of Filtered Whole CVSB Samples.

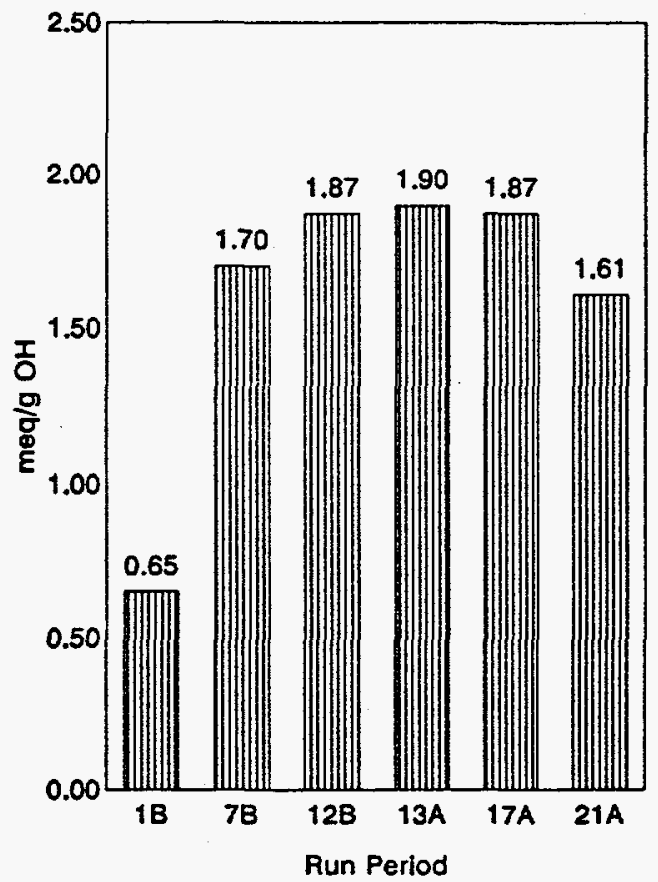

Figure 8. HTI Run ALC-2, Phenolic -OH Concentrations in First-Stage SOH Oils. 


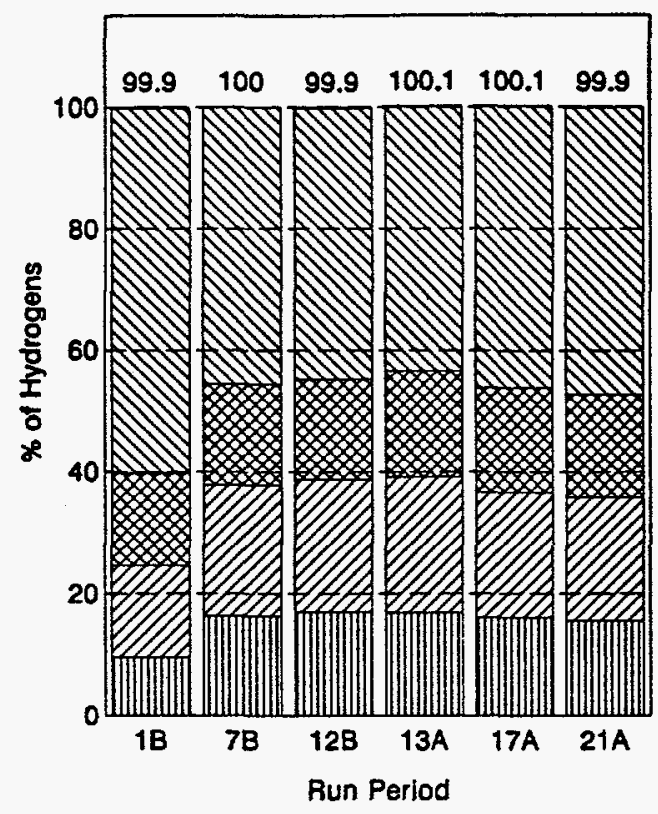

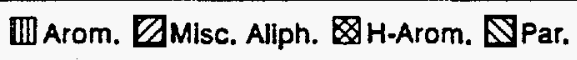

Figure 9. HTI Run ALC-2, Proton Distributions of First-Stage SOH Oils.

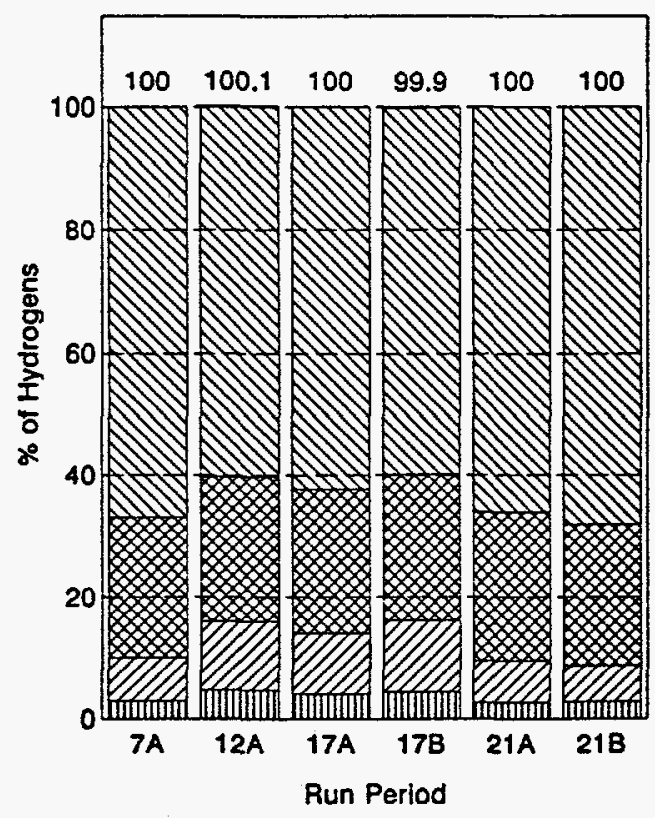

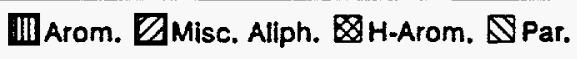

Figure 10. HTI Run ALC-2, Proton Distributions of Second-Stage SOH Oils. 


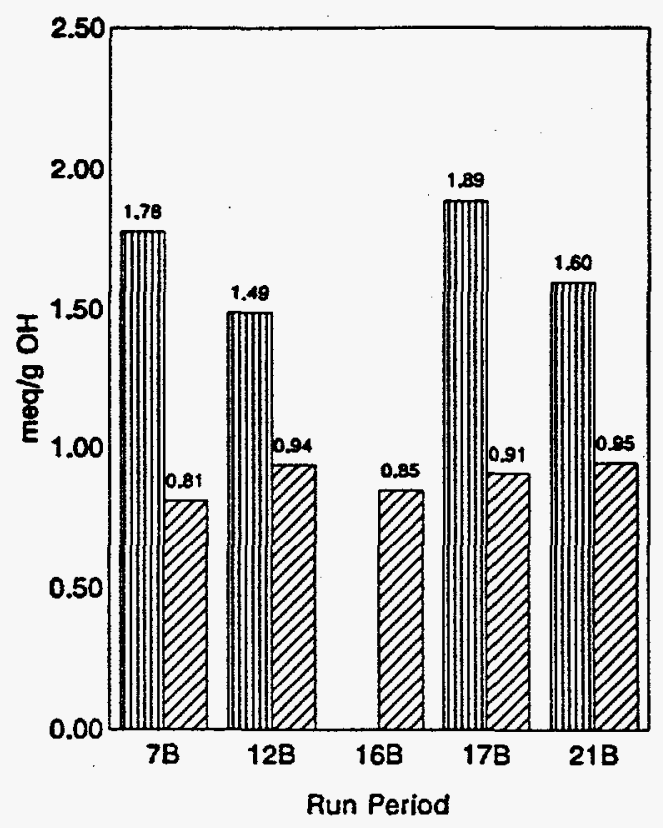

III RPSOH $\square$ RPSB

Figure 11. HTI Run ALC-2, Phenolic -OH Concentrations in RPSOH and RPSB Oils.

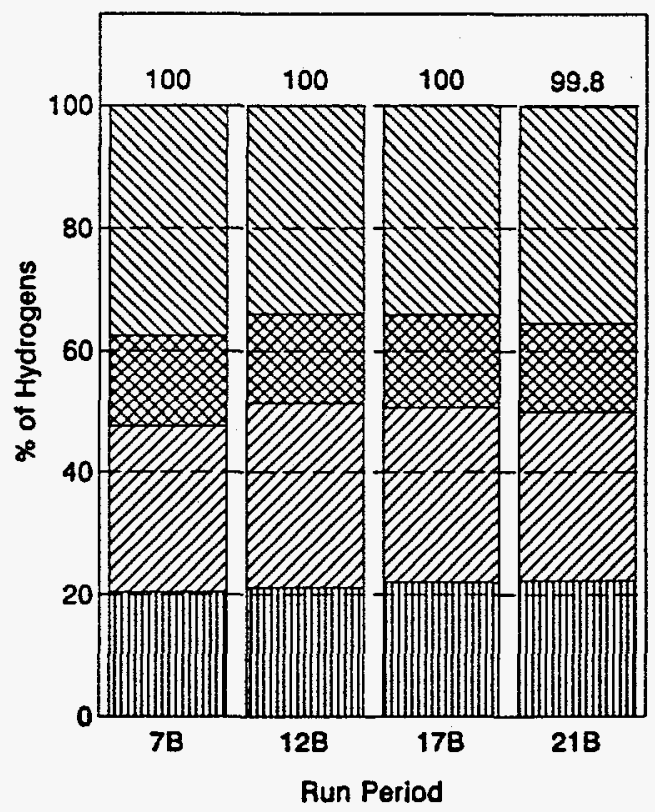

而Arom. $\triangle$ Misc. Aliph. $\otimes$ H-Arom. $\$$ Par.

Figure 12. HTI Run ALC-2, Proton Distributions of RPSOH Oils. 


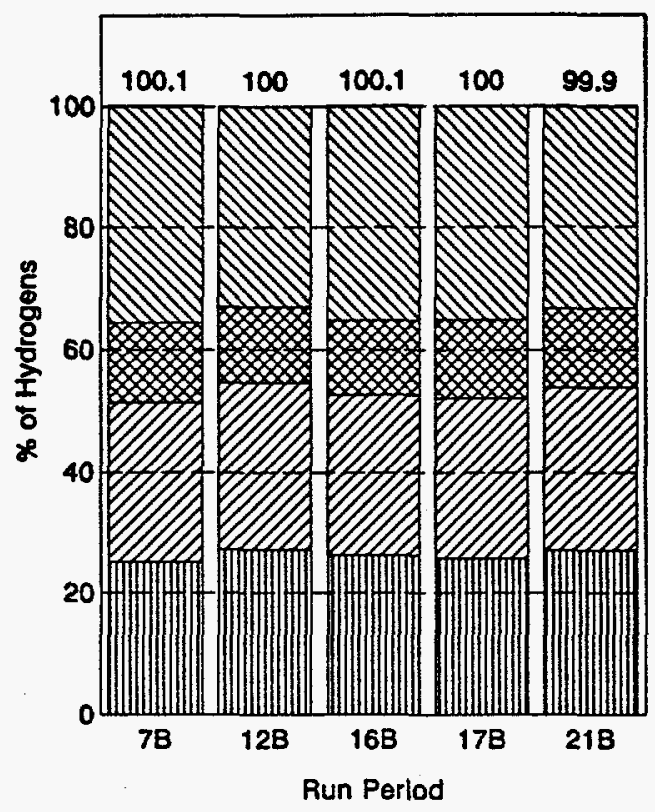

III Arom. $\triangle$ Misc. Allph. H-Arom. $\mathrm{QPar}$

Figure 13. HTI Run ALC-2, Proton Distributions of RPSB Samples. 


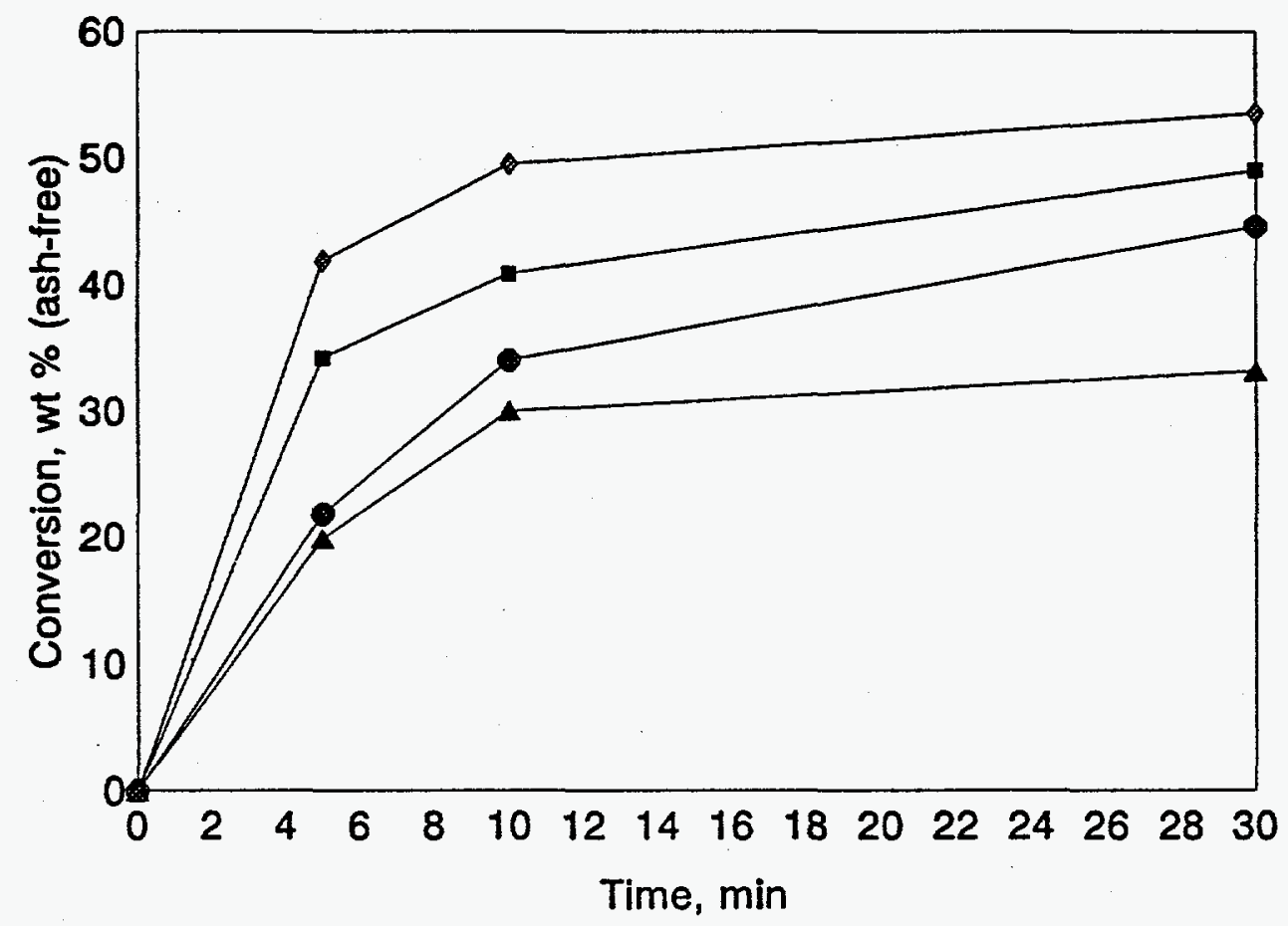

$\triangle$ Resid C $420^{\circ} \mathrm{C} \rightarrow$ Resid C $435^{\circ} \mathrm{C}-$ Resid $\mathrm{H} 420^{\circ} \mathrm{C} \diamond$ Resid H $435^{\circ} \mathrm{C}$

Figure 14. Conversion vs Time for the Catalyzed Hydroprocessing of Resid $C$ and Resid $H$ (1500 psig $\mathrm{H}_{2} ; 3$ wt \% Mo; Tetralin:Resid = 3:1 wt ratio) 


\section{APPENDIX 1}

CALEB BRETT REPORT ON CRUDE OIL ASSAY OF NET PRODUCTS OF HTI RUN PB-05 


\section{TTS Intertek Testing Services}

Caleb Brett

\author{
9809 Rowlett Road \\ Houston, TX 77075 \\ Phone: (713) 844-3200 \\ Fax: (713) 844-3330
}

\section{Laboratory Report No. 97-000431-0-HOUS; 1}

Consol, Inc.

Research \& Development

4000 Brownsville Road

Library, PA $15129-9566$

For the Attention of R.A. Winschel

SAMPLE DETAILS: 8 cut(s) from one sample received on 10-JAN-1997

SOURCE : Consol, Inc.

CUSTOMER PRODUCT

DESCRIPTION

LAB REE

"HTI PB-05-22,23,24,25 CRUDE OIL"

Sample As Received

$001-00$

IBP-70 Deg. F

70-180 Deg. F

180-350 Deg. F

350-400 Deg. F

400-550 Deg. F

550-650 Deg. F

002-00

$003-00$

004-00

005-00

006-00

$650+$ Deg. F

007-00

008-00

CONTAINERS

RESULTS
5 Galion Can

SEE ATTACHED SHEETS
SEAIS : NONE

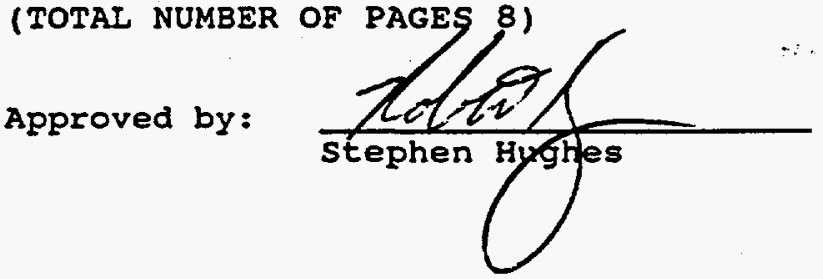




\section{TTS Intertek Testing Services Caleb Brett}

Laboratory Report No. 97-000431-0-Hous; 1 - Page 2 of 8

Sample ID

97-000431-0-HOUS-001-00

Test

API Gravity e 60/60 F

Specific Gravity e $60 / 60 \mathrm{~F}$

Carbon

Hydrogen

Nitrogen

Oxygen (By Difference)

Sulfur Content

Total Nitrogen

Ash Content for Digestion

Vanadium

Iron

Nickel

Freezing Point

Microcarbon Residue

N-Heptane Insolubles

Boiling Range Distribution

Initial Boiling Point

e 58 Recovery

e 108 Recovery

e 208 Recovery

e $30 \%$ Recovery

e 408 Recovery

e $50 \%$ Recovery

e 608 Recovery

e 708 Recovery

e $80 \%$ Recovery

e 908 Recovery

e 958 Recovery

Final Boiling point

Recovery

Residue

Loss

\section{Customer Product Description}

"HTI PB-05-22,23,24,25 CRUDE OIL"

Sample As Received

Method

001-00

2-API

33.9

Wt. 8

Wt. 8

Wt. 8

Wt. \&

Wt. \&

ppm

WE. \&

Ppm

ppm

ppm

Deg. F

Wt. \&

Wt. \&

D5291

0.8553

85.44

11.83

0.48

1.77

D4294

D4629

0.48

D482

ICP

D2386

D4530

D3279

D2887

Deg. F

Deg. F

Deg. F

Deg. F

Deg. F

Deg. F

Deg. F

Deg. F

Deg. F

Deg. F

Deg. F

Deg. F

Deg. F

vol. :

Vol. \&

vol. :
4791.0

$<0.001$

1.2

1.2

0.4

Too Dark

0.40

0.01

See Attached

123

197

237

284

325

368

412

455

495

544

633

673

685

96.8

1.2

2.0
Sample ID

97-000431-0-HOUS-002-00

Test

API Gravity a 60/60 F Specific Gravity e $60 / 60 \mathrm{~F}$ DHA
Customer Product Description

"HTI PB-05-22,23,24,25 CRUDE OIL"

IBP-70 Deg. F
Method

002-00

2-API

111.4

0.5826

GC-DHA

See Attached 


\section{TTS Intertek Testing Services}

Caleb Brett

Laboratory Report No. 97-000431-0-Hous; 1 - Page 3 of 8

Sample ID

97-000431-0-HOUS-003-00

Test

API Gravity $60 / 60 \mathrm{~F}$ Specific Gravity e $60 / 60 \mathrm{~F}$ Carbon

Hydrogen

Nitrogen

Oxygen (BY Difference)

Sulfur Content

Total Nitrogen

Vapor Pressure

Paraffins

Olefins

Naphthenes

Aromatics

Total $N \& A$

Total Acid Number

Corrosion 3 hrs a 122 F

Existent Gum

Oxidation Stability

Research Octane Number

Motor Octane Number

Initial Boiling Point

e 5 Evaporated

e $10 z$ Evaporated

e $20 \%$ Evaporated

e 308 Evaporated

e $40 \%$ Evaporated

e $50 \%$ Evaporated

e 60\% Evaporated

e 708 Evaporated

e 808 Evaporated

e 908 Evaporated

e 958 Evaporated

Final Boiling Point

Recovery

Residue

Loss

Benzene
Customer Product Description

"HTI PB-05-22,23,24,25 CRUDE OIL"

70-180 Deg. F
$003-00$

75.0

0.6854

84.28

15.51

0.12

$<.01$

$D 4294$

D4629

D 323

GC-PONA

0.09

1190.0

9.6

poi. o

Vol. :

Vol. \&

Vol. \&

Vol. \&

$\mathrm{mgKOH} / \mathrm{g}$

D 974

D130

$\mathrm{mg} / 100 \mathrm{~mL} \quad$ D381

min.

D525

D2699

D2700

Deg. F

D86

63.87

8.74

24.11

3.33

27.44

0.041

$4 a$

$>240$

70.8

66.0

100

120

124

129

134

138

144

149

155

161

170

178

194

97.1

0.8

2.1

Vol. \&

Vol. \&

Wt. \&

GC

2.64 


\section{Intertek Testing Services}

Caleb Brett

Laboratory Report No. 97-000431-0-HOUS; 1 - Page 4 of 8

Sample ID

97-000431-0-HOUS-004-00

Test

API Gravity e $60 / 60 \mathrm{~F}$ Specific Gravity e $60 / 60 \mathrm{~F}$ Carbon

Hydrogen

Nitrogen

Oxygen (By Difference)

Sulfur Content

Total Nitrogen

Mercaptan sulfur content

Vapor Pressure

Paraffins

Olefins

Naphthenes

Aromatics.

Total N\& A

Total Acid Number

Corrosion 3 hrs e 122 F

Existent Gum

Oxidation Stability

Research Octane Number

Motor Octane Number

Initial Boiling Point

e $5 \%$ Evaporated

e 108 Evaporated

e 208 Evaporated

e $30 \%$ Evaporated

a $40 \%$ Evaporated

e 508 Evaporated

e 608 Evaporated

e 708 Evaporated

e 808 Evaporated

e 908 Evaporated

C 958 Evaporated

Final Boiling Point

Recovery

Residue

Loss
Customer Product Description

"HTI PB-05-22, 23,24,25 CRUDE OIL"

180-350 Deg. F
Wt. \&

Wt. \&

Wt. \&

WE. \&

WE. \&

ppm

ppm

psi

Vol. \&

Vol. \&

Vol. \&

Vol. \&

$\mathrm{mgKOH} / \mathrm{g}$

$\mathrm{mg} / 100 \mathrm{~mL}$

min.

Deg. F

Deg. F

Deg. F

Deg. F

Deg. F

Deg. F

Deg. F

Deg. F

Deg. F

Deg. $F$

Deg. F

Deg. F

Deg. F

Vol. \&

Vol. \&

vol. \& vol. \&
Method

2-API

D5291

004-00

48.5

0.7862

85.47

13.18

0.15

1.02

D4294

D4629

UOP 163

D323

0.18

1543.0

516

1.5

41.21

4.01

30.31

24.27

54.58

D974

D130

D381

D525

D2699

D2700

D86

0.011

$3 a$

16
$>240$

66.0

60.8

196
235

242

248

255

263

272

281

291

302

315

325

337

98.7

0.8

0.5 


\section{TTS Intertek Testing Services Caleb Brett}

Laboratory Report No. 97-000431-0-HoUs; 1 - Page 5 of 8

Sample ID

97-000431-0-HOUS-005-00

Tegt

API Gravity @ $60 / 60 \mathrm{~F}$ Specific Gravity @ $60 / 60 \mathrm{~F}$ Carbon

Hydrogen

Nitrogen

oxygen (By Difference)

Sulfur Content

Total Nitrogen

Mercaptan sulfur content

Viscosity a $-20 \mathrm{C}$

Viscosity e $100 \mathrm{C}$

Freezing Point

Pour Point

Flash Point, TCC

Vapor Pressure

Luminometer Number

Smoke Point

Paraffins

Olefins

Naphthenes

Aromatics

Total N\& $A$

Naphthalenes

Total Acid Number

Corrosion 3 hrs e 122

Existent Gum

Pressure Drop

Oxidation stability

Research Octane Number

Motor Octane Number

Cetane Number

Initial Boiling point

e 58 Evaporated

e 10\% Evaporated

e 20: Evaporated

(e 30\% Evaporated

(e) 408 Evaporated

e 508 Evaporated

e 60\% Evaporated

e 708 Evaporated

e 80\% Evaporated

e 908 Evaporated

e 958 Evaporated

Final Boiling point

Recovery

Residue

Loss

Net Heat of Combustion
Customer Product Description

"HTI PB-05-22,23,24,25 CRUDE OIL"

350-400 Deg. F

Method

$\underline{005-00}$

2-API

33.7

Wt. o

D5291

0.8565

Wt. \&

Wt. \&

Wt. \&

Wt. \&

D4294

ppm

D4629

UOP 163

ppm

cst

D445

cSt

Deg. F

Deg. F

Deg. F

D2386

D97

D56

psí

D323

D 1740

$\mathrm{mm}$

D1322

Vol. \%

Vol. \&

GC-PONA

84.64

11.83

0.57

2.79

0.18

5658.0

214

6.460

0.664

$-68.8$

$<-85.0$

136

0.1

36.0

15

32.47

6.80

Vol. \&

Vol. *

Vol. \&

Vol. \%

$\mathrm{mgKOH} / \mathrm{g}$

D1840

D974

D130

$\mathrm{mg} / 100 \mathrm{~mL} \quad \mathrm{D} 381$

$\mathrm{mmHg}$

D 3241

min.

D525

D2699

D2700

D613

Deg. F

Deg. F

Deg. F

D86

18.66

42.07

60.73

4.39

0.04

$1 \mathrm{a}$

21

$>125.0$

$>240$

92.0

79.2

19.4

250

355

355

357

358

Deg. F

Deg. F

Deg. F

Deg. F

Deg. F

Deg. F

Deg. F

Deg. F

Deg. F

Vol. 8

Vol. \&

Vol. \&

BTU/1b
360

362

364

367

371

377

382

392

98.7

1.3

0.0

18551 
TTS Intertek Testing Services

Caleb Brett

Laboratory Report No. 97-000431-0-HoUs; 1 - Page 6 of 8

Sample ID

97-000431-0-HOUS-006-00

Tegt

API Gravity e 60/60 F Specific Gravity e $60 / 60 \mathrm{~F}$ Carbon

Hydrogen

Nitrogen

oxygen (By Difference)

Sulfur Content

Total Nitrogen

Basic Nitrogen

Mercaptan Sulfur Content

Viscosity e $-20 \mathrm{c}$

viscosity e $40 \mathrm{C}$

Viscosity @ $100 \mathrm{C}$

Freezing Point

Pour Point

Aniline Point

Flash Point, TCC

Luminometer Number

Smoke Point

Paraffins

Olefins

Naphthenes

Aromatics

Total N\&A

Naphthalenes

Total Acid Number

Corrosion 3 hrs e 122 F

Existent Gum

Pressure Drop

Cetane Number

Initial Boiling point

e 58 Evaporated

e 108 Evaporated

e 208 Evaporated

C 308 Evaporated

C 408 Evaporated

c 508 Evaporated

e 608 Evaporated

e 708 Evaporated

e 808 Evaporated

C 908 Evaporated

e $95 \%$ Evaporated

Final Boiling Point

Recovery

Residue

Loss

Net Heat of Combustion
Customer Product Description

"HTI PB-05-22, 23,24,25 CRUDE OIL"

400-550 Deg. F

Method

$\underline{006-00}$

2-API

26.9

0.8932

Wt. \&

D5291

Wt. 8

WE. \&

Wt. \&

Wt. \&

ppm

ppm

ppm

cst

cSt

cSt

Deg. F

Deg. F

Deg. $E$

Deg. F

$\mathrm{mm}$

Vol. \&

vol. \&

vol. \&

vol. \&

vol. \&

vol. 8

mgKOH/g

D1840

D4294

D4629

UOP 269

UOP 163

D445

D2386

D97

D611

D56

D1740

D1322

GC-PONA

$\mathrm{mg} / 100 \mathrm{~mL}$

D974

D130

D381

$\mathrm{mmHg}$

D3241

D613

Deg. F

Deg. F

Deg. F

Deg. F

Deg. F

Deg. F

Deg. F

Deg. F

Deg. F

Deg. F

Deg. F

Deg. F

Deg. F

Vol. \&

Vol. \&

Vol. \&

BTU/Lb
85.48

11.26

0.74

2.05

0.47

7373.0

5319

262

19.83

2.509

0.969

$-24.7$

$-27.0$

92.5

200

22.0

10

22.73

8.60

16.87

51.80

68.67

7.02

0.042

$1 \mathbf{a}$

$>125.0$

19.6

425

436

438

442

446

451

458

465

475

486

500

514

525

98.4

0.8

0.8

18294 


\section{TTS Intertek Testing Services Caleb Brett}

Laboratory Report No. 97-000431-0-HoUs; 1 - Page 7 of 8

Sample ID

97-000431-0-HOUS-007-00

Test

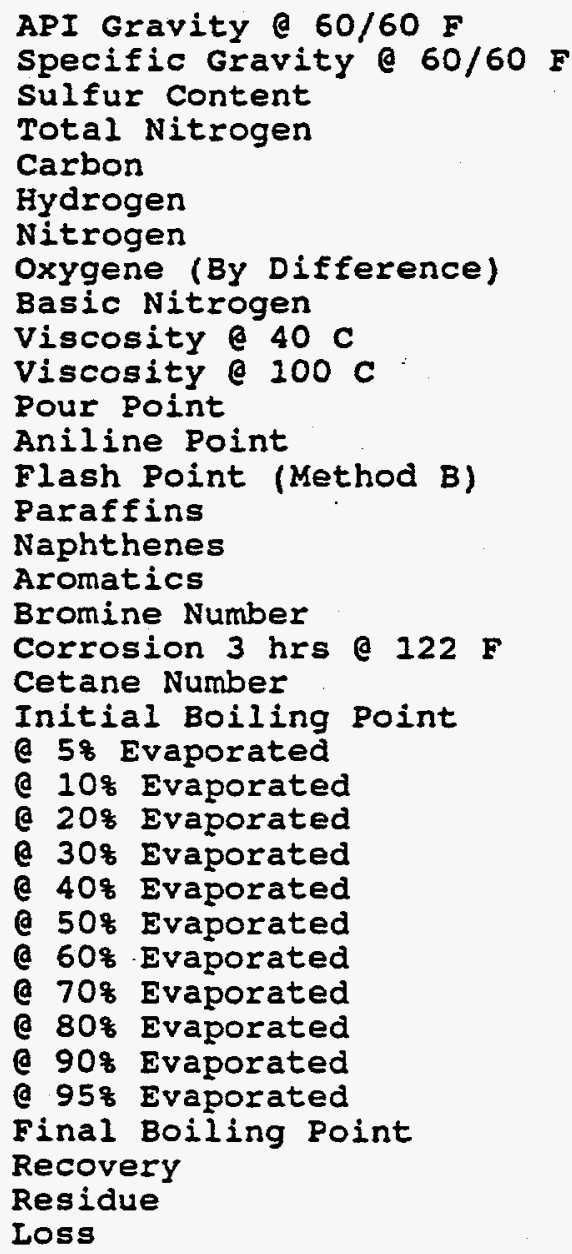

Customer Product Description

"HTI PB-05-22,23,24,25 CRUDE OIL"

550-650 Deg. F
Method

007-00

2-API

19.8

wt. 8

ppm

Wt. \&

Wt. \&

Wt. \&

Wt. \&

ppm

cst

cst

Deg. F

Deg. F

Deg. F

Vol. 8

Vol. \&

Vol. \&

D4294

D4629

D5291

UOP2 69

D445

D97

D611

D93

GC-PNA

D1159

D130

D613

Deg. $F$

Deg. F

Deg. F

Deg. F

Deg. F

Deg. F

Deg. F

Deg. F

Deg. F

Deg. F

Deg. F

Deg. $F$

Deg. F

Vol. \&

Vol. \&

D86
0.9355

0.59

6114.0

86.62

10.58

0.61

1.6

3503

10.04

1.837

32.0

108.0

$>200$

25.33

14.55

60.12

16.0

$1 a$

26.9

557

573

576

577

579

580

582

585

589

594

603

610

621

97.7

1.1

1.2 


\section{TTS Intertek Testing Services Caleb Brett}

Laboratory Report No. 97-000431-0-HOUS; 1 - Page 8 of 8

Sample ID

97-000431-0-HOUS-008-00

Test

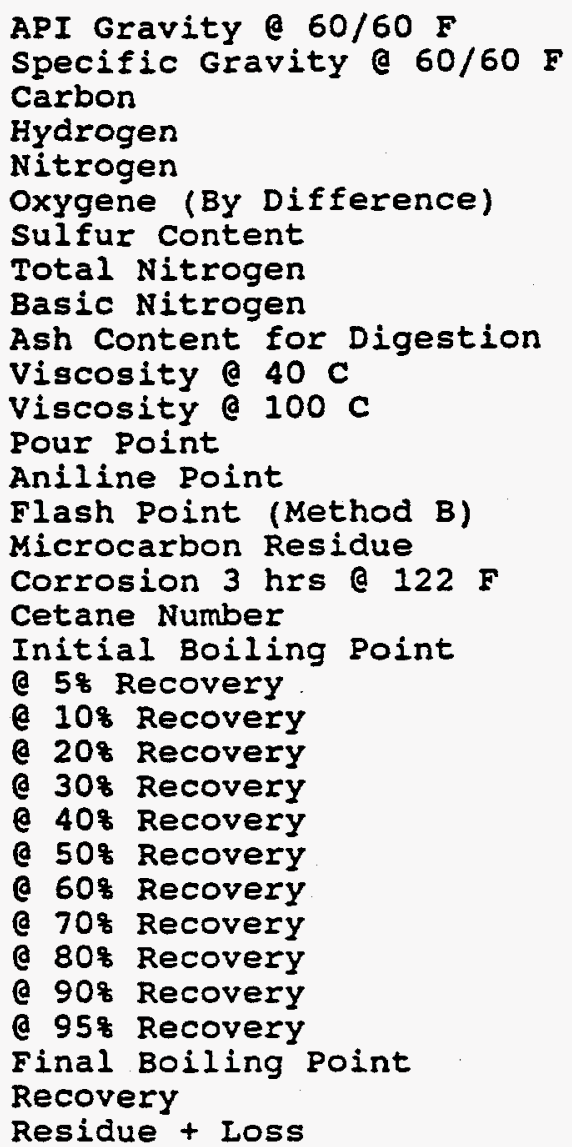

\section{Customer Product Description}

"HTI PB-05-22,23,24,25 CRUDE OIL"

$650+$ Deg. F

* Note * The boiling point of this fraction is out of the scope of the method. (Fraction is too heavy).

Method

2-API

D5291

Wt. \&

Wt. \&

Wt. \&

Wt. 8

Wt. \&

ppm

ppm

Wt. \&

cst

cst

Deg. F

Deg. F

Deg. F

Wt. \&

Deg. F

Deg. F

Deg. F

Deg. F

Deg. F

Deg. F

Deg. F

Deg. F

Deg. F

Deg. F

Deg. F

Deg. F

Deg. $F$

Vol. :

Vol. \&

D4294

D4629

UOP 269

D482

D445

D97

D611

D93

D4530

D130

D613

D1160
008-00

11.1

0.9923

88.55

9.67

0.75

$<.01$

1.03

7543.0

3630

0.119

34.36

4.472

75.0

131.0

$>200$

1.80

* See Note *

660

673

673

681

684

689

696

706

719

738

797

873

980

97.0

3. 0 
TID: 97-000431-0-HOUS-001-00

CID: CONSOLINC

SID: HTI PBO5-22,23,24,25 CRUDE OIL/AS RECEIVED

NID: 58102

Boiling Point Distribution ASTM D-2887

\begin{tabular}{|c|c|c|c|c|c|c|c|c|}
\hline$\%$ Off & $\mathrm{BP}(\mathrm{F})$ & $\mathrm{BP}(\mathrm{C})$ & $\%$ Off & $\mathrm{BP}(\mathrm{F})$ & $B P(C)$ & $\%$ off & $\mathrm{BP}(\mathrm{F})$ & $B P(C)$ \\
\hline$\overline{\text { IBP }}$ & 84.2 & 29.0 & 40 & $\overline{384.2}$ & 195.7 & 80 & 568.5 & 298.1 \\
\hline 1 & 91.3 & 32.9 & 41 & 388.4 & 198.0 & 81 & 573.2 & 300.7 \\
\hline 2 & 146.0 & 63.3 & 42 & 390.5 & 199.2 & 82 & 577.0 & 302.8 \\
\hline .3 & 158.0 & 70.0 & 43 & 395.3 & 201.8 & 83 & 584.0 & 306.7 \\
\hline 4 & 167.8 & 75.4 & 44 & 399.2 & 204.0 & 84 & 589.9 & 309.9 \\
\hline 5 & 181.6 & 83.1 & 45 & 402.6 & 205.9 & 85 & 596.8 & 313.8 \\
\hline 6 & 191.3 & 88.5 & 46 & 409.0 & 209.4 & 86 & 600.8 & 316.0 \\
\hline 7 & 205.5 & 96.4 & 47 & 415.2 & 212.9 & 87 & 607.9 & 319.9 \\
\hline 8 & 209.8 & 98.8 & 48 & 416.9 & 213.8 & 88 & 615.4 & 324.1 \\
\hline 9 & 214.7 & 101.5 & 49 & 422.0 & 216.7 & 89 & 622.2 & 327.9 \\
\hline 10 & 224.5 & 106.9 & 50 & 426.6 & 219.2 & 90 & 630.0 & 332.2 \\
\hline 11 & 228.5 & 109.2 & 51 & 430.9 & 221.6 & 91 & 638.3 & 336.8 \\
\hline 12 & 235.0 & 112.8 & 52 & 434.4 & 223.6 & 92 & 646.2 & 341.2 \\
\hline 13 & 239.5 & 115.3 & 53 & 437.2 & 225.1 & 93 & 654.7 & 345.9 \\
\hline 14 & 253.0 & 122.8 & 54 & 443.2 & 228.4 & 94 & 667.5 & 353.1 \\
\hline 15 & 255.7 & 124.3 & 55 & 448.1 & 231.2 & 95 & 677.5 & 358.6 \\
\hline 16 & 265.3 & 129.6 & 56 & 452.4 & 233.6 & 96 & 691.9 & 366.6 \\
\hline 17 & 268.3 & 131.3 & 57 & 455.2 & 235.1 & 97 & 712.4 & 378.0 \\
\hline 18 & 272.8 & 133.8 & 58 & 459.4 & 237.4 & 98 & 738.8 & 392.7 \\
\hline 19 & 273.6 & 134.2 & 59 & 463.2 & 239.6 & 99 & 810.1 & 432.3 \\
\hline 20 & 277.0 & 136.1 & 60 & 467.8 & 242.1 & FBP & 1238.2 & 670.1 \\
\hline
\end{tabular}

Start Time: 0.2 minutes End Time: 44.7 minutes Area: 124898168.0

Slice Width: $0.80 \mathrm{sec}$
140.2

142.5

147.7 .

149.1

154.0

156.9

159.7

163.7

165.1

168.9

171.4

173.5

177.3

179.7

183.4

184.8

185.7

190.2

193.0
471.3

475.3

479.4

481.7

485.2

490.8

497.2

501.9

509.0

513.7

518.5

524.1

529.7

534.6

541.4

545.4

551.2

557.6

244.1

246.3

248.6

249.8

251.8

254.9

258.4

261.1

265.0

267.6

270.3

273.4

276.5

279.2

283.0

285.2

288.4

292.0

294.7
Sample Offset: 8908.0

Baseline Offset: 8422.0

Calibration File: 0312raa

Calibration Date: 03/13/97

Baseline Subtracted: C:ITC4ISD689010312BA 
- CALEB BREIT HOUSTUN

TID: 97-000431-0-HOUS-001-00

CID: CONSOLINC

SID: HTI PBO5-22,23,24,25 CRUDE OIL/AS RECEIVED

NID: 58102 Date: 10-JAN-1997

Calibration Plot

ASTM D-2887

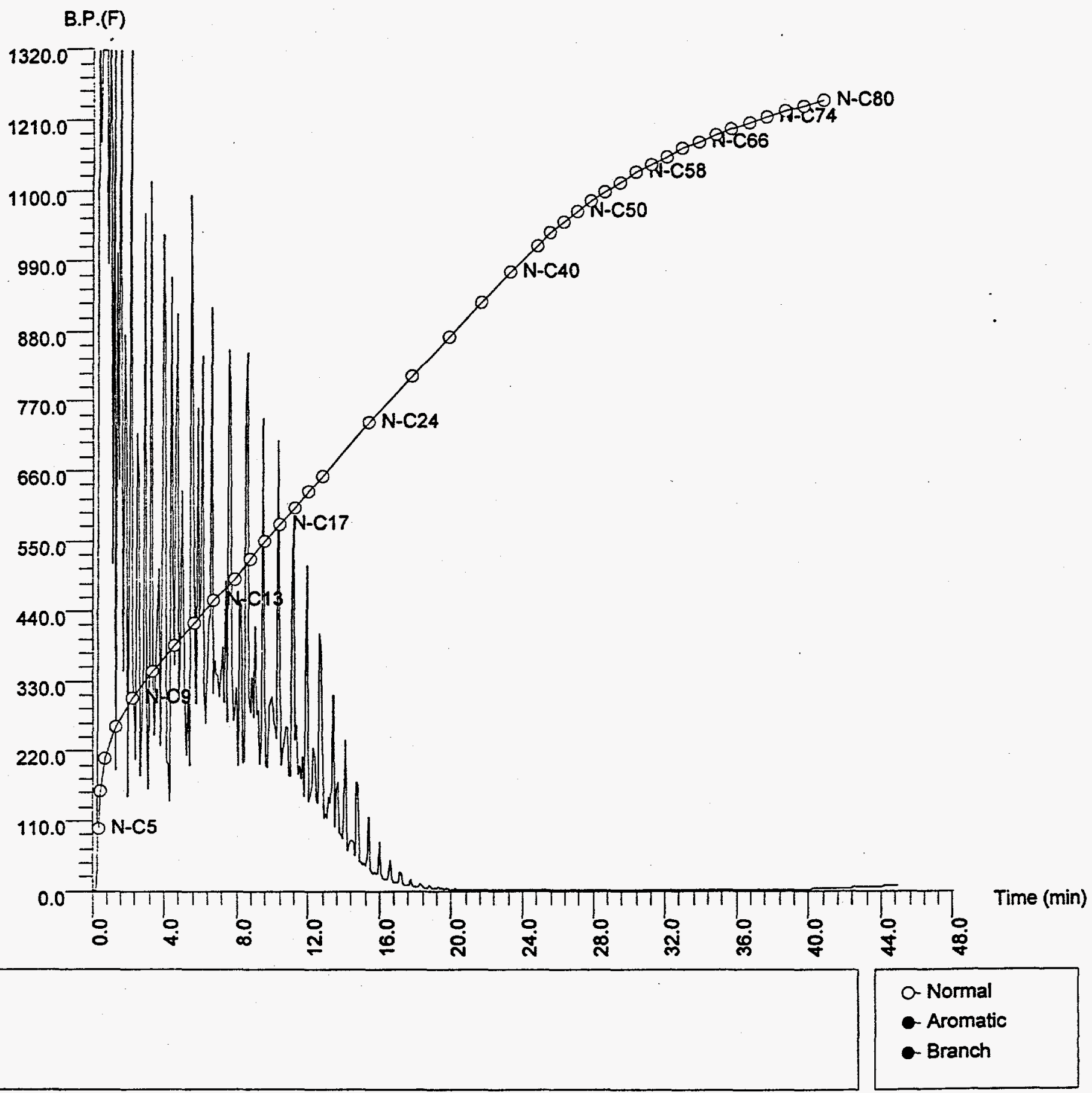




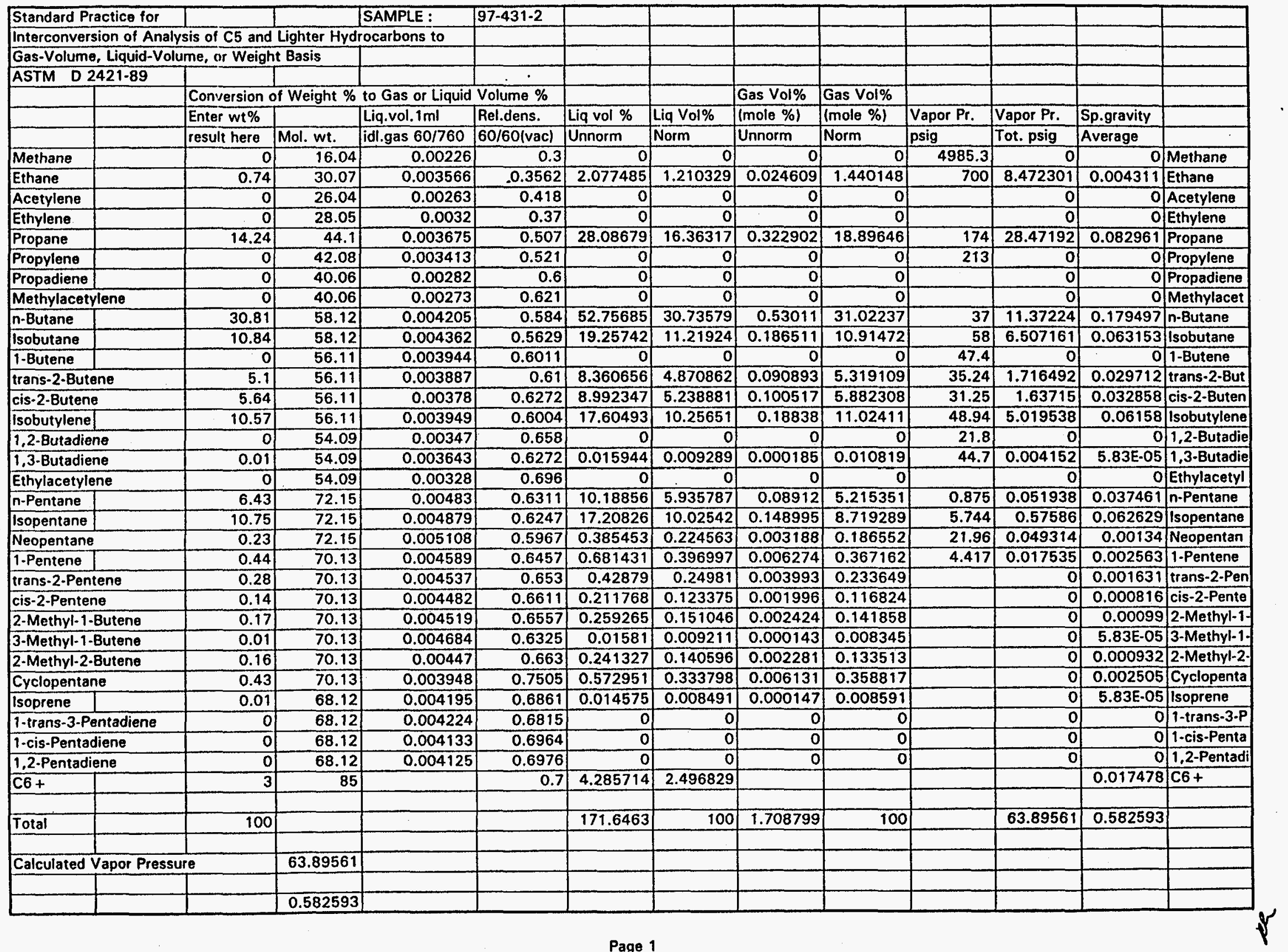


- CALEB BRETT HUUSTUN

TID: 97-000431-0-HOUS-002-00

CID: CONSOLINC

SID: HTI PBO5-22,23,24,25 CRUDE OIL/IBP-70 F

NID: 58159

Date: 10-JAN-1997

Analyzed: 1/29/97 9:49 AM

Reported: 01-29-1997 11:45:10 Normalized to $100.00 \%$

Comments :

Components Iisted in Chromatographic Order

$\begin{array}{lll}\text { Min. } & \text { INDEX } & \text { Component } \\ 6.592 & 182.7 & \text { ethylene } \\ 6.656 & 200.0 & \text { ethane } \\ 7.086 & 300.0 & \text { propane } \\ 7.677 & 347.6 & ? \\ 7.777 & 353.3 & ? \\ 8.088 & 368.5 & \text { i-butane } \\ 8.729 & 392.3 & \text { isobutylene } \\ 8.988 & 400.0 & \text { n-butane } \\ 9.438 & 414.7 & \text { t-butene-2 } \\ 9.525 & 417.3 & 2,2 \text {-dimethylpropane } \\ 9.779 & 424.5 & \text { ? } \\ 9.946 & 428.9 & \text { c-butene-2 } \\ 10.302 & 437.7 & ? \\ 11.129 & 455.2 & \text { ethanol } \\ 11.411 & 460.5 & 3 \text {-methylbutene-1 } \\ 12.479 & 478.1 & \text { i-pentane } \\ 13.321 & 489.9 & \text { pentene-1 } \\ 13.481 & 491.9 & \text { i-propanol } \\ 13.582 & 493.2 & ? \\ 13.775 & 495.6 & 2 \text {-methylbutene-1 } \\ 14.138 & 500.0 & \text { n-pentane } \\ 14.353 & 504.1 & \text { isoprene } \\ 14.607 & 508.9 & \text { t-pentene-2 } \\ 14.948 & 515.0 & 3,3-\text { dimethylbutene-1 } \\ 15.112 & 517.9 & \text { c-pentene-2 } \\ 15.437 & 523.4 & 2 \text {-methylbutene-2 } \\ 16.437 & 539.2 & 2,2-\text { dimethylbutane } \\ 17.504 & 554.4 & \text { O6 } \\ 17.647 & 556.3 & \text { cyclopentene } \\ 18.017 & 561.2 & 4 \text {-methylpentene-1 } \\ 18.084 & 562.0 & 3 \text {-methylpentene-1 } \\ 18.419 & 566.3 & \text { cyclopentane } \\ 18.613 & 568.7 & 2,3-\text { dimethylbutane } \\ 18.751 & 570.4 & 4-\text { methyl-c-pentene-2 } \\ 18.895 & 572.1 & ? \\ 19.001 & 573.4 & 2 \text {-methylpentane } \\ 19.120 & 574.8 & 4 \text {-methyl-t-pentene-2 } \\ 20.020 & 585.1 & 3 \text {-methylpentane } \\ 20.449 & 589.8 & 2 \text {-methylpentene-1 } \\ 20.532 & 590.6 & \text { hexene-1 } \\ 21.440 & 600.0 & \text { n-hexane } \\ 21.643 & 602.6 & \text { c-hexene-3 } \\ 21.699 & 603.3 & \text { ? } \\ & & \end{array}$

File: 431-2.DHA

$\begin{array}{rrr}\text { Wt } & \text { VOl\% } & \text { Mo1\% } \\ 0.002 & 0.004 & 0.004 \\ 0.735 & 1.246 & 1.401 \\ 14.236 & 16.394 & 18.504 \\ 0.016 & 0.018 & 0.021 \\ 0.008 & 0.010 & 0.011 \\ 10.835 & 11.208 & 10.685 \\ 10.570 & 10.237 & 10.798 \\ 30.807 & 30.678 & 30.380 \\ 5.104 & 4.869 & 5.214 \\ 0.225 & 0.220 & 0.179 \\ 0.006 & 0.006 & 0.005 \\ 5.641 & 5.233 & 5.762 \\ 0.001 & 0.001 & 0.001 \\ 0.006 & 0.004 & 0.007 \\ 0.403 & 0.371 & 0.330 \\ 10.750 & 10.000 & 8.540 \\ 0.440 & 0.396 & 0.360 \\ 0.005 & 0.003 & 0.004 \\ 0.006 & 0.004 & 0.006 \\ 0.170 & 0.151 & 0.139 \\ 6.430 & 5.918 & 5.108 \\ 0.006 & 0.005 & 0.005 \\ 0.281 & 0.250 & 0.230 \\ 0.001 & 0.001 & 0.001 \\ 0.138 & 0.121 & 0.113 \\ 0.159 & 0.138 & 0.130 \\ 0.008 & 0.007 & 0.005 \\ 0.066 & 0.059 & 0.054 \\ 0.046 & 0.034 & 0.038 \\ 0.021 & 0.018 & 0.014 \\ 0.008 & 0.007 & 0.006 \\ 0.429 & 0.332 & 0.351 \\ 0.061 & 0.053 & 0.040 \\ 0.003 & 0.002 & 0.002 \\ 0.006 & 0.005 & 0.004 \\ 0.831 & 0.733 & 0.553 \\ 0.018 & 0.015 & 0.012 \\ 0.233 & 0.202 & 0.155 \\ 0.014 & 0.012 & 0.010 \\ 0.017 & 0.014 & 0.011 \\ 0.478 & 0.418 & 0.318 \\ 0.007 & 0.006 & 0.005 \\ 0.002 & 0.002 & 0.001\end{array}$

p. 1 
Components Listed in Chromatographic order

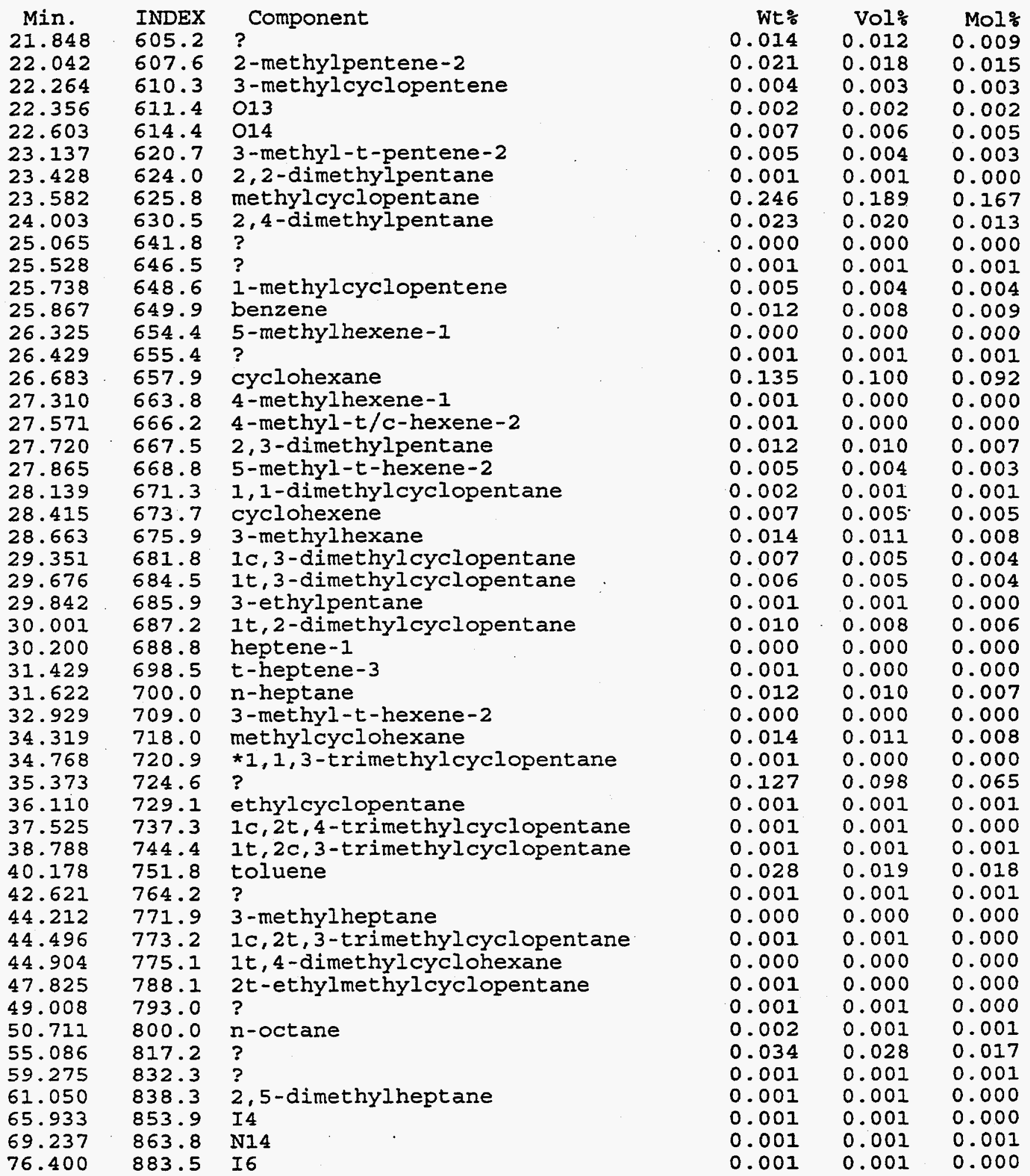

File: $431-2$. DHA

Sample: $97-431-2$

p. 2 
CALEB BRETT HOUSTON

TID: 97-000431-0-HOUS-002-00

CID: CONSOLINC

SID: HTI PBO5-22,23,24,25 CRUDE

OIL/IBP-70 F

NID : 58159

Date: 10-JAN-1997

Analyzed: 1/29/97 9:49 AM

Reported: 01-29-1997 11:45:10 Normalized to $100.00 \%$

Comments :

Composite Report

Totals by Group Type \& Carbon Number (in Weight Percent)

Paraffins: I-paraffins: Aromatics: Naphthenes: Olefins: Total:

$\begin{array}{lrrrrrr}\text { C1: } & 0.000 & 0.000 & 0.000 & 0.000 & 0.000 & 0.000 \\ \text { C2: } & 0.735 & 0.000 & 0.000 & 0.000 & 0.002 & 0.737 \\ \text { C3: } & 14.236 & 0.000 & 0.000 & 0.000 & 0.000 & 14.236 \\ \text { C4: } & 30.807 & 10.835 & 0.000 & 0.000 & 21.315 & 62.957 \\ \text { C5: } & 6.430 & 10.975 & 0.000 & 0.429 & 1.711 & 19.545 \\ \text { C6: } & 0.478 & 1.132 & 0.012 & 0.381 & 0.139 & 2.142 \\ \text { C7: } & 0.012 & 0.050 & 0.028 & 0.041 & 0.008 & 0.138 \\ \text { C8: } & 0.002 & 0.000 & 0.000 & 0.005 & 0.000 & 0.007 \\ \text { C9: } & 0.000 & 0.002 & 0.000 & 0.001 & 0.000 & 0.004 \\ \text { C10: } & 0.000 & 0.000 & 0.000 & 0.000 & 0.000 & 0.000 \\ \text { C11: } & 0.000 & 0.000 & 0.000 & 0.000 & 0.000 & 0.000 \\ \text { C12: } & 0.000 & 0.000 & 0.000 & 0.000 & 0.000 & 0.000 \\ \text { C13: } & 0.000 & 0.000 & 0.000 & 0.000 & 0.000 & 0.000 \\ \text { C14: } & 0.000 & 0.000 & 0.000 & 0.000 & 0.000 & 0.000 \\ \text { Total: } 52.700 & 22.995 & 0.040 & 0.856 & 23.173 & 99.765 \\ \text { Oxygenates: } & 0.011 & \text { Total C14+: } & 0.000 & \text { Total Unknowns: } & 0.225 \\ \text { Oxys } & & & & & & \text { Grand Total: } 100.000\end{array}$

Molecular Weight and Relative Density Data

$\begin{array}{rrr}\text { Group: } & \text { Ave. Mw.: } & \text { Ave. Rel. Density: } \\ \text { C1: } & 0.000 & 0.000 \\ \text { C2: } & 30.064 & 0.340 \\ \text { C3: } & 44.097 & 0.501 \\ \text { C4: } & 57.425 & 0.583 \\ \text { C5: } & 71.919 & 0.626 \\ \text { C6: } & 85.615 & 0.676 \\ \text { C7: } & 97.749 & 0.737 \\ \text { C8: } & 112.832 & 0.745 \\ \text { C9: } & 127.536 & 0.744 \\ \text { C10: } & 0.000 & 0.000 \\ \text { C11: } & 0.000 & 0.000 \\ \text { C12: } & 0.000 & 0.000 \\ \text { C13: } & 0.000 & 0.000 \\ \text { C14: } & 0.000 & 0.000 \\ \text { Total Sample: } & 57.184 & 0.575\end{array}$

File: $431-2$. DHA 
TID: 97-000431-0-HOUS-002-00

CID: CONSOLINC

SID: HTI PBO5-22,23,24,25 CRUDE OIL/IBP-70 F

NID: 58159

Date: 10-JAN-1997

Analyzed: 1/29/97 9:49 AM

Reported: 01-29-1997 11:45:10 Normalized to $100.00 \%$

Comments :

Composite Report

Totals by Group Type \& Carbon Number (in Volume Percent)

Paraffins:

$\begin{array}{lr}\text { C1: } & 0.000 \\ \text { C2: } & 1.246 \\ \text { C3: } & 16.394 \\ \text { C4: } & 30.678 \\ \text { C5: } & 5.918 \\ \text { C6: } & 0.418 \\ \text { C7: } & 0.010 \\ \text { C8: } & 0.001 \\ \text { C9: } & 0.000 \\ \text { C10: } & 0.000 \\ \text { C11 } & 0.000 \\ \text { C12: } & 0.000 \\ \text { C13: } & 0.000 \\ \text { C14: } & 0.000\end{array}$

Total: 54.665

Oxygenates: $0.008 \quad$ Total Cl4t: 0.000

\begin{abstract}
I-paraffin
0.000
0.000

0.000

11.208

10.220

0.995

0.042

0.000

0.002

0.000

0.000

0.000

0.000

0.000
\end{abstract}

22.467

\begin{abstract}
0.000
0.000

0.000

0.000

0.000

0.008

0.019

0.000

0.000

0.000

0.000

0.000

0.000

0.000

0.027
\end{abstract}

Olefins:

0.000

0.000

0.000

0.000

0.332

0.289

0.031

0.003

0.001

0.000

0.000

0.000

0.000

0.000

0.656
0.000

0.004

0.000

20.339

1.526

0.116

0.006

0.000

0.000

0.000

0.000

0.000

0.000

0.000

21.990
Total:

0.000

1. 250

16.394

62.224

17.996

1.826

0.108

0.005

0.003

0.000

0.000

0.000

0.000

0.000

99.806

Total Unknowns:

0.187

Grand Total: 100.000

(in Mole Percent)

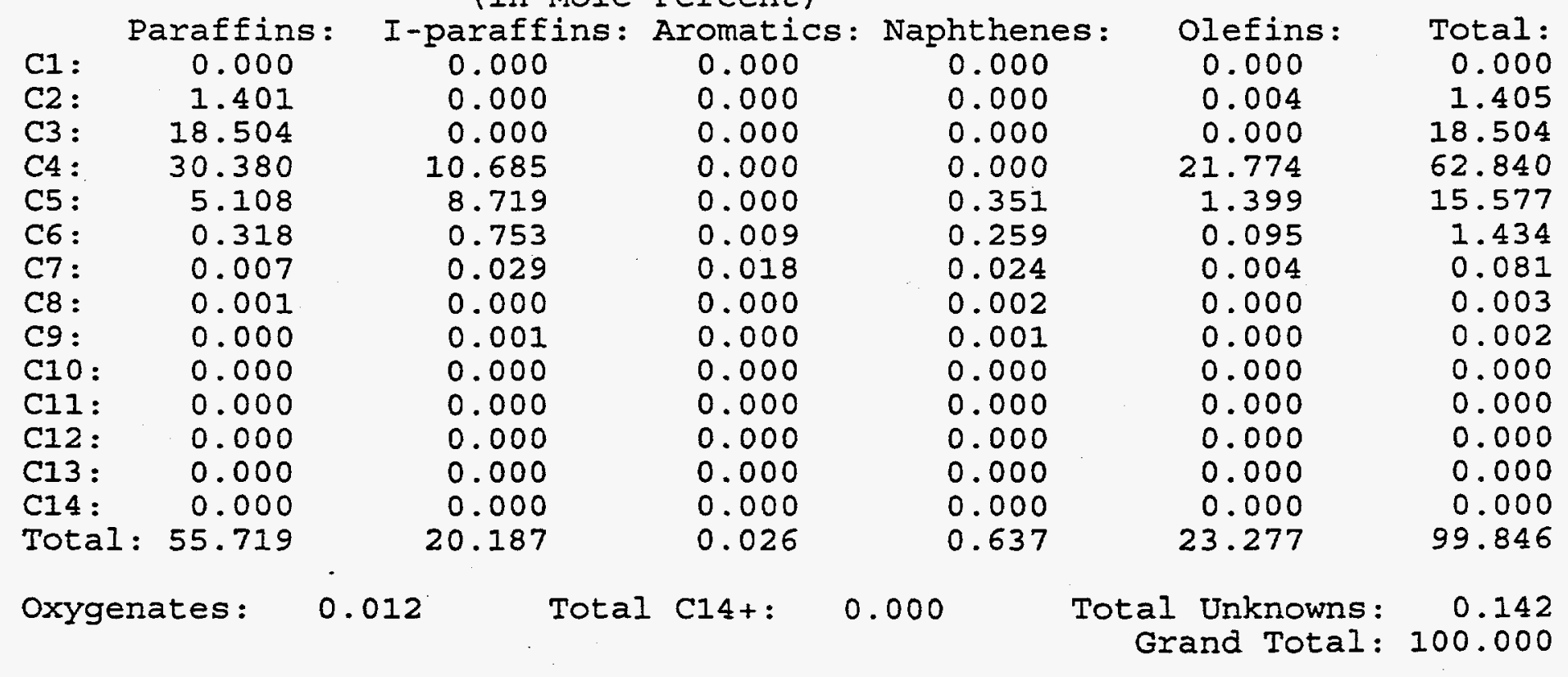

File: $431-2$. DHA 
TID: 97-000431-0-HOUS-002-00

CID: CONSOLINC

SID: HTI PBO5-22, 23, 24,25 CRUDE OIL/IBP-70 F

NID: 58159

Date: $10-$ JAN-1997

Analyzed: 1/29/97 9:49 AM

Reported: 01-29-1997 11:45:10 Normalized to $100.00 \%$

Comments :

Boiling Point Distribution Data

\begin{tabular}{rrrrrr} 
& & Wt. Percent Off: & \multicolumn{2}{c}{ Vol. Percent Off: } \\
IBP & deg.C.: & deg.F.: & deg.C.: & deg.F.: \\
(0.5\%) & -88.60 & -127.48 & -88.60 & -127.48 \\
$5.0 \%$ & -42.04 & -43.67 & -42.04 & -43.67 \\
$10.0 \%$ & -42.04 & -43.67 & -42.04 & -43.67 \\
$15.0 \%$ & -11.72 & 10.90 & -42.04 & -43.67 \\
$20.0 \%$ & -11.72 & 10.90 & -11.72 & 10.90 \\
$25.0 \%$ & -11.72 & 10.90 & -11.72 & 10.90 \\
$30.0 \%$ & -6.25 & 20.75 & -6.25 & 20.75 \\
$35.0 \%$ & -6.25 & 20.75 & -6.25 & 20.75 \\
$40.0 \%$ & -0.50 & 31.10 & -0.50 & 31.10 \\
$45.0 \%$ & -0.50 & 31.10 & -0.50 & 31.10 \\
$50.0 \%$ & -0.50 & 31.10 & -0.50 & 31.10 \\
$55.0 \%$ & -0.50 & 31.10 & -0.50 & 31.10 \\
$60.0 \%$ & -0.50 & 31.10 & -0.50 & 31.10 \\
$65.0 \%$ & -0.50 & 31.10 & -0.50 & 31.10 \\
$70.0 \%$ & 0.88 & 33.58 & 0.88 & 33.58 \\
$75.0 \%$ & 3.72 & 38.70 & 3.72 & 38.7 .0 \\
$80.0 \%$ & 27.84 & 82.11 & 9.50 & 49.10 \\
$85.0 \%$ & 27.84 & 82.11 & 27.84 & 82.11 \\
$90.0 \%$ & 36.06 & 96.91 & 27.84 & 82.11 \\
$95.0 \%$ & 36.06 & 96.91 & 36.06 & 96.91 \\
FBP (99. & 71.80 & 161.24 & 71.80 & 161.24
\end{tabular}

Research Octane Number $=108.61$

(Calculated from Individual Component Values)

$\begin{array}{lc}\text { Contribution to Total by: } \\ \text { Paraffins: } & 57.15 \\ \text { Iso-paraffins: } & 24.97 \\ \text { Aromatics: } & 0.05 \\ \text { Naphthenes: } & 0.75 \\ \text { Olefins: } & 25.53 \\ \text { Oxygenates: } & 0.01\end{array}$

File: $431-2$. DHA 


\section{Intertek Testing Services}

Caleb Brett

\section{WinAssay '95}

Version 1.00

Final Reports

\begin{tabular}{|ll|}
\hline Client Name: & Consol Inc. \\
Sample ID: & $\underline{\text { HTI PB-05-22,23,24,25 }}$ \\
Laboratory ID: & $\underline{97-000431}$ \\
Date: & $\underline{02 / 14 / 97}$ \\
Operator: & $\underline{\text { Robert Kelly }}$ \\
\hline
\end{tabular}




\begin{tabular}{|c|c|c|c|c|c|c|c|c|c|c|}
\hline \multicolumn{2}{|c|}{$\begin{array}{l}\text { Prepared For: } \\
\text { Sample ID: } \\
\text { Date: }\end{array}$} & \multicolumn{9}{|c|}{$\begin{array}{l}\text { Consol Inc. } \\
\text { HTI PB-05-22,23,24,25 } \\
\text { 02/14/97 }\end{array}$} \\
\hline $\begin{array}{r}\text { Cut Temp } \\
\text { To }\end{array}$ & Degrees F & $\begin{array}{l}\text { DUMP } \\
\text { WT(g) }\end{array}$ & $\begin{array}{l}\text { Specific } \\
\text { Gravity }\end{array}$ & MLS & $\begin{array}{c}\text { LIQ } \\
\text { YOL\% }\end{array}$ & $\begin{array}{c}\text { CUM. LIQ } \\
\text { voL\% }\end{array}$ & WT $\%$ & $\begin{array}{l}\text { CUM } \\
\text { WT } \%\end{array}$ & $\begin{array}{c}\text { API } \\
\text { GRAVITY } \\
\end{array}$ & $\begin{array}{c}\text { MID } \\
\text { LIQ VOL } \%\end{array}$ \\
\hline \multicolumn{11}{|c|}{ ASTM D2892 Distillation Yields } \\
\hline IBP & 70 & 82.40 & 0.5826 & 141.43 & 1.19 & 1.19 & 0.81 & 0.81 & 111.38 & 0.60 \\
\hline 70 & 180 & 616.20 & 0.6854 & 899.04 & 7.57 & 8.76 & 6.07 & 6.88 & 74.95 & 4.98 \\
\hline 180 & 350 & 2743.70 & 0.7862 & 3489.82 & 29.38 & 38.14 & 27.01 & 33.89 & 48.48 & 23.45 \\
\hline 350 & 400 & 1014.80 & 0.8565 & 1184.82 & 9.98 & 48.12 & 9.99 & 43.88 & 33.71 & 43.13 \\
\hline 400 & 550 & 3320.10 & 0.8932 & 3717.08 & 31.30 & 79.42 & 32.68 & 76.56 & 26.92 & 63.77 \\
\hline 550 & 650 & 1526.30 & 0.9355 & 1631.53 & 13.74 & 93.16 & 15.03 & 91.59 & 19.76 & 86.29 \\
\hline $650+$ & & 854.50 & 0.9923 & 861.13 & 7.25 & 100.41 & 8.41 & 100.00 & 11.10 & \\
\hline
\end{tabular}


WinAssay '95 Quality Control Applications

Cum. Mid Vol\% v. API Gravity

HTI PB-05-22,23,24,25

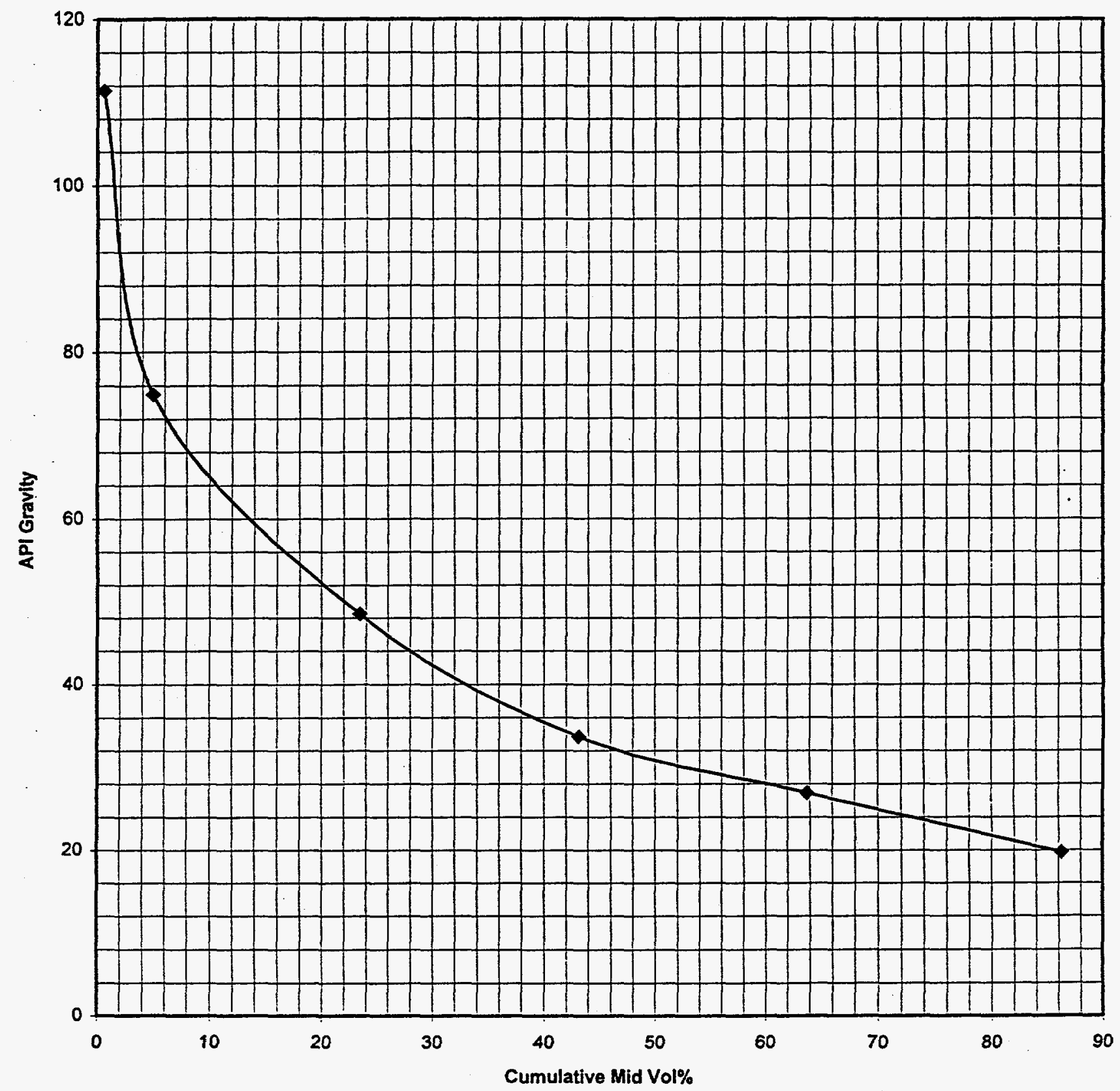

API CURVE Chart 1 


WinAssay '95
True Boiling Point Curve
Vaporline Temperature v. Cumulative Wt\% Yield

Sample ID

HTI PB-05-22,23,24,25

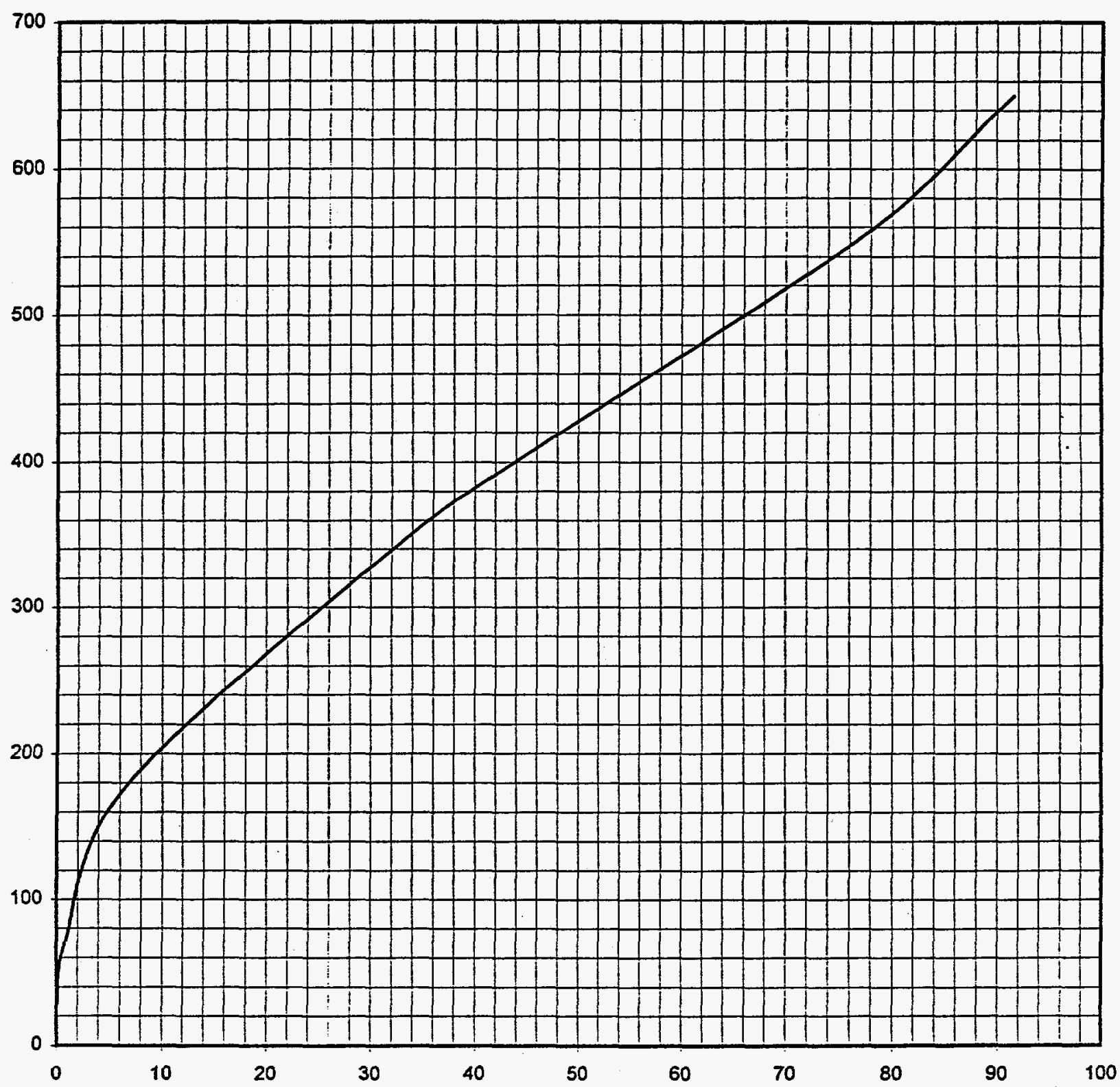




\section{WinAssay '95 \\ True Boiling Point Curve vs Cumulative Vol\% Yield}

Sample ID

HTI PB-05-22,23,24,25

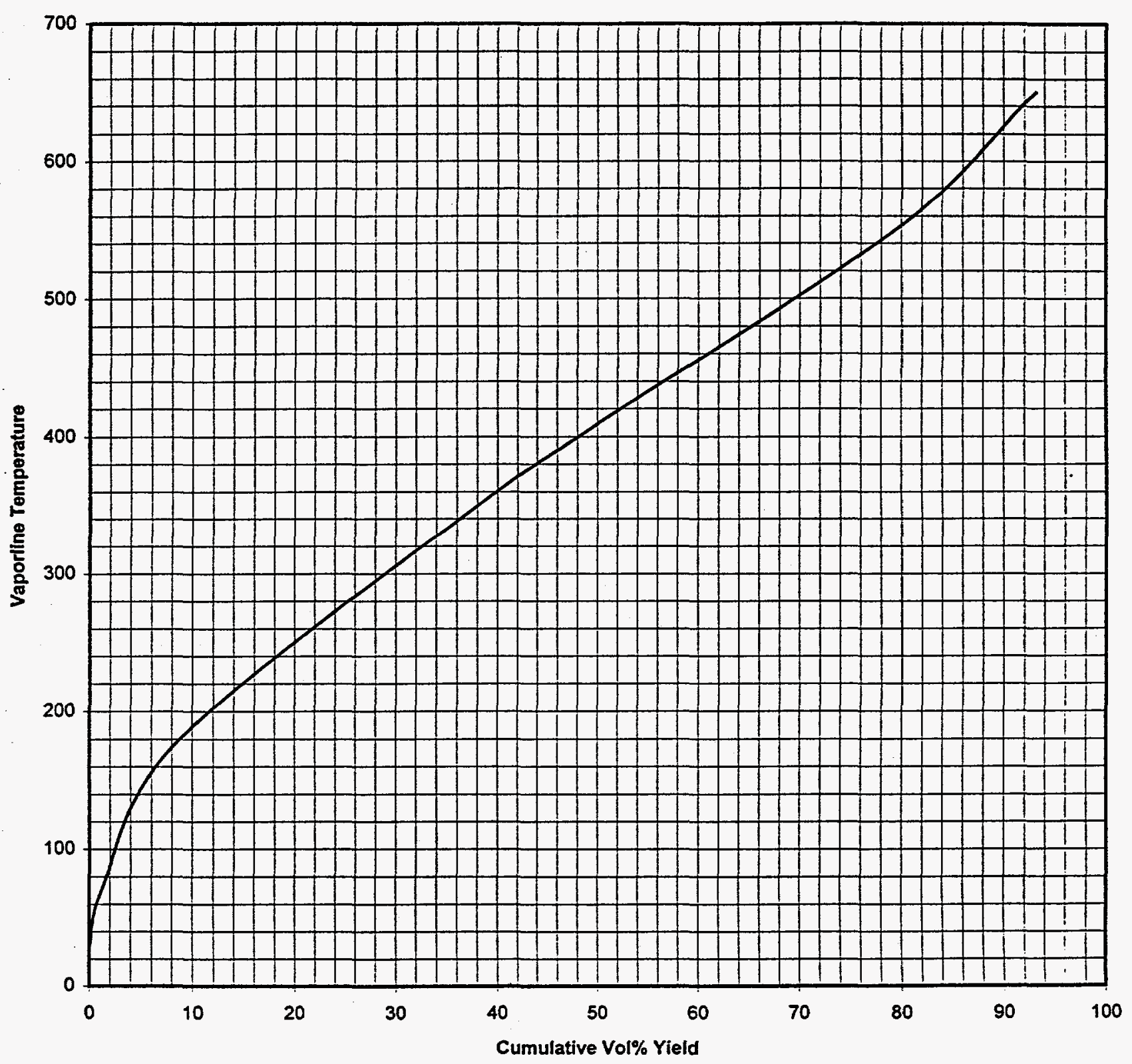


ITS- Caleb Brett Distillation Group

ASTM D2892/D5236 CHARGE INFORMATION

Lab ID:

Client Name:

97-000431

Consol Inc.

Sample ID:

HTI PB-05-22,23,24,25

Date:

$02 / 14 / 97$

Operator: Robert Kelly

$\stackrel{\vec{\omega}}{\omega}$

Charge Mass D2892(g):

Charge S.G D2892 (60/60F):

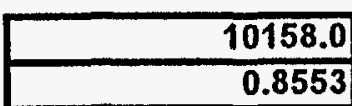

0.8553

0.0

0.0000

Charge S.G. D5236 (60/60F):

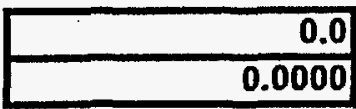

Water Weight Removed (g):

Initial Vapor Temp:

Whole Crude Sulfur Wt\%:

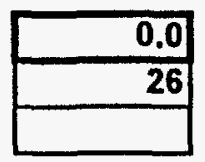


WinAssay '95

ASTM TBP And Potstill Distillation

Quality Control Report

Sample ID: $\quad$ HTI PB-05-22,23,24,25

Lab ID: $\quad$ 97-000431

$\begin{array}{lll}\text { Client: } & \text { Consol Inc. } & \text { Date: }\end{array}$

\section{Material Balance Parameters:}

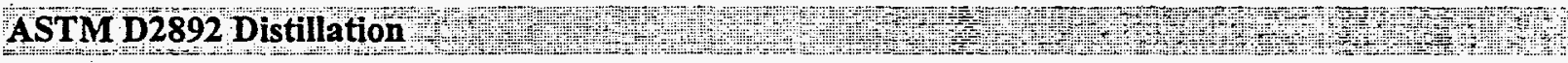

D2892 Material Balance:

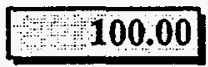

Passes Material Balance Per D2892

API Balance Parameters:

D2892 Measured API:

$$
\begin{array}{|r|}
\hline 33.9 \\
\hline 34.6 \\
\hline
\end{array}
$$

API Delta (Meas-Calc): $\quad$ Passes API Test

Note: Review the API vs Mid-Vol\% Plot For Outliers along the curve. Points lying off the curvature should be reviewed for accuracy in density determination. 


\section{Intertek Testing Services Caleb Brett}

\section{Temperature Readings At 10\% (Volume) Increments}

Date: February 14,1997

Sample Description: HTI PB-05-22,23,24,25

**NOTE** The following data was estimated during the ASTM D2892 Distillation Process and actual $10 \%$ readings may vary, refer to ASTM D2892 Distillation Summary Report for more precise data.

\begin{tabular}{cc} 
\% Volume & Temperature (Deg.F) \\
\cline { 2 - 2 } 10 & 187 \\
20 & 249 \\
30 & 310 \\
40 & 357 \\
50 & 406 \\
60 & 455 \\
70 & 501 \\
80 & 551 \\
90 & 624
\end{tabular}




\section{APPENDIX 2}

CALEB BRETT REPORT ON FRACTIONAL DISTILLATION OF NET PRODUCTS OF HTI RUN POC-1 


\section{TTS Intertek Testing Services Caleb Brett}

9809 Rowlett Road
Houston, TX 77075
Phone: (713) $844-3200$
Fax: (713) $844-3330$

Your Ref: PO\# 01-001-034304

Date: $15-A P R-1997$

\section{Laboratory Report No. 97-000432-0-HOUS; 1}

Consol, Inc.

Research \& Development

4000 Brownsville Road

Library, PA 15129-9566

For the Attention of R.A. Winschel

SAMPLE DETAILS:

SOURCE

:

2 cut(s) from one sample received on 10-JAN-1997

Consol, Ine.

CUSTOMER PRODUCT

DESCRIPTION

\author{
Consol, Inc.
}




\section{TTS Intertek Testing Services}

Caleb Brett

Laboratory Report No. 97-000432-0-HoUs; 1 - Page 2 of 2

Sample ID

97-000432-0-HOUs-001-00

Test

Initial Boiling Point

e 58 Evaporated

a $10 \%$ Evaporated

e 208 Evaporated

e 308 Evaporated

e $40 \%$ Evaporated

e 508 Evaporated

e 608 Evaporated

a 708 Evaporated

e 808 Evaporated

e 908 Evaporated

Final Boiling Point

Recovery

Residue

Ioss

Boiling Range Distribution
Customer Product Description

"HTI POC 1 CRUDE OIL"

Sample As Received

$\begin{array}{lrr} & \text { Method } & \text { 001-00 } \\ \text { Deg. F } & \text { D86 } & 174 \\ \text { Deg. F } & & 220 \\ \text { Deg. F } & & 242 \\ \text { Deg. F } & & 300 \\ \text { Deg. F } & & 374 \\ \text { Deg. F } & 440 \\ \text { Deg. F } & & 483 \\ \text { Deg. F } & & 520 \\ \text { Deg. F } & & 558 \\ \text { Deg. F } & 590 \\ \text { Deg. F } & & 650 \\ \text { Deg. F } & & 655 \\ \text { Vol. } 8 & 93.0 \\ \text { Vol. } 8 & & 6.5 \\ \text { Vol. } 8 & & 0.5 \\ & \text { D2887 } & \text { See Attached }\end{array}$

Sample ID

Customer Product Description

"HTI POC 1 CRUDE OIL"

IBP-70 Deg. $F$

Test

Method

$\underline{002-00}$

Detailed Hydrocarbon Analysis

GC

See Attached 
WinAssay '95

Version 1.00

Final Reports

\begin{tabular}{|ll|}
\hline Client Name: & $\underline{\text { Consol Inc. }}$ \\
Sample ID: & $\underline{\text { HTI POC 1 }}$ \\
Laboratory ID: & $\underline{97-000432}$ \\
Date: & $\underline{02 / 14 / 97}$ \\
Operator: & $\underline{\text { Robert Kelly }}$ \\
\hline
\end{tabular}




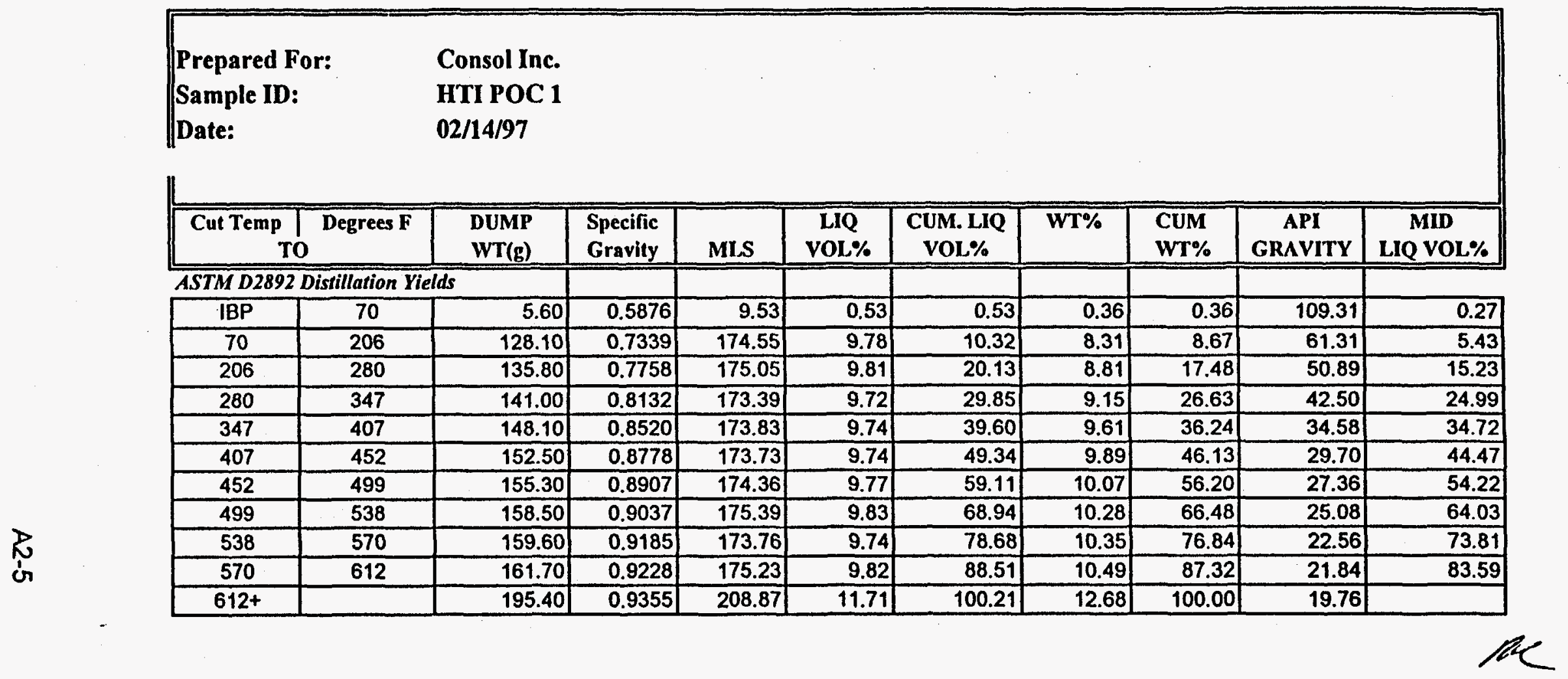


WinAssay '95 Quality Control Applications

Cum. Mid Vol\% v. API Gravity

HTI POC 1

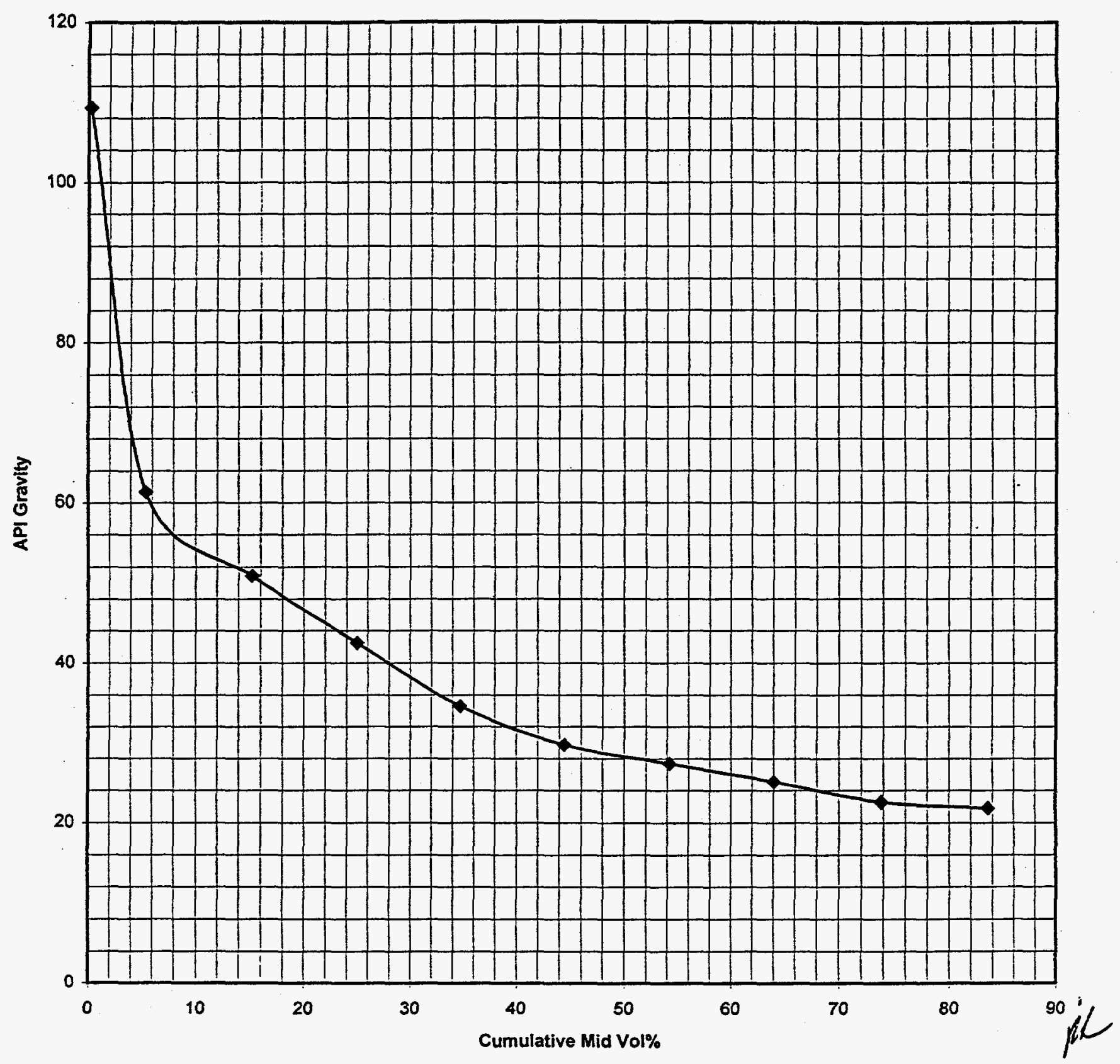




WinAssay '95
True Boiling Point Curve
Vaporline Temperature v. Cumulative Wt\% Yield

Sample ID

HTI POC 1

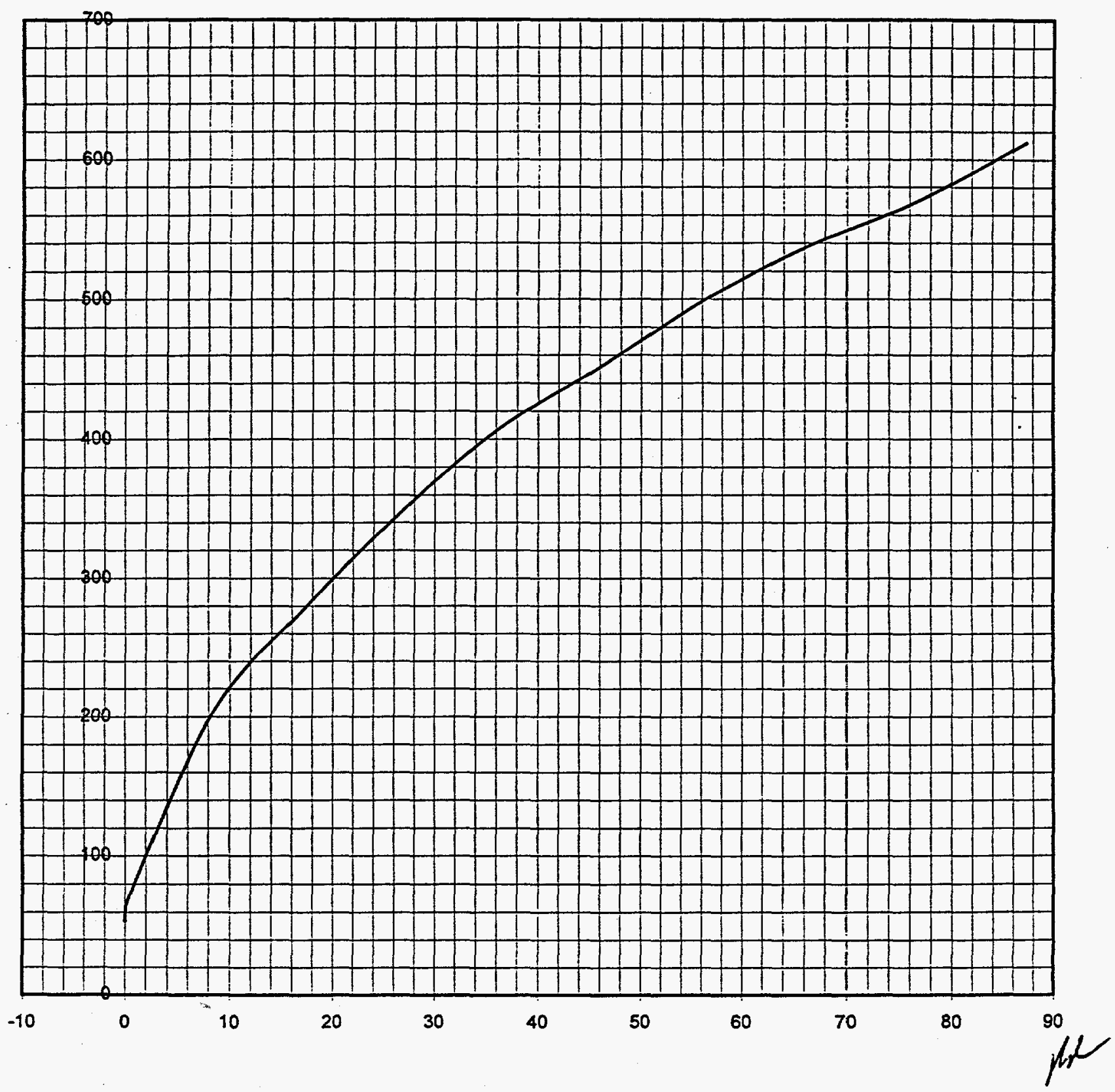




\section{WinAssay ' 95 \\ True Boiling Point Curve vs Cumulative Vol\% Yield}

Sample ID

HTI POC 1

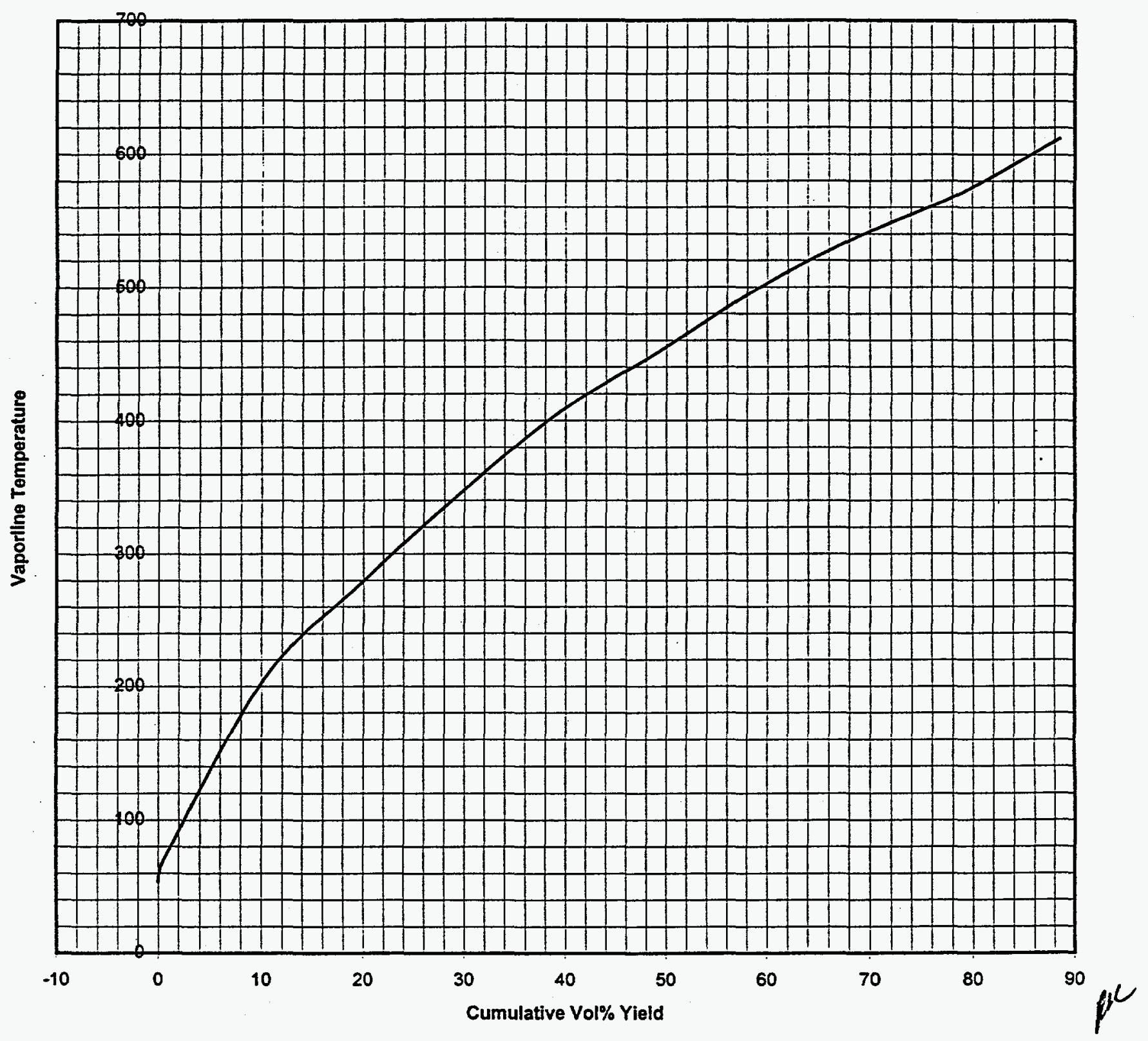


ITS- Caleb Brett Distillation Group

ASTM D2892/D5236 CHARGE INFORMATION

Lab ID:

Client Name:

Sample ID:

97-000432

Consol Inc.

HTI POC 1

Date:

$02 / 14 / 97$

Operator: Robert Kelly

R

$\dot{6}$

Charge Mass D2892(g):

Charge S.G D2892 (60/60F):

$\begin{array}{r}\hline 1541.6 \\ \hline 0.8642 \\ \hline\end{array}$

Water Weight Removed (g):

Initial Vapor Temp:

Whole Crude Sulfur Wt\%:

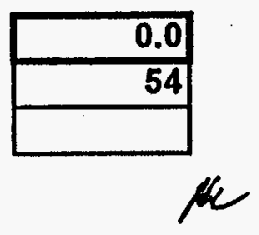

Charge Mass D5236(g)

0.0

0.0000

Charge S.G. D5236 (60/60F):

$\begin{array}{r}0.0 \\ \hline 0.0000 \\ \hline\end{array}$


WinAssay ' 95

ASTM TBP And Potstill Distillation

Quality Control Report

Sample D: $\quad$ HTI POC 1

Lab ID: $\quad 97-000432$

Client: Consol Inc. Date: 02/14/97

\section{Material Balance Parameters:}

ASTM D282 Distillation

D2892 Material Balance: $\quad$ 100.00 $\quad$ Passes Material Balance Per D2892

\section{API Balance Parameters:}

D2892 Measured API:

D2892 Calc API:

\begin{tabular}{|r|}
\hline 332.2 \\
\hline $3+32.6$ \\
\hline
\end{tabular}

API Delta (Meas-Calc): $\quad$ Passes API Test

Note: Review the API vs Mid-Vol\% Plot For Outliers along the curve. Points lying off the curvature should be reviewed for accuracy in density determination.<smiles>CC1CCCC1</smiles> 
CALEB BRET' HUUSTION

TIE: 97-000432-0-HOUS-001-00

APR 01, $1997-16: 45: 26$

SIMDIS EXPERT V5.0

CID: CONSOLINC

SID: HTI POC 1 CRUDE OIL/AS RECEIVED

NID: 58261

Date: 10-JAN-1997

Page 3

Boiling Point Distribution

CRUDE EXT_STD HI-TEMP

ASTM D2887

\begin{tabular}{|c|c|c|c|c|c|c|c|c|}
\hline$\%$ off & $B P(F)$ & $B P(C)$ & $\%$ Off & BP(F) & $\mathrm{BP}(\mathrm{C})$ & $\%$ off & $\mathrm{BP}(\mathrm{E})$ & $B P(C)$ \\
\hline IBP & 65.0 & 18.3 & 40 & 426.6 & 219.2 & 80 & 580.5 & 304.7 \\
\hline 1 & 99.0 & 37.2 & 41 & 432.3 & 222.4 & 81 & 583.9 & 306.6 \\
\hline 2 & 155.4 & 68.6 & 42 & 439.5 & 226.4 & 82 & 587.6 & 308.7 \\
\hline 3 & 169.6 & 76.4 & 43 & 443.7 & 228.7 & 83 & 591.1 & 310.6 \\
\hline 4 & 183.2 & 84.0 & 44 & 446.8 & 230.4 & 84 & 594.5 & 312.5 \\
\hline 5 & 194.4 & 90.2 & 45 & 451.5 & 233.1 & 85 & 598.5 & 314.7 \\
\hline 6 & 204.2 & 95.7 & 46 & 455.6 & 235.3 & 86 & 601.8 & 316.6 \\
\hline 7 & 211.3 & 99.6 & 47 & 460.2 & 237.9 & 87 & 605.7 & 318.7 \\
\hline 8 & 215.9 & 102.2 & 48 & 464.0 & 240.0 & 88 & 609.9 & 321.1 \\
\hline 9 & 220.8 & 104.9 & 49 & 468.6 & 242.6 & 89 & 614.2 & 323.4 \\
\hline 10 & 226.7 & 108.2 & 50 & 472.9 & 244.9 & 90 & 619.0 & 326.1 \\
\hline 11 & 234.6 & 112.6 & 51 & 477.6 & 247.6 & 91 & 623.9 & 328.8 \\
\hline 12 & 244.4 & 118.0 & 52 & 482.7 & 250.4 & 92 & 627.9 & 331.1 \\
\hline 13 & 253.4 & 123.0 & 53 & 487.3 & 252.9 & 93 & 633.3 & 334.1 \\
\hline 14 & 262.6 & 128.1 & 54 & 490.1 & 254.5 & 94 & 639.4 & 337.4 \\
\hline 15 & 268.6 & 131.4 & 55 & 493.7 & 256.5 & 95 & 645.8 & 341.0 \\
\hline 16 & 274.7 & 134.8 & 56 & 497.0 & 258.3 & 96 & 652.2 & 344.6 \\
\hline 17 & 285.5 & 140.8 & 57 & 501.4 & 260.8 & 97 & 659.3 & 348.5 \\
\hline 18 & 293.2 & 145.1 & 58 & 505.0 & 262.8 & 98 & 667.6 & 353.1 \\
\hline 19 & 301.5 & 149.7 & 59 & 508.6 & 264.8 & 99 & 677.5 & 358.6 \\
\hline 20 & 308.6 & 153.7 & 60 & 512.5 & 266.9 & & & \\
\hline 21 & 313.5 & 156.4 & 61 & 516.1 & 268.9 & & & \\
\hline 22 & 320.5 & 160.3 & 62 & 519.6 & 270.9 & & & \\
\hline 23 & 330.3 & 165.7 & 63 & 523.2 & 272.9 & & & \\
\hline 24 & 334.8 & 168.2 & 64 & 527.2 & 275.1 & & & \\
\hline 25 & 340.6 & 171.4 & 65 & 531.0 & 277.2 & & & \\
\hline 26 & 347.6 & 175.3 & 66 & 534.6 & 279.2 & & & \\
\hline 27 & 354.9 & 179.4 & 67 & 538.0 & 281.1 & & & \\
\hline 28 & 359.0 & 181.7 & 68 & 541.7 & 283.2 & & & \\
\hline 29 & 365.0 & 185.0 & 69 & 545.8 & 285.4 & & & \\
\hline 30 & 372.0 & 188.9 & 70 & 548.3 & 286.8 & & & \\
\hline 31 & 378.0 & 192.2 & 71 & 550.6 & 288.1 & & & \\
\hline 32 & 383.4 & 195.2 & 72 & 553.3 & 289.6 & & & \\
\hline 33 & 389.9 & 198.8 & 73 & 557.3 & 291.8 & & & \\
\hline 34 & 397.5 & 203.1 & 74 & 561.1 & 293.9 & & & \\
\hline 35 & 402.5 & 205.8 & 75 & 564.0 & 295.6 & & & \\
\hline 36 & 405.6 & 207.6 & 76 & 567.9 & 297.7 & & & \\
\hline 37 & 410.4 & 210.2 & 77 & 572.2 & 300.1 & & & \\
\hline 38 & 416.5 & 213.6 & 78 & 575.2 & 301.8 & & & \\
\hline 39 & 421.6 & 216.4 & 79 & 577.7 & 303.2 & & & \\
\hline
\end{tabular}

Start Time: 0.1 minutes

End Time: 23.1 minutes

Area: 346915328.0

Slice Width: $0.66 \mathrm{sec}$
Sample Offset: 17520.0

Baseline Offset: 18314.0

Calibration File: 0331 hrt

Calibration Date: 04/04/97
Residue: -99.0 at 859.2 F (459.5 C)

Concentration: 0.019435

Standard File: rgo0325h

Standard Date: 03/27/97 
CALEB BRETI HOUSTON

TID: 97-000432-0-HOUS-001-00

CID: CONSOLINC

SID: HTI POC 1 CRUDE OIL/AS RECEIVED

NID: 58261

Date: 10-JAN-1997

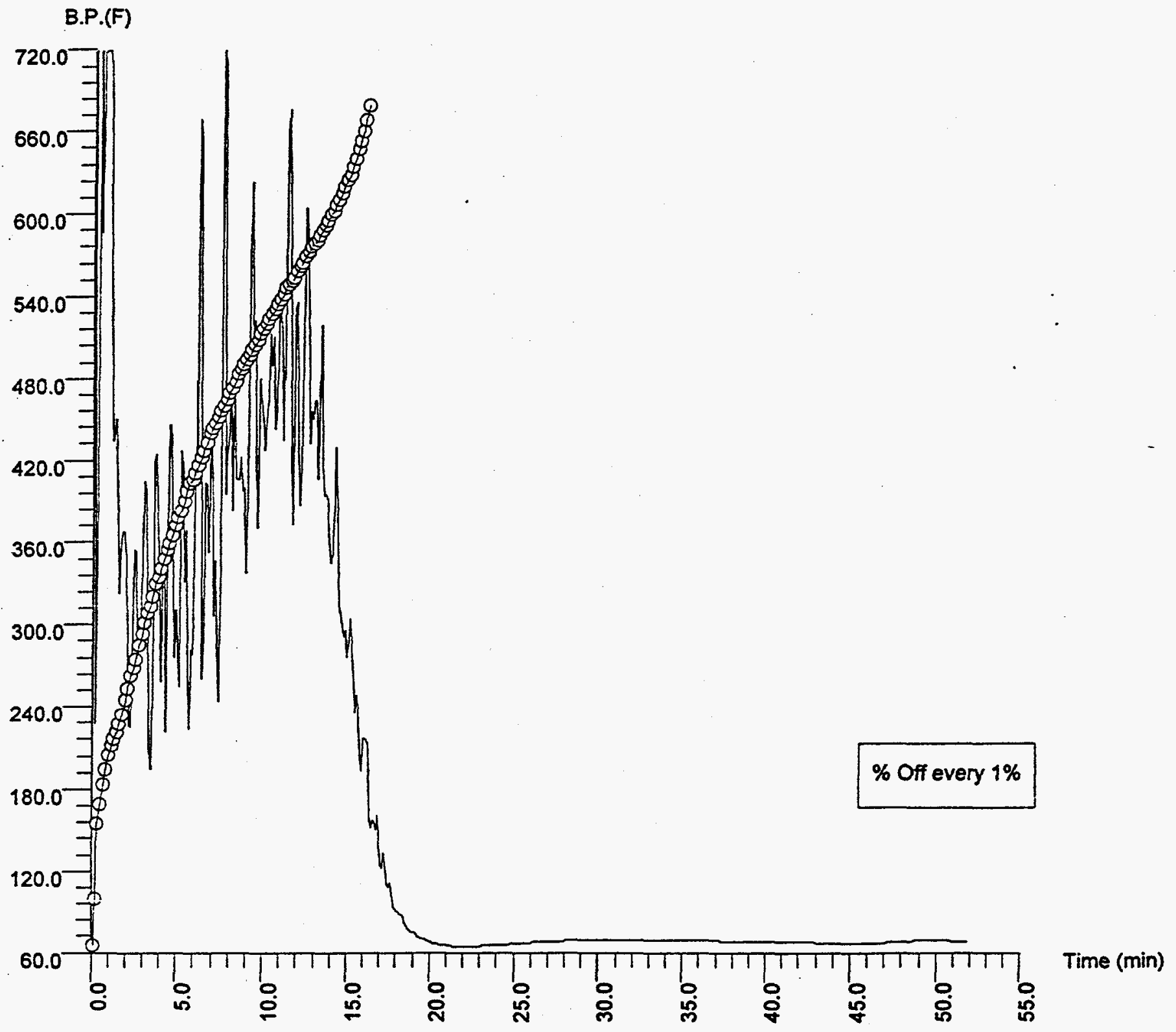


CALEB BRETT HOUSTON

TID: 97-000432-0-HOUS-001-00

CID: CONSOLINC

SID: HTI POC 1 CRUDE OIL/AS RECEIVED

NID: 58261

Date: $10-J A N-1997$

APR 01, 1997 - 16:45:26

SIMDIS EXPERT V5.0

Page 1

Calibration Plot CRUDE EXT_STD HI-TEMP

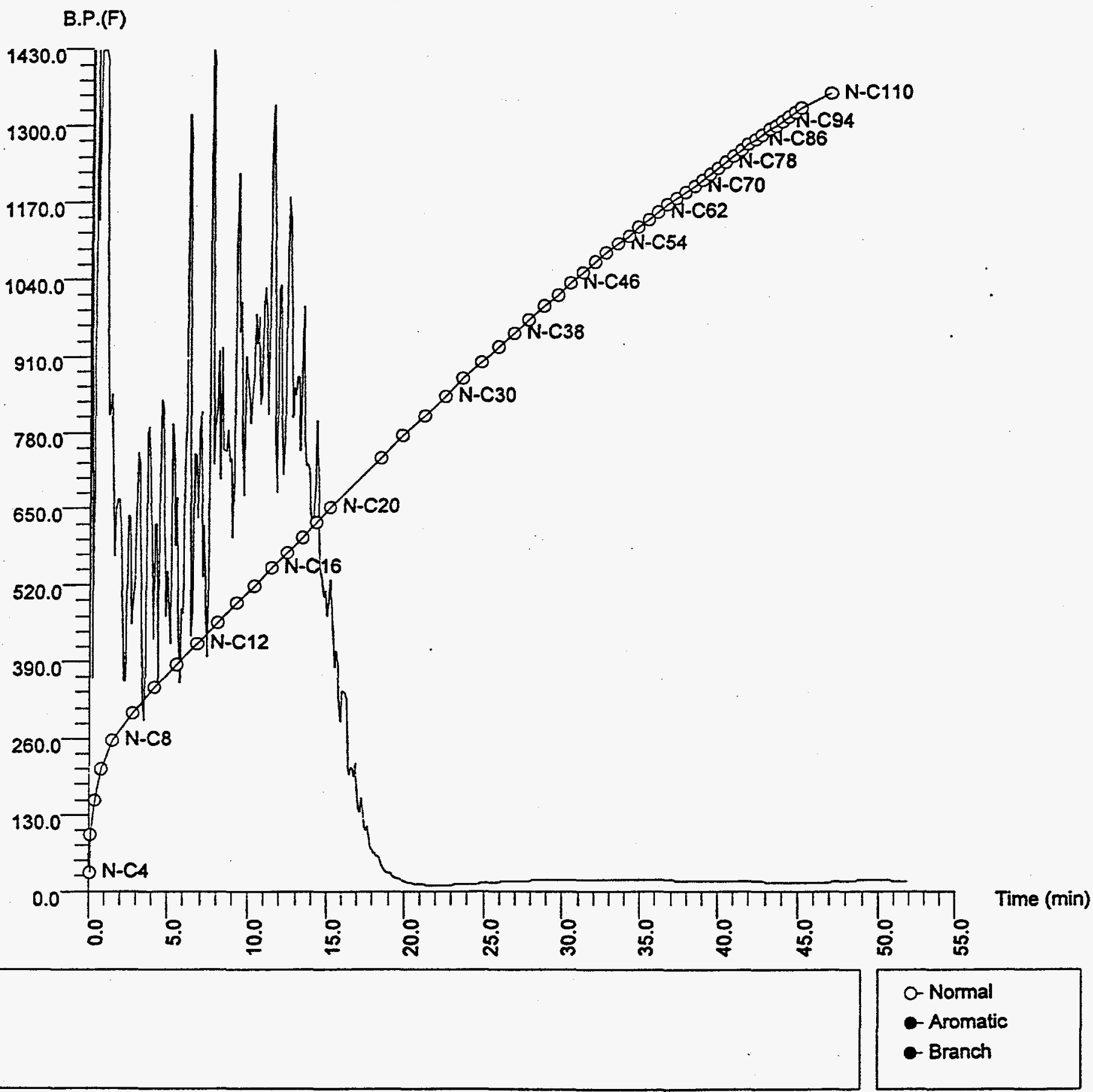




\begin{tabular}{|c|c|c|c|c|c|c|c|c|c|c|c|c|c|}
\hline \multicolumn{2}{|c|}{ Standard Practice for } & & & SAMPLE : & 97.432 .2 & & & & & & & & \\
\hline \multicolumn{14}{|c|}{ interconversion of Analysis of C5 and Lighter Hydrocarbons to } \\
\hline \multicolumn{14}{|c|}{\begin{tabular}{|l|l} 
Gas-Volume, Liquid-Volume, or Weight Basis & \\
\end{tabular}} \\
\hline \multirow{2}{*}{\multicolumn{2}{|c|}{ ASTM $02421-89$}} & & & & & & & & & & & & \\
\hline & & \multicolumn{4}{|c|}{ Conversion of Weight $\%$ to Gas or Liquid Volume \% } & & & Gas Vol\% & Gas Vol\% & & & & \\
\hline & & Enter wt\% & & Liq.vol. $1 \mathrm{ml}$ & Rel.dens. & Liq vol \% & Liq Vol\% & (mole \%) & (mole \%) & Vapor Pr. & Vapor Pr. & Sp.gravity & \\
\hline & & result here & Mol, wt. & idl.gas $60 / 760$ & $60 / 60$ (vac) & Unnorm & Norm & Unnorm & Norm & psig _ & Tot.psig & Average & \\
\hline Methane & & 0 & 16.04 & 0.00226 & 0.3 & of & 0 & 0 & 0 & 4985.3 & 0 & 0 & Methane \\
\hline Ethane & & 0.03 & 30.07 & 0.003566 & 0.3562 & 0.084222 & 0.049493 & 0.000998 & 0.059822 & 700 & 0.34645 & 0.000176 & Ethane \\
\hline Acetylene & & 0 & 26.04 & 0.00263 & 0.418 & 0 & 0 & 0 & 0 & & 0 & 0 & Acetylene \\
\hline Ethylene & & 0 & 28.05 & 0.0032 & 0.37 & of & 0 & 0 & 0 & & 0 & 0 & Ethylene \\
\hline Propane & & 8.8 & 44.1 & 0.003675 & 0.507 & 17.357 & 10.19975 & 0.199546 & 11.96514 & 174 & 17.74756 & 0.051713 & Propane \\
\hline Propylene & & 0.21 & 42.08 & 0.003413 & 0.521 & 0.403071 & 0.236862 & 0.00499 & 0.299238 & 213 & 0.504517 & 0.001234 & Propylene \\
\hline Propadiene & & 0 & 40.06 & 0.00282 & 0.6 & 0 & 0 & 0 & 0 & & 0 & 0 & Propadiene \\
\hline \multicolumn{2}{|c|}{ Methylacetylene } & 0 & 40.06 & 0.00273 & 0.621 & 0 & 0 & 0 & 0 & & 0 & 0 & Methylacetyleno \\
\hline n-Butane & & 49.19 & 58.12 & 0.004205 & 0.584 & 84.22945 & 49.49697 & 0.846352 & 50.74872 & 37 & 18,31388 & 0.289062 & n-Butane \\
\hline Isobutane & & 9.59 & 58.12 & 0.004362 & 0.5629 & 17.03677 & 10.01157 & 0.165003 & 9.893885 & 58 & 5.806708 & 0.056355 & Isobutane \\
\hline 1.Butene & & 3.8 & 56.11 & 0.003944 & 0.6011 & 6.321743 & 3.714938 & 0.067724 & 4.060852 & 47.4 & 1.76088 & 0.02233 & 1-Butene \\
\hline \multicolumn{2}{|c|}{ trans-2-Butene } & 1.33 & 56.11 & 0.003887 & 0.61 & 2.180328 & 1.281258 & 0.023703 & 1.421298 & 35.24 & 0.451515 & 0.007816 & trans-2-Butene \\
\hline \multicolumn{2}{|c|}{ cis-2-Butene } & 0.98 & 56.11 & 0.00378 & 0.6272 & 1.5625 & 0.918195 & 0.017466 & 1.047272 & 31.25 & 0.286936 & 0.005759 & cis-2-Butene \\
\hline \multirow{2}{*}{\multicolumn{2}{|c|}{\begin{tabular}{|l|} 
|sobutylene \\
2. Butadiene
\end{tabular}}} & 0 & 56.11 & 0.003949 & 0.6004 & 0 & 0 & 0 & 0 & 48.94 & 0 & 0 & Isobutylene \\
\hline & & 0 & 54.09 & 0.00347 & 0.658 & 0 & 0 & 0 & 0 & 21.8 & 0 & 0 & 1,2-Butadiene \\
\hline \multicolumn{2}{|c|}{ 1.3-Butadiene } & 0.4 & 54.09 & 0.003643 & 0.6272 & 0.637755 & 0.374773 & 0.007395 & 0.443422 & 44.7 & 0.167524 & 0.002351 & 1.3-Butadiene \\
\hline \multicolumn{2}{|c|}{ Ethylacetylene } & 0 & 54.09 & 0.00328 & 0.696 & 0 & 0 & 0 & 0 & & 0 & 0 & Ethylacetylene \\
\hline n-Pentane & & 10.92 & 72.15 & 0.00483 & 0.6311 & 17.30312 & 10.16808 & 0.151351 & 9.075283 & 0.875 & 0.088971 & 0.064171 & n-Pentane \\
\hline Isopentane & & 10.53 & 72.15 & 0.004879 & 0.6247 & \begin{tabular}{|c|}
16.85609 \\
\end{tabular} & 9.905389 & 0.145946 & 8.751166 & 5.744 & 0.568966 & 0.061879 & Isopentane \\
\hline \multicolumn{2}{|c|}{ Neopentane } & 0.26 & 72.15 & 0.005108 & 0.5967 & 0.43573 & 0.256054 & 0.003604 & 0.216078 & 21.96 & 0.05623 & 0.001528 & Neopentane \\
\hline 1-Pentene & & 0.56 & 70.13 & 0.004589 & 0.6457 & 0.867276 & 0.50965 & 0.007985 & 0.478804 & 4.417 & 0.022511 & 0.003291 & 1-Pentene \\
\hline \multicolumn{2}{|c|}{ trans-2.Pentene } & 0.23 & 70.13 & 0.004537 & 0.653 & 0.352221 & 0.20698 & 0.00328 & 0.196652 & & 0 & 0.001352 & trans-2-Pentene \\
\hline \multicolumn{2}{|c|}{ cis-2.Pentene } & 0.1 & 70.13 & 0.004482 & 0.6611 & 0.151263 & 0.088889 & 0.001426 & 0.085501 & & 0 & 0.000588 & cis-2-Pentene \\
\hline \multicolumn{2}{|c|}{ 2-Methyl-1-Butene } & 0.3 & 70.13 & 0.004519 & 0.6557 & 0.457526 & 0.268863 & 0.004278 & 0.256502 & & 0 & 0.001763 & 3)-Methyl-1-8utene \\
\hline \multicolumn{2}{|c|}{ 3-Methyl-1-Butene } & 0.2 & 70.13 & 0.004684 & 0.6325 & 0.316206 & 0.185816 & 0.002852 & 0.171002 & & 0 & 0.001175 & 3-Methyl-1-Butene \\
\hline \multicolumn{2}{|c|}{ 2-Methyl-2-Butene } & 0.23 & 70.13 & 0.00447 & 0.663 & 0.346908 & 0.203859 & 0.00328 & 0.196652 & & 0 & 0.001352 & 2-Methyl-2-Butene \\
\hline \multicolumn{2}{|c|}{ Cyclopentane } & 0.74 & 70.13 & 0.003948 & 0.7505 & 0.986009 & 0.579423 & 0.010552 & 0.632706 & & 0 & 0.004349 & Cyclopentane \\
\hline Isoprene & & 0 & 68.12 & 0.004195 & 0.6861 & 0 & 0 & 0 & 0 & & of & 0 & Isoprene \\
\hline \multicolumn{2}{|c|}{ 1-trans-3-Pentadiene } & 0 & 68.12 & 0.004224 & 0.6815 & 0 & 0 & 0 & 0 & & 0 & 0 & 1-trans-3-Pentadiene \\
\hline \multicolumn{2}{|c|}{ 1-cis-Pentadiene } & 0 & 68.12 & 0.004133 & 0.6964 & 0 & 0 & 0 & 0 & & 0 & 0 & 1-cis-Pentadiene \\
\hline 1.2.Pentad & & 0 & 68.12 & 0.004125 & 0.6976 & 0 & 0 & 0 & 0 & & 0 & 0 & 1.2.Pentadiene \\
\hline $\mathrm{C} 6+$ & & 1.6 & 85 & & 0.7 & 2.285714 & 1.343187 & & & & & 0.009402 & $66+$ \\
\hline & & & & & & & & & & & & & \\
\hline Total & & 100 & & & & 170.1709 & 100 & 1.667731 & 100 & & 46.12265 & 0.587644 & \\
\hline & & & & & & & & & & & & & \\
\hline Calculated & lapor Pres & & 46.12265 & & & & & & & & & & \\
\hline & & & & & & & & & & & & & \\
\hline & & & 0.587644 & & & & & & & & & & \\
\hline
\end{tabular}




\section{CALEB BRETT HOUSTON}

TID: 97-000432-0-HOUS-002-00

CID: CONSOLINC

SID: HTI POC 1 CRUDE OIL/IBP-70 F

NID： 58262

Date: 10-JAN-1897

Analyzed: 2/4/97 6:43 PM

Reported: 02-05-1997 02:19:24 Normalized to $100.00 \%$

Comments :

Totals by Group Type \& Carbon Number (in Weight Percent)

Paraffins:

I-paraffins :

Aromatics: Naphthenes:

Olefins: Total:

$\begin{array}{lr}\text { C1: } & 0.000 \\ \text { C2: } & 0.033 \\ \text { C3: } & 8.803 \\ \text { C4: } & 49.185 \\ \text { C5: } & 10.924 \\ \text { C6: } & 0.256 \\ \text { C7: } & 0.000 \\ \text { C8: } & 0.000 \\ \text { C9: } & 0.000 \\ \text { C10: } & 0.000 \\ \text { C11: } & 0.000 \\ \text { C12: } & 0.000 \\ \text { C13: } & 0.000 \\ \text { C14: } & 0.000 \\ \text { Total: } & 69.201\end{array}$

0.000
0.000
0.000
9.586
10.792
0.416
0.026
0.000
0.000
0.000
0.000
0.000
0.000
0.000
20.820

0.000

0.000

0.000

0.000

0.000

0.000

0.000

0.000

0.000

0.034

0.740

0.000

0.646

0.000

0.008

0.000

0.000

0.000

0.000

0.000

0.000

0.000

0.000

0.000

0.000

0.000

0.000

0.000

0.034

1.394

0.000

0.000

0.000

0.033

0.210

9.013

6.105

64.876

2. 110

24.567

0.110

1.461

0.045

0.000

0.000

0.000

0.000

0.000

0.000

0.000

0.000

0.000

0.000

0.000

0.000

0.000

0.000

Oxygenates: $\quad 0.000 \quad$ Total CI4t: 0.000

Total Unknowns: 0.005

Grand Total: 100.000

Molecular Weight and Relative Density Data

$\begin{array}{rrr}\text { Group: } & \text { Ave. Mw.: } & \text { Ave. Rel. Density: } \\ \text { C1: } & 0.000 & 0.000 \\ \text { C2: } & 30.070 & 0.340 \\ \text { C3: } & 44.048 & 0.501 \\ \text { C4: } & 57.928 & 0.578 \\ \text { C5: } & 71.868 & 0.628 \\ \text { C6: } & 84.902 & 0.710 \\ \text { C7: } & 99.323 & 0.703 \\ \text { C8: } & 0.000 & 0.000 \\ \text { C9: } & 0.000 & 0.000 \\ \text { C10: } & 0.000 & 0.000 \\ \text { C11: } & 0.000 & 0.000 \\ \text { C12: } & 0.000 & 0.000 \\ \text { C13: } & 0.000 & 0.000 \\ \text { C14: } & 0.000 & 0.000 \\ \text { Sample: } & 59.337 & 0.582\end{array}$

File: $233-02 . D H A$ 
CALEB BRETT HOUSTON

TID: 97-000432-0-HOUS-002-00

CID: CONSOLINC

SID: HTI POC 1 CRUDE OIL/IBP-70 F

NID: 58262 Date: 10-JAN-1997

Analyzed: 2/4/97 6:43 PM

Reported: 02-05-1997 02:19:24

Normalized to $100.00 \%$

Comments :

Composite Report

Totals by Group Type \& Carbon Number

(in Volume Percent)

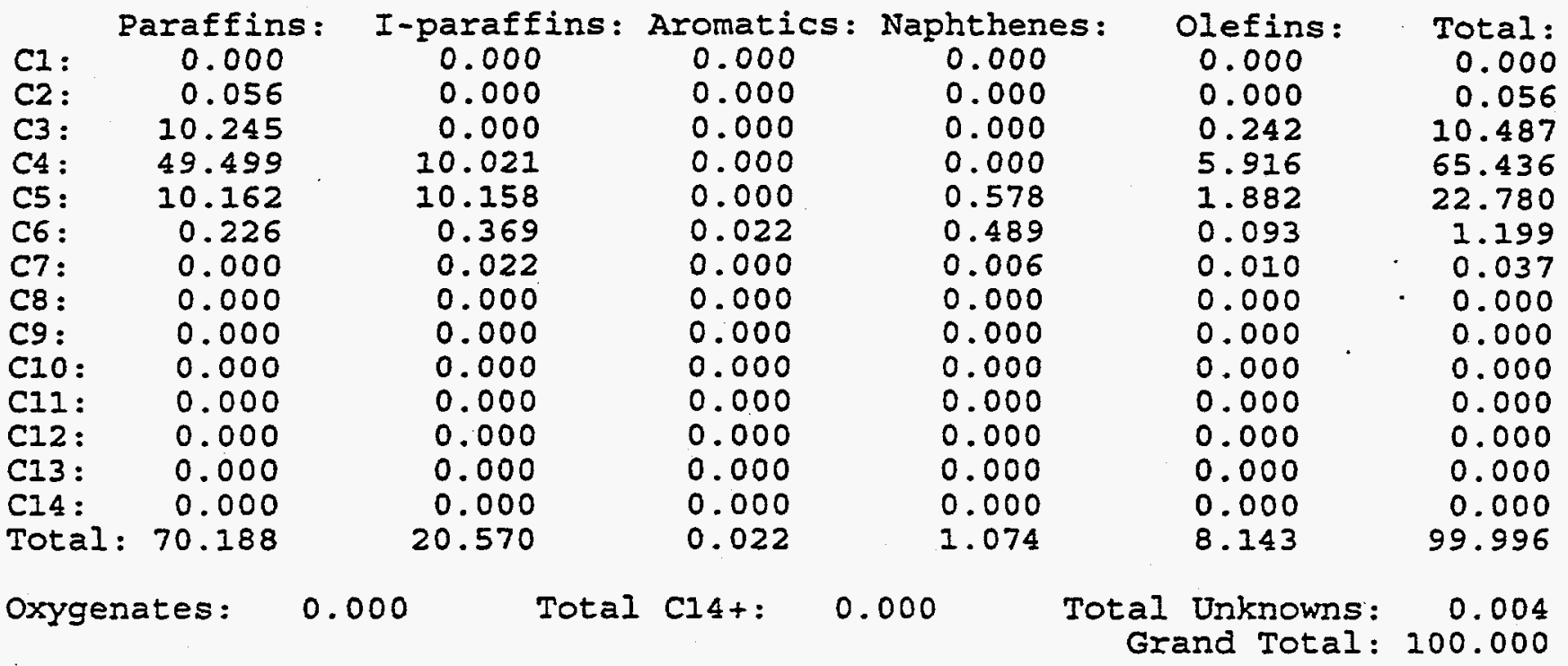

(in Mole Percent)

\begin{tabular}{|c|c|c|c|c|c|c|}
\hline $\begin{array}{l}\text { C1: } \\
\text { C2: } \\
\text { C3: } \\
\text { C4: } \\
\text { C5: } \\
\text { C6: } \\
\text { C7: } \\
\text { C8: } \\
\text { C9: } \\
\text { C10: } \\
\text { C11: } \\
\text { C12: } \\
\text { C13: } \\
\text { C14: } \\
\text { Total: }\end{array}$ & $\begin{array}{c}\text { Paraffins: } \\
0.000 \\
0.065 \\
11.846 \\
50.214 \\
8.984 \\
0.176 \\
0.000 \\
0.000 \\
0.000 \\
0.000 \\
0.000 \\
0.000 \\
0.000 \\
0.000 \\
: 71.285\end{array}$ & $\begin{array}{c}\text { I-paraffins: } \\
0.000 \\
0.000 \\
0.000 \\
9.787 \\
8.876 \\
0.286 \\
0.015 \\
0.000 \\
0.000 \\
0.000 \\
0.000 \\
0.000 \\
0.000 \\
0.000 \\
18.964\end{array}$ & $\begin{array}{l}\text { Aromatics: } \\
0.000 \\
0.000 \\
0.000 \\
0.000 \\
0.000 \\
0.026 \\
0.000 \\
0.000 \\
0.000 \\
0.000 \\
0.000 \\
0.000 \\
0.000 \\
0.000 \\
0.026\end{array}$ & $\begin{array}{c}\text { Naphthenes: } \\
0.000 \\
0.000 \\
0.000 \\
0.000 \\
0.626 \\
0.456 \\
0.005 \\
0.000 \\
0.000 \\
0.000 \\
0.000 \\
0.000 \\
0.000 \\
0.000 \\
1.087\end{array}$ & $\begin{array}{l}\text { Olefins: } \\
0.000 \\
0.000 \\
0.296 \\
6.457 \\
1.797 \\
0.078 \\
0.007 \\
0.000 \\
0.000 \\
0.000 \\
0.000 \\
0.000 \\
0.000 \\
0.000 \\
8.635\end{array}$ & $\begin{array}{r}\text { Total: } \\
0.000 \\
0.065 \\
12.142 \\
66.458 \\
20.284 \\
1.021 \\
0.027 \\
0.000 \\
0.000 \\
0.000 \\
0.000 \\
0.000 \\
0.000 \\
0.000 \\
99.997\end{array}$ \\
\hline
\end{tabular}

Oxygenates: 0.000 Total C14t: 0.000 Total Unknowns: 0.003 Grand Total: 100.000

File: 233-02.DHA 
CALEB BRETT HOUSTON

TID: 97-000432-0-HOUS-002-00

CID: CONSOLINC

SID: HTI POC 1 CRUDE OIL/IBP-70 F

NID: 58262

Date: 10-JAN-1997

Analyzed: 2/4/97 6:43 PM

Reported: 02-05-1997 02:19:24 Normalized to $100.00 \%$

Comments :

$(0.5 \%)$
$5.0 \%$
$10.0 \%$
$15.0 \%$
$20.0 \%$
$25.0 \%$
$30.0 \%$
$35.0 \%$
$40.0 \%$
$45.0 \%$
$50.0 \%$
$55.0 \%$
$60.0 \%$
$65.0 \%$
$70.0 \%$
$75.0 \%$
$80.0 \%$
$85.0 \%$
$90.0 \%$
$95.0 \%$
$(99.5 \%)$

Boiling Point Distribution Data

Wt. Percent Off: deg.C.: deg.F.:

$\begin{array}{rr}-42.04 & -43.67 \\ -42.04 & -43.67 \\ -11.72 & 10.90 \\ -11.72 & 10.90 \\ -6.25 & 20.75 \\ -0.50 & 31.10 \\ -0.50 & 31.10 \\ -0.50 & 31.10 \\ -0.50 & 31.10 \\ -0.50 & 31.10 \\ -0.50 & 31.10 \\ -0.50 & 31.10 \\ -0.50 & 31.10 \\ -0.50 & 31.10 \\ -0.50 & 31.10 \\ 27.84 & 82.11 \\ 27.84 & 82.11 \\ 27.84 & 82.11 \\ 36.06 & 96.91 \\ 36.06 & 96.91 \\ 80.72 & 177.30\end{array}$

Vol. Percent off: deg.C.: $\operatorname{deg.F.:~}$

$$
\begin{array}{r}
-42.04 \\
-42.04 \\
-42.04 \\
-11.72 \\
-11.72 \\
-0.50 \\
-0.50 \\
-0.50 \\
-0.50 \\
-0.50 \\
-0.50 \\
-0.50 \\
-0.50 \\
-0.50 \\
-0.50 \\
0.88 \\
27.84 \\
27.84 \\
36.06 \\
36.06
\end{array}
$$$$
71.80
$$$$
-43.67
$$$$
-43.67
$$$$
-43.67
$$$$
10.90
$$$$
10.90
$$$$
31.10
$$$$
31.10
$$$$
31.10
$$$$
31.10
$$$$
31.10^{\circ}
$$$$
31.10
$$$$
31.10
$$$$
31.10
$$$$
31.10
$$$$
31.10
$$$$
33.58
$$

82.11

82.11

96.91

96.91

161.24

Research Octane Number $=106.66$ (Calculated from Individual Component Values)

$\begin{array}{lc}\text { Contribution to Total by: } \\ \text { Paraffins: } & 73.18 \\ \text { Iso-paraffins: } & 22.64 \\ \text { Aromatics: } & 0.03 \\ \text { Naphthenes: } & 1.22 \\ \text { Olefins: } & 9.58 \\ \text { Oxygenates: } & 0.00\end{array}$

File: 233-02.DHA 
CALEB BRETT HOUSTON

TID: 97-000432-0-HOUS-002-00

CID: CONSOLINC

SID: HTI POC 1 CRUDE OIL/IBP-7O F

Analyzed: 2/4/97 6:43 PM

NID: 58262 Date: $10-J A N-$ I:997

Reported: 02-05-1997 02:19:24

Normalized to $100.00 \%$

Comments :

Components Listed in Chromatographic order

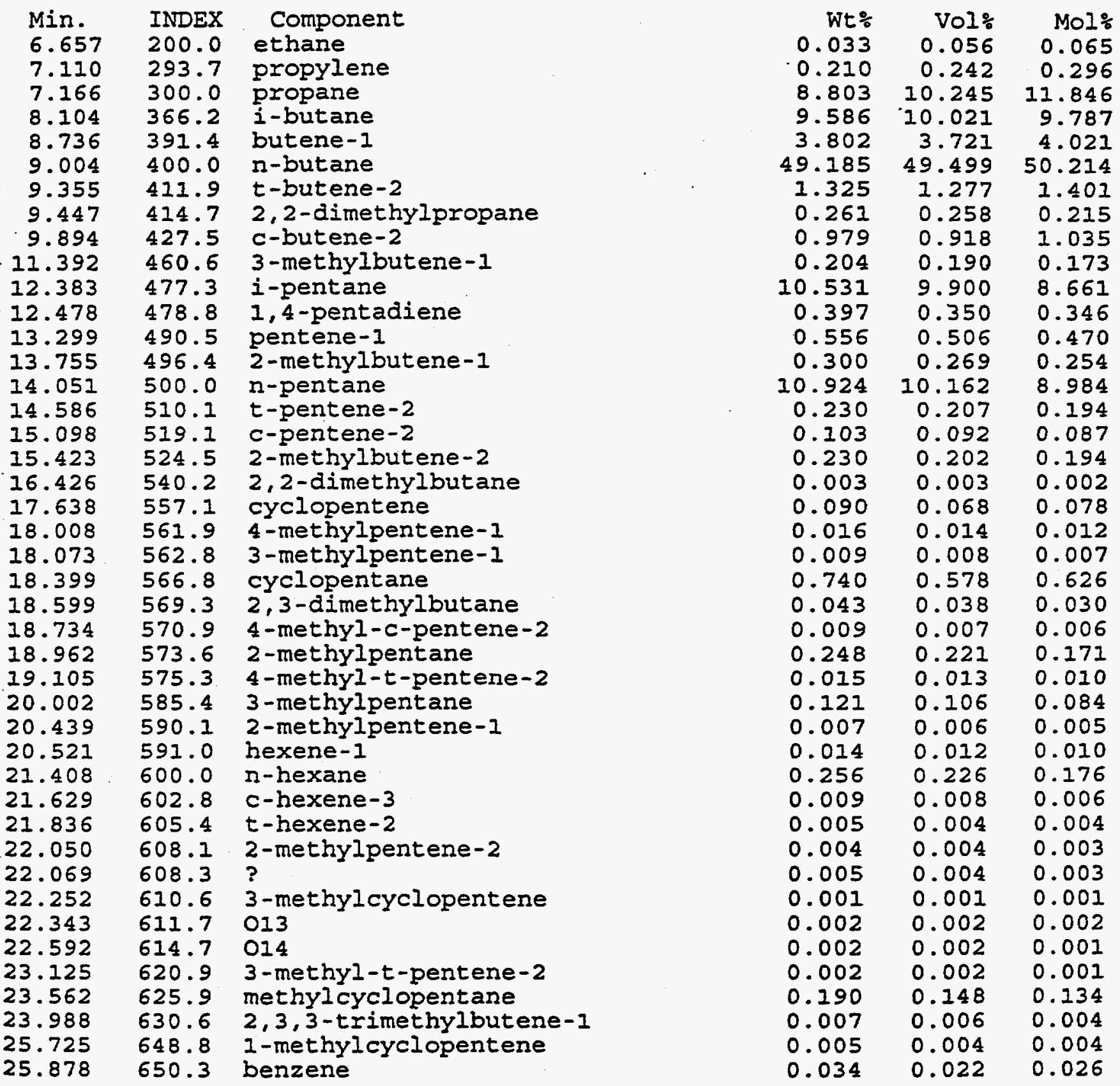

File: 233-02.DHA Sample: 97-233-2

p. 1 
Components Iisted in Chromatographic order

$\begin{array}{ccl}\text { Min. } & \text { INDEX } & \text { Component } \\ 26.663 & 657.9 & \text { cyclohexane } \\ 27.705 & 667.6 & 2,3-\text { dimethylpentane } \\ 27.850 & 668.9 & 5 \text {-methyl-t-hexene-2 } \\ 28.401 & 673.8 & \text { cyclohexene } \\ 28.650 & 675.9 & 3-\text { methylhexane } \\ 29.334 & 681.8 & \text { lc,3-dimethylcyclopentane }\end{array}$

$\begin{array}{lll}\text { Wt: } & \text { Vol\% } & \text { Mol\% } \\ 0.456 & 0.341 & 0.321 \\ 0.011 & 0.010 & 0.007 \\ 0.005 & 0.004 & 0.003 \\ 0.008 & 0.006 & 0.006 \\ 0.014 & 0.012 & 0.008 \\ 0.008 & 0.006 & 0.005\end{array}$

File: 233-02.DHA ... Sample: 97-233-2

p. 2 


\section{APPENDIX 3 \\ REPORT ON PREPARATION OF \\ ALKYL ARYL ETHERS FROM COAL DRIVED PHENOLS}

By: G. W. Heunisch 


\title{
PREPARATION OF ALKYL ARYL ETHERS FROM COAL-DERIVED PHENOLS
}

\author{
G. W. Heunisch \\ CONSOL Inc. \\ Research \& Development \\ 4000 Brownsville Road \\ Library, PA 5129
}

\section{INTRODUCTION AND SUMMARY}

CONSOL R\&D evaluated reactions to synthesize alkyl phenyl ethers from coal liquefaction phenols. The program included a literature review and laboratory chemical syntheses.

Phenols are produced during the direct liquefaction of coal and must be removed prior to producing transportation fuels. The crude liquefaction product is commonly hydrotreated to remove the phenols and other unsaturated components. An alternative method is to extract the phenols from the crude coal liquefaction product and use them in other commercial processes. Hydrogen consumption for the hydrotreatment would be reduced, and the phenolic material could be converted to alkylphenyl ethers. Alkylphenyl ethers may be useful as fuel extenders. If the fuel extenders can be produced using grain alcohol, the products may be entitled to special tax considerations.

An extensive literature search identified the Williamson Synthesis and its modifications as the preferred methods to produce mixed ethers from phenols. A variation of the Williamson Synthesis ${ }^{(1,2,3)}$ was used to produce phenetole and the ethyl derivatives of the phenolics from a caustic extract of a crude direct coal liquefaction product. The methyl derivatives of similar coal derived phenolic material were synthesized and characterized earlier at Gulf Research. ${ }^{(4)}$ Other approaches involving acid catalysts failed to produce ethers.

\section{RECOMMENDATION}

The ethyl ether derivatives of coal liquefaction phenols should be synthesized and characterized as a diesel fuel extender. CONSOL R\&D will develop a plan to extract and synthesize the ethers and coordinate the fuel characterization upon DOE's approval.

\section{DISCUSSION}

\section{Literature Search}

A literature search was conducted to identify commercial processes which produce alkylphenyl ethers. The Williamson Synthesis and its modifications are the methods commonly used to produce mixed ethers from phenols, although other innovative laboratory methods were identified. The DOE-FETC library, the NIOSH (formerly the Bureau of Mines) library, and the extensive electronic data base of Dialog, Inc. of Knight-Ridder, Inc. were searched. A former Gulf Research employee who prepared and tested methylphenyl ethers as gasoline additives was consulted. 


\section{Williamson Synthesis}

Phenetole. Phenetole, ethylphenyl ether, was prepared to verify the chemical procedure and the analytical techniques. A variation of the Williamson Synthesis was used to prepare the ether. Phenol was reacted with diethylsulfate in the presence of sodium hydroxide under reflux conditions. On cooling, an organic liquid phase separated from the aqueous solution. An ${ }^{1} \mathrm{H}$ NMR analysis (Figure 1a) of the isolated organic phase indicated a mixture of phenetole and residual diethylsulfate. An analysis of the organic phase showed $11.9 \%$ sulfur, which indicates $57 \%$ diethylsulfate. The diethylsulfate was removed by hydrolysis of the mixture in warm water. Figure $1 \mathrm{~b}$ shows an ${ }^{1} \mathrm{H}$-NMR spectrum of the purified phenetole. The infrared spectrum shows the characteristic absorption for the ether linkage at $1250 \mathrm{~cm}^{-1}$ and $1025 \mathrm{~cm}^{-1}$, ${ }^{(5)}$ and the organic product was identified by GC/MS as phenetole with trace impurities. The yield was $40-50 \%$ of the theoretical yield. The yield can be improved by optimizing the experimental conditions. The phenolic $-\mathrm{OH}$ concentration in the product was determined by an FTIR procedure to be 0.001 $\mathrm{meq} / \mathrm{g}$, indicating the absence of phenol.

Ethylated Coal Liquid. The phenols extracted from the IBP $-380^{\circ} \mathrm{F}$ distillate fraction of the crude coal liquid from Black Thunder coal was treated with diethylsulfate under the same experimental conditions used to prepare phenetole. The source of the coal liquid is identified in the Experimental Section. The ${ }^{1} \mathrm{H}$-NMR spectrum of the organic mixture showed the diethylsulfate impurity, and an analysis gave $10.0 \%$ sulfur. The liquid was hydrolyzed to remove the diethylsulfate. The hydrolyzed product contained $0.2 \%$ sulfur.

A comparison of the ${ }^{1} \mathrm{H}$-NMR spectra of the original phenolic fraction and the ethylated product is presented in Figure 2. The spectra show the disappearance of the phenol group at $6.6 \mathrm{ppm}$, splitting of the side chain methyl groups, and an ethyl group bound to an electronegative component at 1.3 and $3.9 \mathrm{ppm}$; e.g., oxygen. The infrared spectrum shows characteristic ether linkage absorptions at $1250 \mathrm{~cm}^{-1}$ and $1025 \mathrm{~cm}^{-1}$. GC/MS identified phenetole, dimethylphenetole, and other methyl and/or ethyl substituted phenetoles. The concentration of phenolic $-\mathrm{OH}$ was $<0.2 \mathrm{meq} / \mathrm{g}$, indicating zero or trace residual phenol.

The phenols extracted from the $380^{\circ}-510^{\circ} \mathrm{F}$ fraction of the Black Thunder coal liquids were also treated with diethylsulfate under the same conditions. The ${ }^{1} \mathrm{H}-\mathrm{NMR}$ spectrum of the product is shown in Figure 3 and is analogous to that of the IBP- $380^{\circ} \mathrm{F}$ derived material. The GC/MS chromatogram shows alkyl substituted phenetoles corresponding to the starting phenols. Ethers with side chains containing up to four carbon atoms were identified. The characteristic ether absorption was observed at $1250 \mathrm{~cm}^{-1}$ and $1025 \mathrm{~cm}^{-1}$ in the infrared spectrum. The absorptions are not observed in the starting phenols.

\section{Acid Catalyzed Syntheses}

p-Toluenesulfonic Acid. Based on a conversation with G. Singerman, formerly of Gulf Research, methanol and ethanol were reacted with phenol under reflux in the presence of p-toluenesulfonic acid. The etherification reaction is expected to proceed as shown below: 


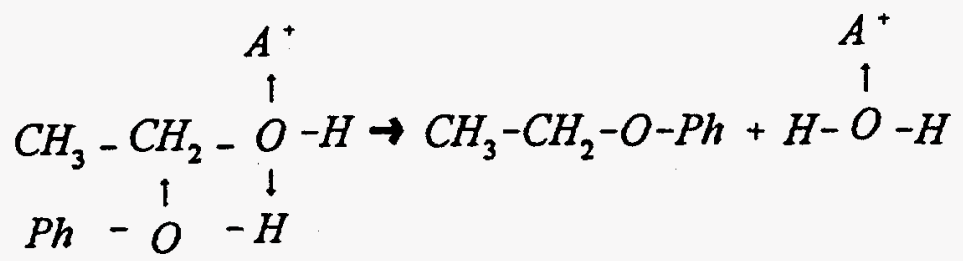

The comparison of ${ }^{1} \mathrm{H}$-NMR spectra shown in Figure 4 indicate that no reaction occurred in the acid solution. Figure 4a shows the ${ }^{1} \mathrm{H}$-NMR spectrum of the methanol/phenol/p-toluenesulfonic acid reaction product. Figure $4 \mathrm{~b}$ is a spectrum of $50 \%$ phenol in methanol solution. Figures $4 a$ and $4 \mathrm{c}$ are analogous spectra for phenol with ethanol. The spectra of the reaction products do not show the characteristic shifts attributed to anisole or phenetole and are indistinguishable from a solution of phenol in the appropriate alcohol. The peak at $6.7 \mathrm{ppm}$ in Figures $4 \mathrm{a}$ and $4 \mathrm{c}$ is attributed to the phenolic $-\mathrm{OH}$, which is protonated in the acid solution.

The same reagents were reacted in the presence of $15 \%$ sulfuric acid solution. Again, only the starting materials were observed by ${ }^{1} \mathrm{H}-\mathrm{NMR}$ in the reaction product.

Attempts to react alcohols and phenol were unsuccessful in the presence of hydrated boron trifluoride, a Lewis acid. The ${ }^{1} \mathrm{H}-\mathrm{NMR}$ spectra showed only the starting materials. GC/MS shows phenol as the major component. No ethers were observed.

\section{EXPERIMENTAL}

\section{Phenetole Preparation}

Phenetole was prepared by reacting reagent grade phenol and diethylsulfate in basic sodium hydroxide solution. The phenol $(10.05 \mathrm{~g}$ ) was placed in a $250-\mathrm{mL}$ round bottom flask fitted with a single standard taper ground glass joint. The phenol was dissolved in $50 \mathrm{~mL}$ of $10 \%$ sodium hydroxide solution with stirring. Diethylsulfate $(12.1 \mathrm{~g})$ was added slowly with continued stirring, and a condenser was connected to the ground glass joint. The reaction mixture was refluxed for three hours with stirring. The apparatus was confined to a chemical ventilation hood. The solution was cooled, and $5 \mathrm{~mL}$ of $25 \%$ sodium hydroxide solution were added to decompose excess diethylsulfate. The two-phase mixture was transferred to a 200-mL separatory funnel and the aqueous layer was separated and discarded. The organic layer was washed twice with 25 $\mathrm{mL}$ aliquots of $10 \% \mathrm{NaOH} / 10 \% \mathrm{NaCl}$ solution. The washed organic product was analyzed using ${ }^{1} \mathrm{H}$-NMR, GC/MS, and infrared spectroscopy. To decompose and remove the excess diethylsulfate, the mixture was warmed with an equal volume of deionized water for two hours. The diethylsulfate destruction was confirmed by ${ }^{1} \mathrm{H}-\mathrm{NMR}$ and GC/MS.

\section{Etherification of Coal Liquid Phenols}

The procedure described above was used to prepare and purify the O-ethylated derivatives of the phenols derived from coal liquefaction. The phenols extracted from the IBP- $380^{\circ} \mathrm{F}$ and the $380^{\circ} \mathrm{F}-510^{\circ} \mathrm{F}$ distillation fractions from Black Thunder coal liquids were reacted with diethylsulfate and the products were analyzed by ${ }^{1} \mathrm{H}-\mathrm{NMR}, \mathrm{GC} / \mathrm{MS}$, and infrared spectroscopy. The IBP-380 ${ }^{\circ} \mathrm{F}$ distillate fraction, after reaction with diethylsulfate, yielded $7.3 \mathrm{~g}$ of ethylated product. The $380^{\circ} \mathrm{F}-510^{\circ} \mathrm{F}$ fraction yielded $8.6 \mathrm{~g}$ of recovered product. 


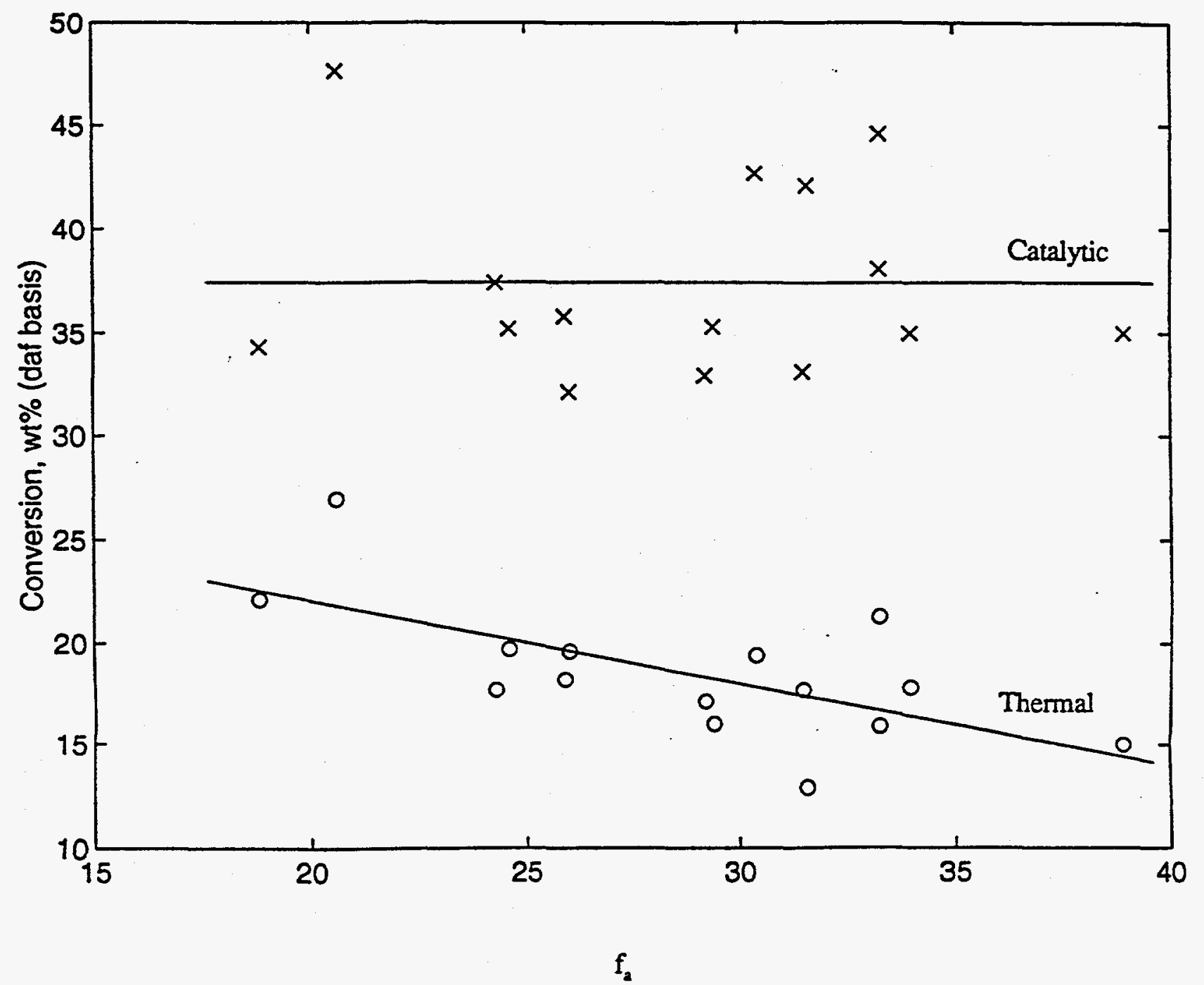

Figure 3 Thermal and catalyzed hydroprocessing conversions vs $f_{a}$ of the resids 


\section{Reaction of Phenol and Alcohols in Acid Media}

p-Toluenesulfonic acid ( $99 \%$ ), sulfuric acid (reagent grade), and hydrated boron trifluoride $(96 \%$ $\mathrm{BF}_{3} \cdot 2 \mathrm{H}_{2} \mathrm{O}$ ) were evaluated as catalysts. The phenol was placed in a $250-\mathrm{mL}$ round bottom flask and dissolved in the methyl or ethyl alcohol. After addition of the acid catalyst (4-8\%), the flask was fitted with a condenser. The reaction mixture was refluxed for 2.5 hours, cooled, and the total product mixture was analyzed for the appropriate ether.

The quantities of the reactants using p-toluenesulfonic acid are given in the table below:

\begin{tabular}{||l|c|c|c|}
\hline \multirow{2}{*}{ Reactant Alcohol } & \multicolumn{3}{|c|}{ Quantity } \\
\cline { 2 - 4 } & Phenol, g & Alcohol, mL & p-TSA,g \\
\hline Methyl & 22.03 & 14 & 2.06 \\
\hline Ethyl & 21.95 & 15 & 2.05 \\
\hline
\end{tabular}

The quantities of the reactants using $15 \%$ sulfuric acid are given in the following table:

\begin{tabular}{|l|c|c|c|}
\hline \multirow{2}{*}{ Reactant Alcohol } & \multicolumn{3}{|c|}{ Quantity } \\
\cline { 2 - 4 } & Phenol, g & Alcohol, $\mathrm{mL}$ & $15 \% \mathrm{H}_{2} \mathrm{SO}_{4}, \mathrm{~mL}$ \\
\hline Methyl & - & - & - \\
\hline Ethyl & 19.92 & 20 & 20 \\
\hline
\end{tabular}

The boron trifluoride catalyzed reaction mixtures produced precipitates, which were determined by infrared spectroscopy and ${ }^{19} \mathrm{~F}-\mathrm{NMR}$ to be silicon compounds etched from the flask. The quantities of the reactants are given in the following table:

\begin{tabular}{|l|c|c|c|}
\hline \multirow{2}{*}{ Reactant Alcohol } & \multicolumn{3}{|c|}{ Quantity } \\
\cline { 2 - 4 } & Phenol, $\mathrm{g}$ & Alcohol, $\mathrm{mL}$ & $\mathrm{BF}_{3} \cdot 2 \mathrm{H}_{2} \mathrm{O}, \mathrm{mL}$ \\
\hline Methyl & 22.34 & 11 & 5 \\
\hline Ethyl & 20.08 & 13 & 6 \\
\hline
\end{tabular}

None of the reactions in acid media produced a two-phase mixture. The crude reaction product was analyzed for the ether.

\section{EQUIPMENT AND CHEMICALS}

\section{Nuclear Magnetic Resonance ( $\left.{ }^{4} \mathrm{H}-\mathrm{r}^{9} \mathrm{~F}-\mathrm{NMR}\right)$}

The NMR spectra were obtained using a Varian Model EM360L NMR Spectrometer. Samples were dissolved in $\mathrm{CDCl}_{3}$ and referenced to tetramethylsilane. 


\section{Gas Chromatography/Mass Spectrometry (GC/MS)}

The GC/MS data were obtained using a Hewlett-Packard Model 5890, Series II, Gas Chromatograph equipped with a Hewlett-Packard Model 5970 Mass Selective Detector and a phenylmethylpolysiloxane column. Identifications were based on a search of the Wiley/NBS library of mass spectra.

\section{Infrared Spectroscopy}

The infrared spectra were obtained using a Nicolet Model 550 Magna-IR Spectrometer, Series II. A $0.2 \mathrm{~cm} \mathrm{NaCl}$ cell was used for the liquid samples. The phenolic $-\mathrm{OH}$ concentration in solution was determined using this instrument.

\section{Sulfur Determination}

The concentration of sulfur in the reaction mixtures was determined using a LECO Model SC-432 Sulfur Determinator.

\section{Coal-Derived Phenols}

The coal liquid phenols were obtained from a direct coal liquefaction product sample from HTI Run $\mathrm{CC}-15$. Black Thunder Coal was the feedstock. The crude product was distilled and the IBP-380 ${ }^{\circ} \mathrm{F}$ and $380^{\circ} \mathrm{F}-510^{\circ} \mathrm{F}$ distillate fractions were extracted with caustic to isolate the corresponding phenolic fraction by the National Institute for Petroleum and Energy Research (6).

\section{REFERENCES}

1. Encyclopedia of Chemical Technology, H. E. Mark, J. J. McKetta, Jr., D. F. Othmer, Editors, Vol. 5, 2nd Ed., Interscience Publishers, Inc., NY, 1965.

2. The Chemistry of the Ether Linkage, S. Patai, Editor, Interscience Publishers, NY, 1967.

3. Encyclopedia of Science and Technology, McGraw-Hill Book Co., NY, 1971.

4. Singerman, G. M., "Methyl Aryl Ethers from Coal Liquids as Gasoline Extenders and Octane Improvers", Gulf Research and Development Co., Report DOE/CE/50022-1, November 1980.

5. Spectrometric Identification of Organic Compounds, R. M. Silverstein and G. C. Bassler, John Wiley \& Sons, Inc., NY, 1963.

6. Sturm, G. P., Jr., Kim, J., Shay, J., "Coal Liquefaction Process Streams Characterization and Evaluation/Analysis of Coal-Derived Synthetic Crude from HRI CTSL Run CC-15 and HRI Run CMSL-2", Report DOE/PC89883-92, January 1994. 


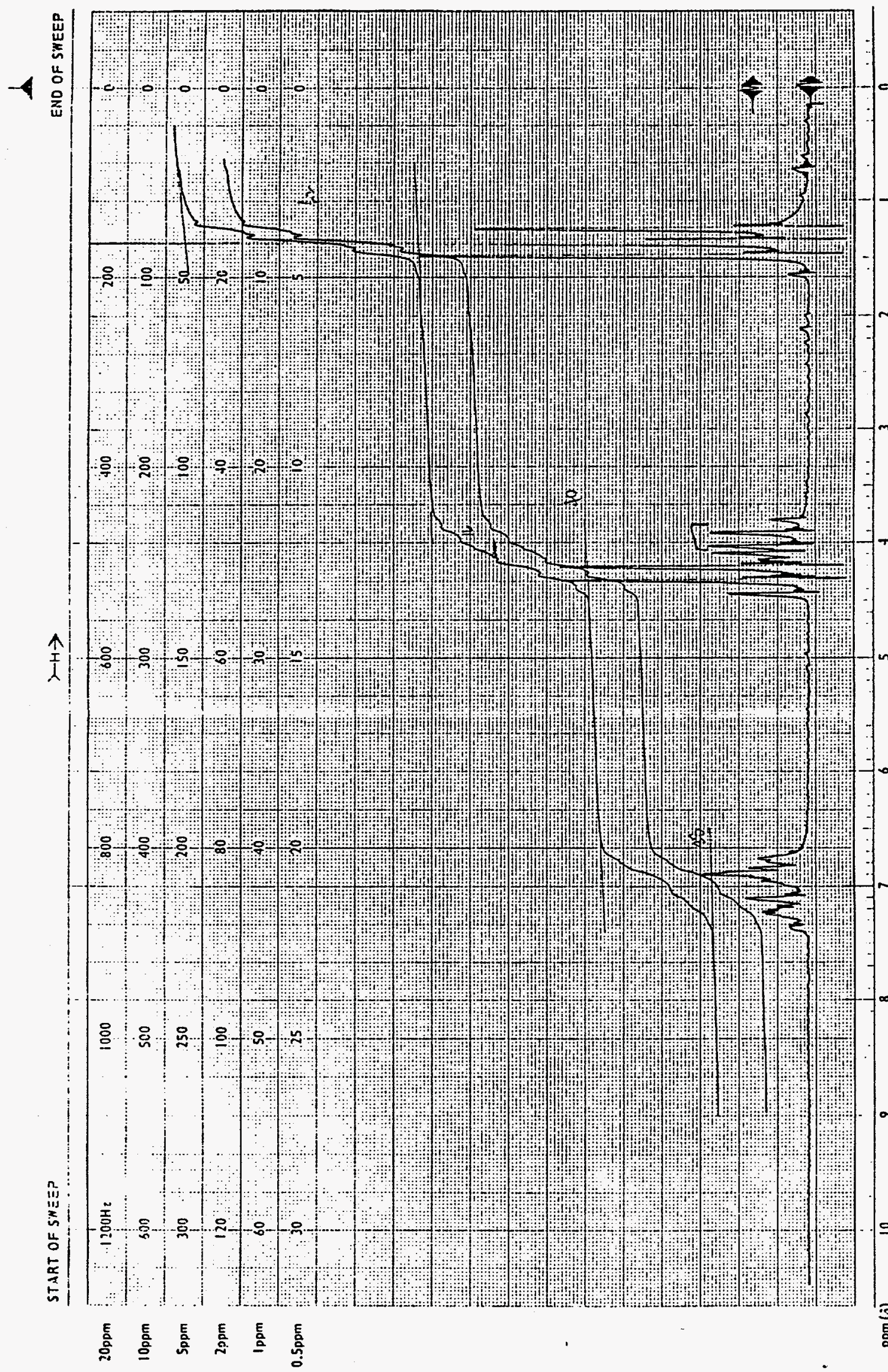




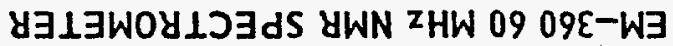

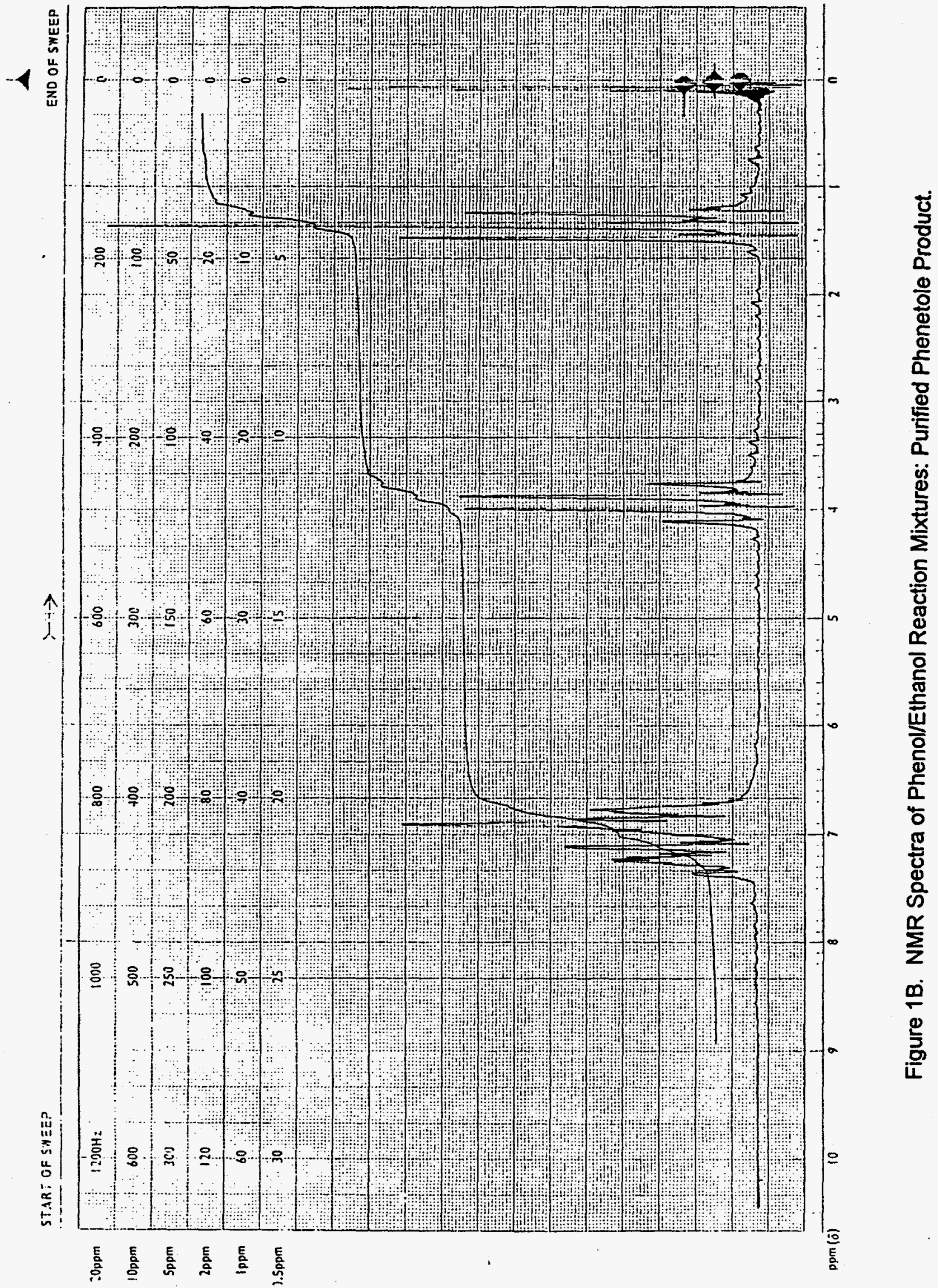




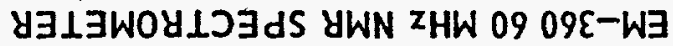
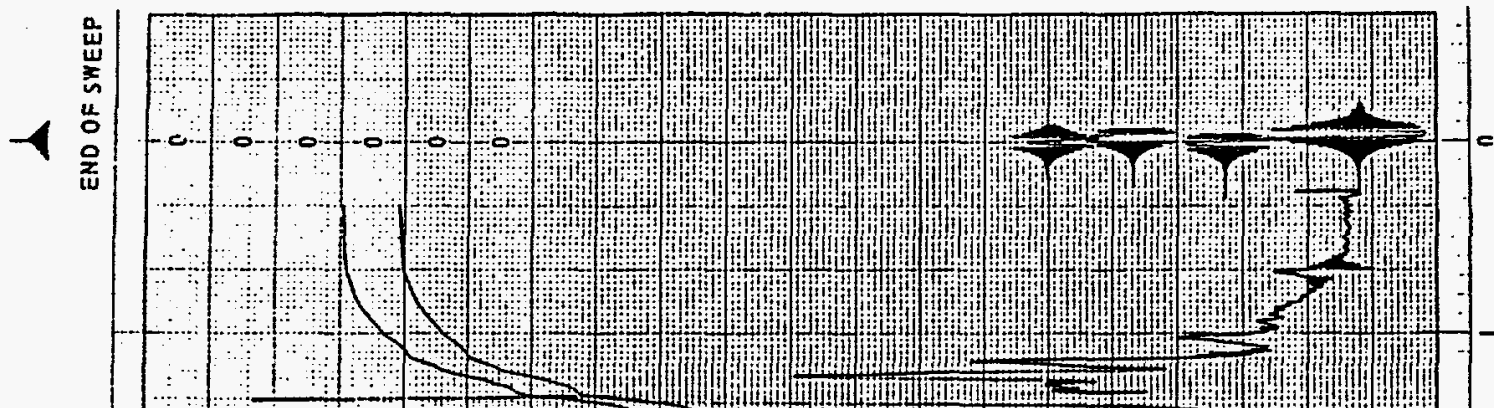

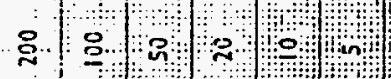
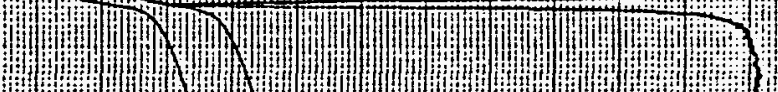

a (u)

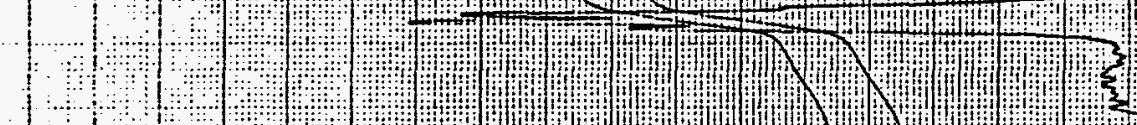

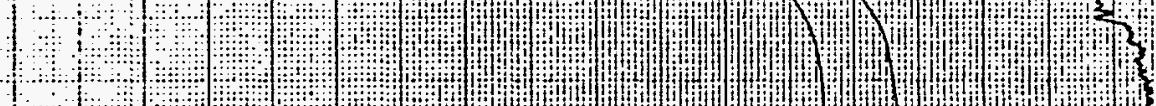

(1)

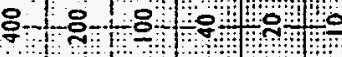

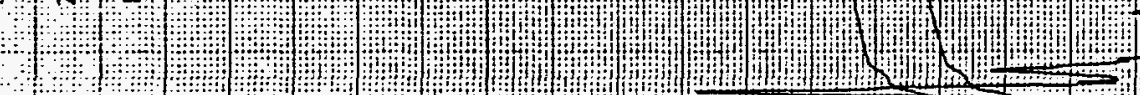

^

entolutolo

(1) (1)
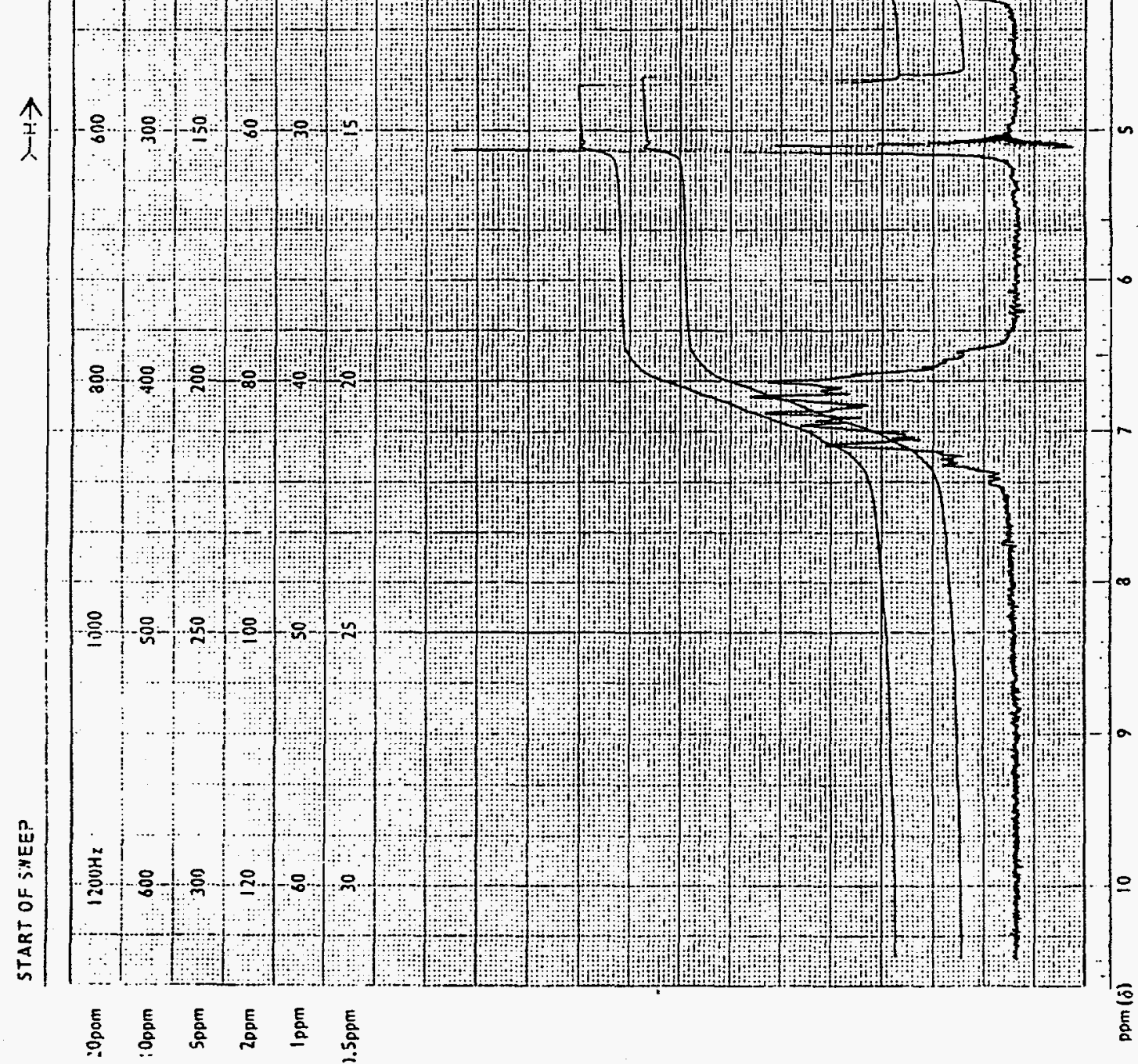

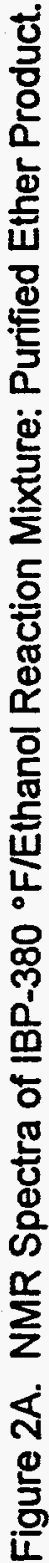




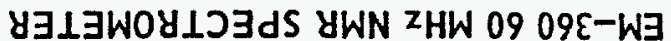

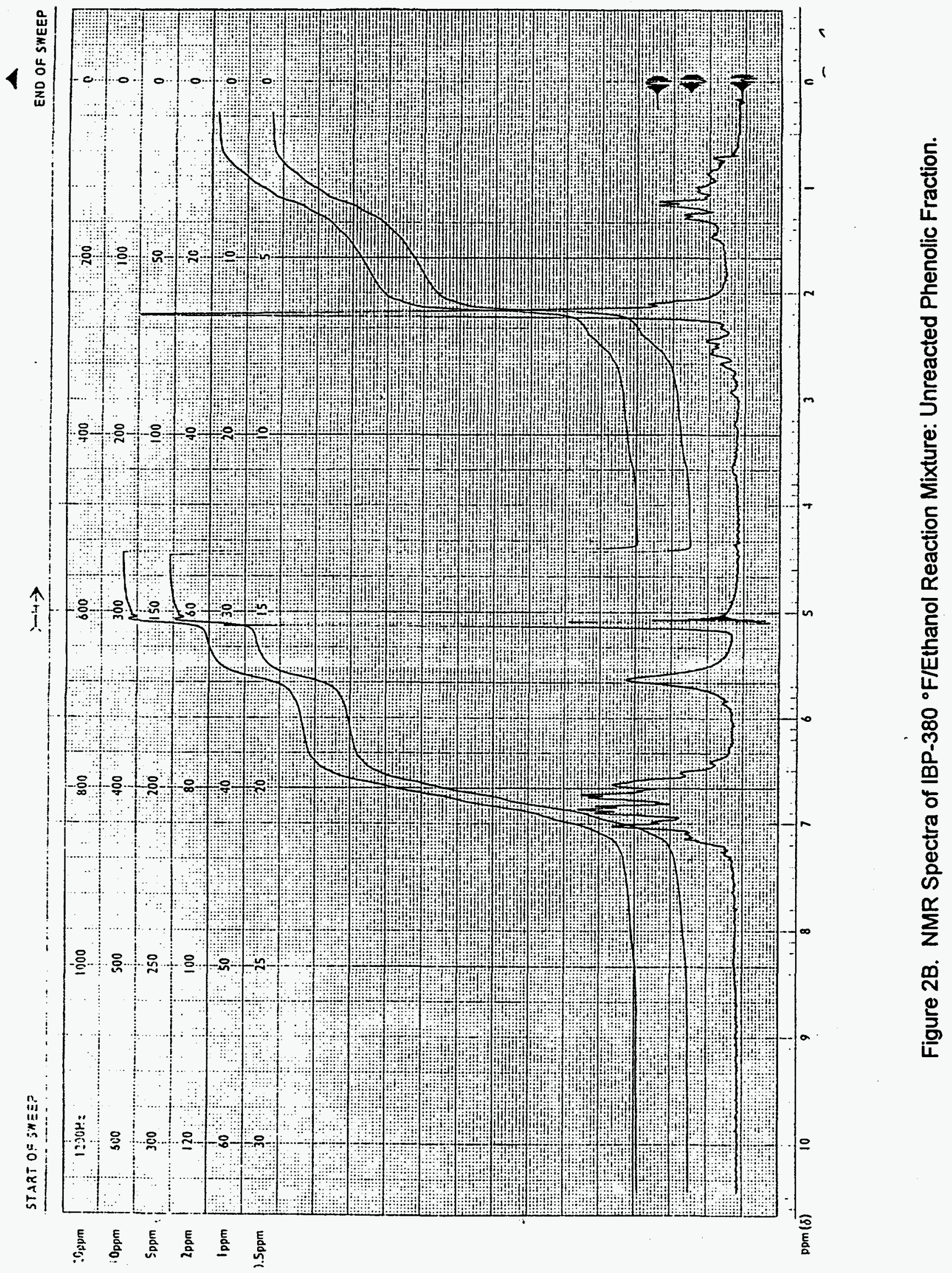


У $\lrcorner \perp \exists W O Y \perp \supset \exists d S$ UWN ZHW 09 09E-W

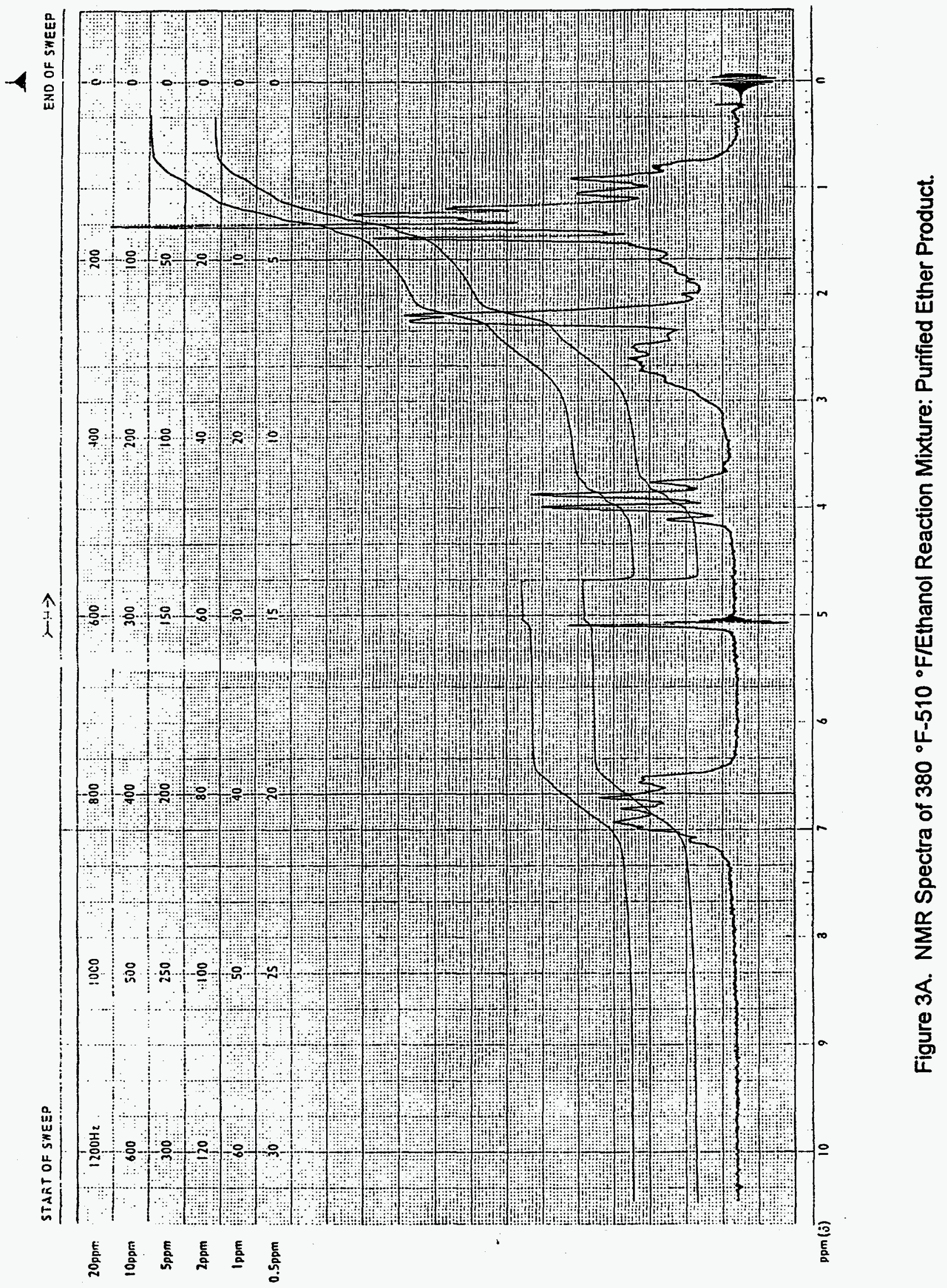




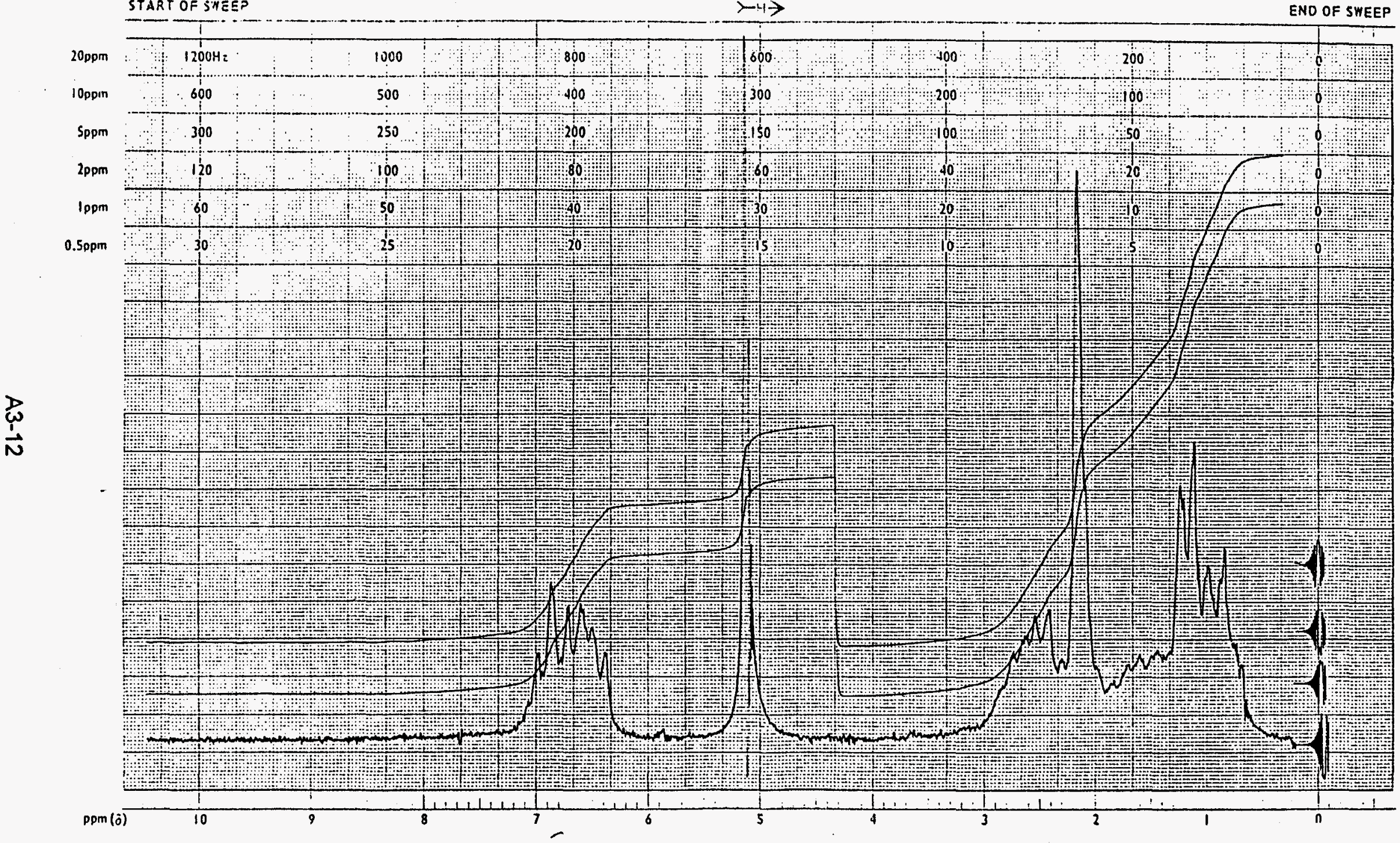

Figure 3B. NMR Spectra of $380^{\circ} \mathrm{F}-510^{\circ} \mathrm{F} /$ Ethanol Reaction Mixture: Unreacted Phenolic Fraction. 
20ppm

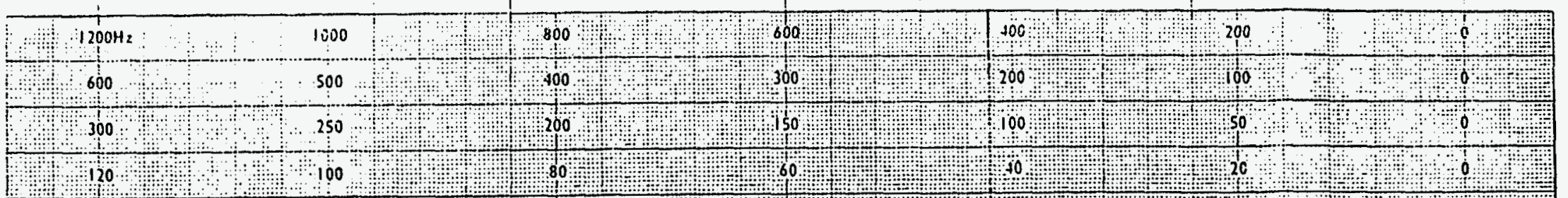

$2 \mathrm{ppm}$

Ippm

$0.5 \mathrm{pgm}$

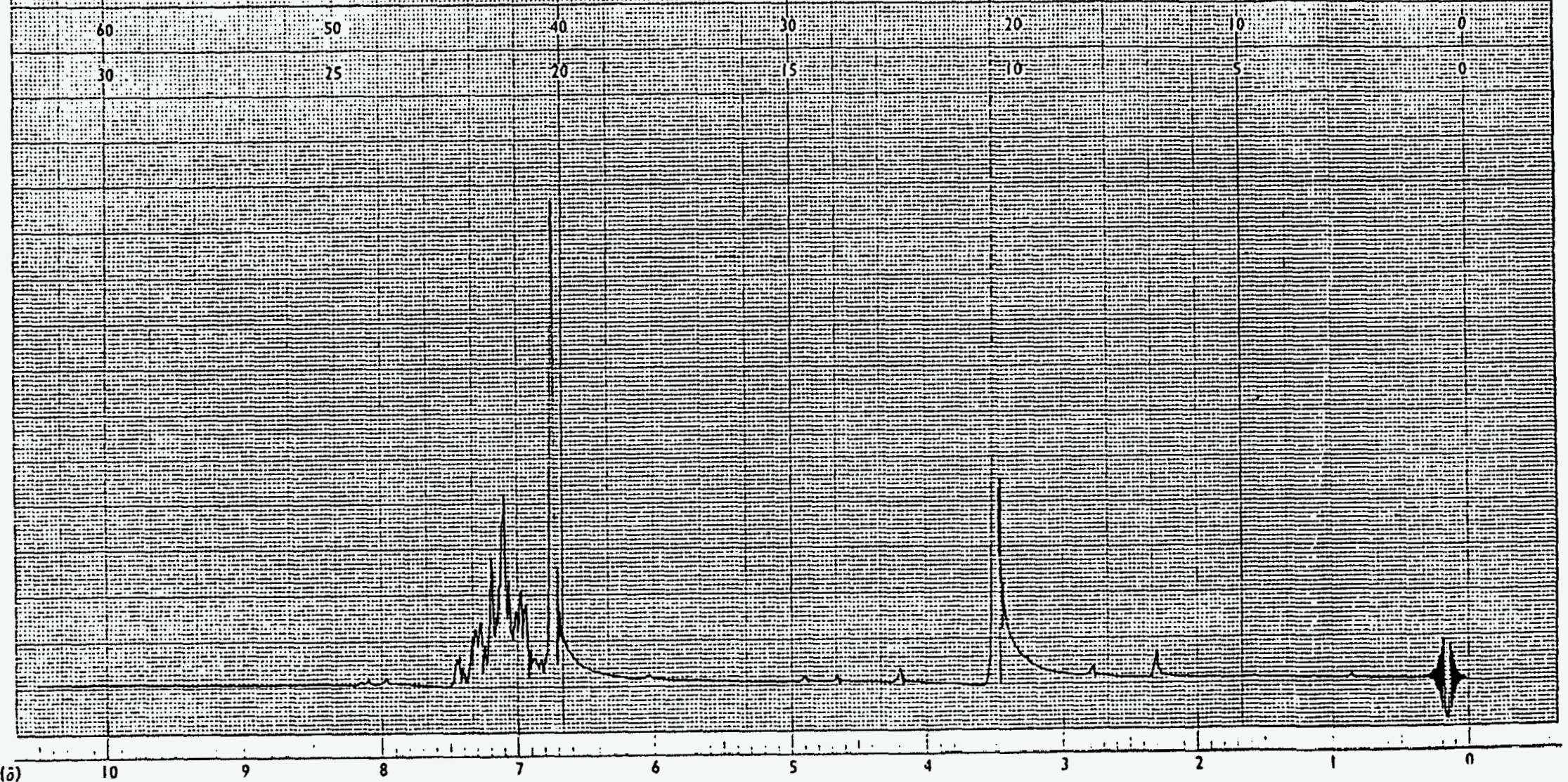

Figure 4A. NMR Spectra of Phenol/Alcohol Mixtures: Phenol/Methanol/p-Toluenesulfonic Acid Reaction Product. 


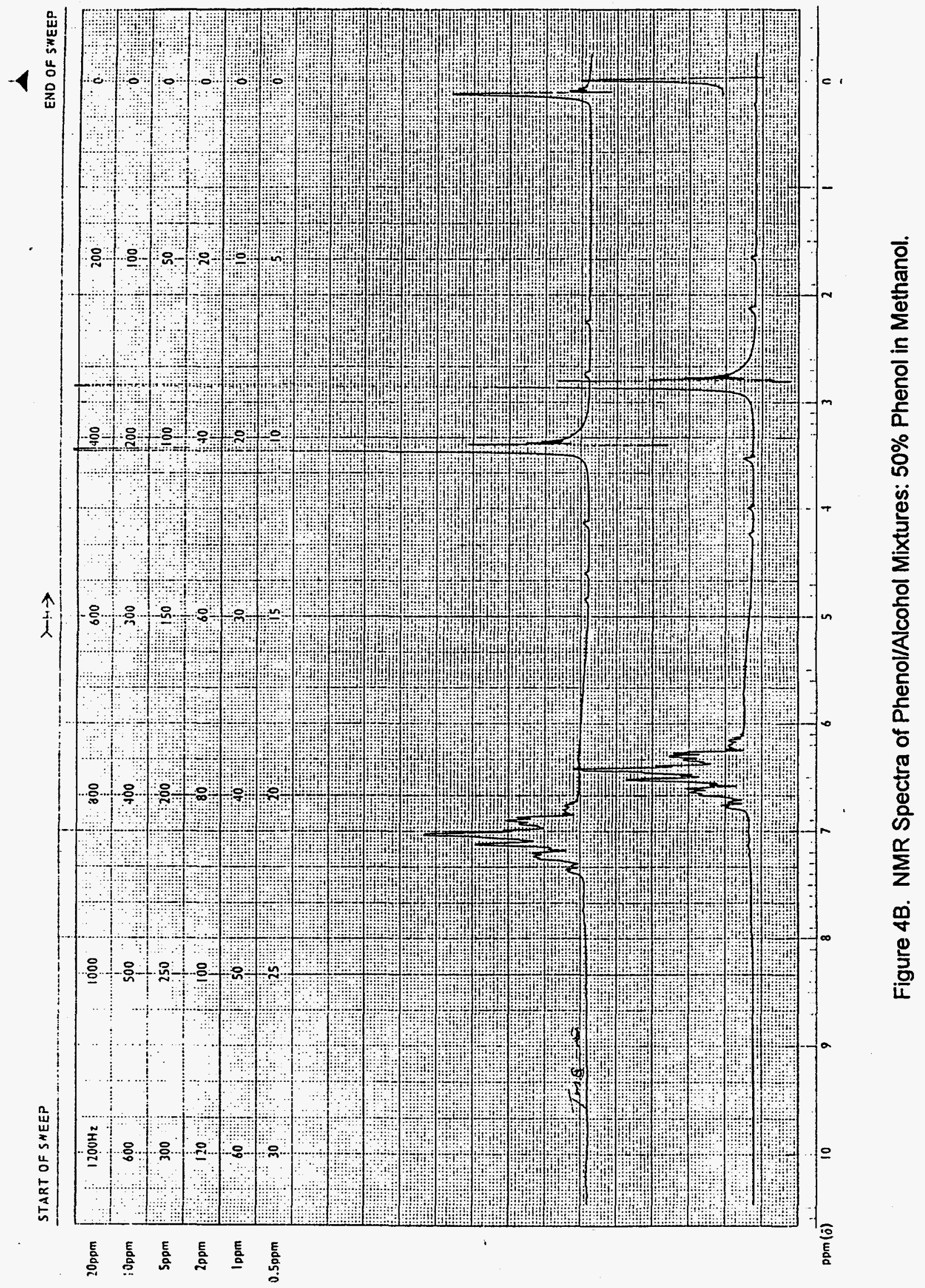




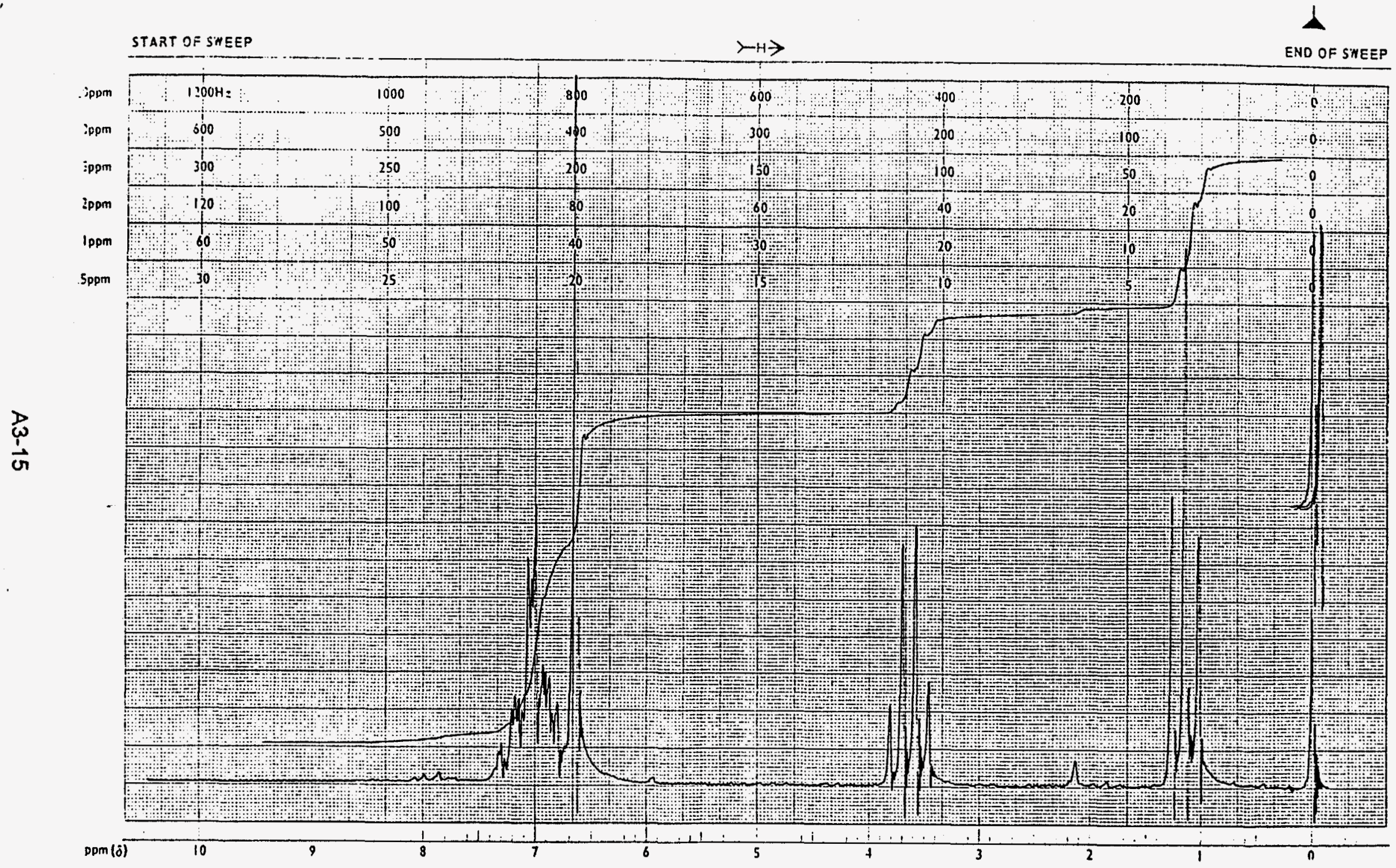

Figure 4C. NMR Spectra of Phenol/Alcohol Mixtures: Phenol/Ethanol/p-Toluenesulfonic Acid Reaction Product. 


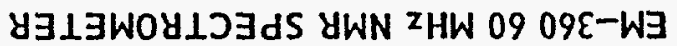
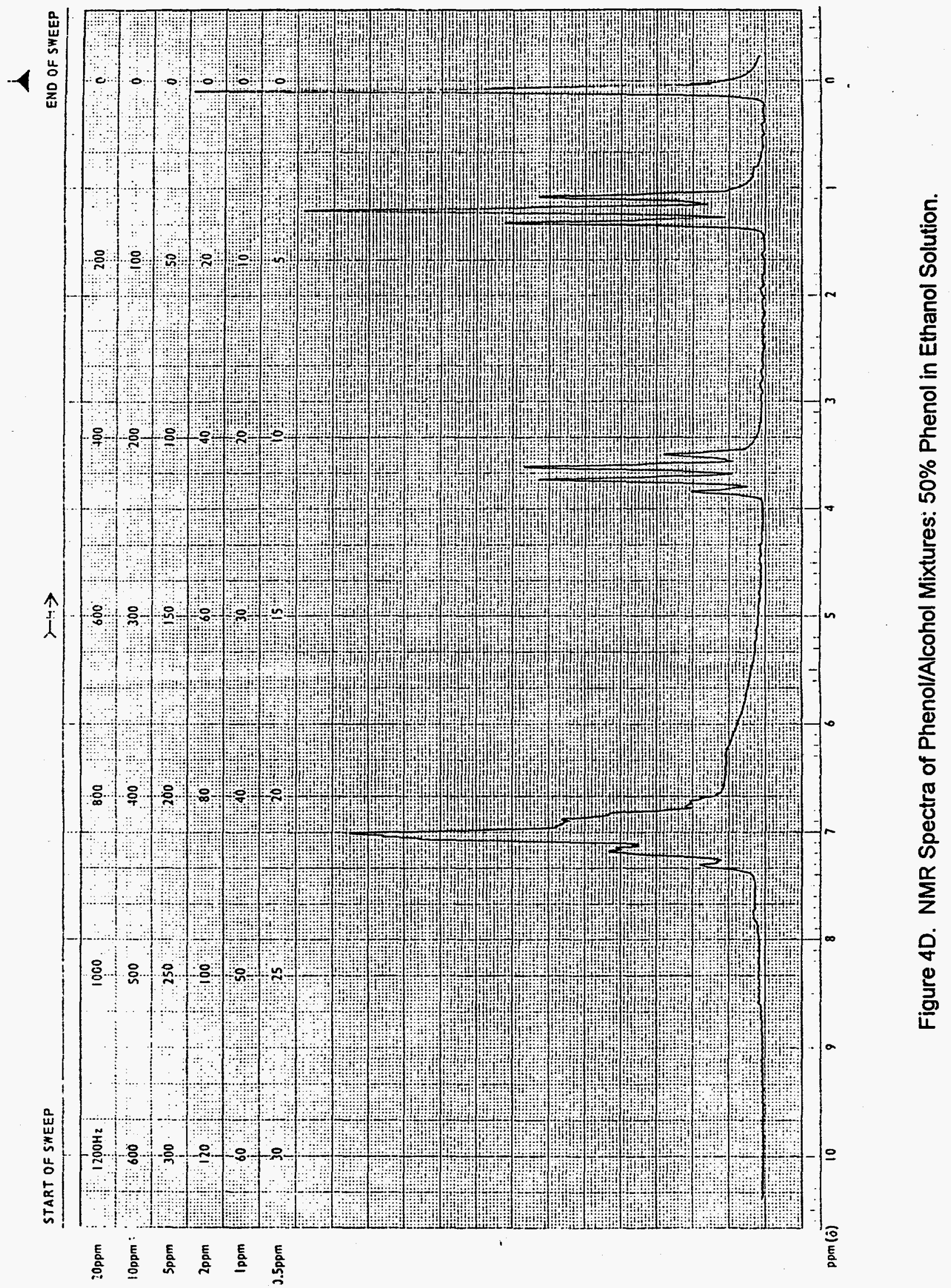


\section{APPENDIX 4}

\section{SAMPLES SUPPLIED TO OTHER DOE PROJECTS}

A4-1 
December 12, 1996

CONSOL Inc.

Research \& Development 4000 Brownswille Road

Library, PA 15129.9566

412.854 .6600

Fax: $+12-854-50 \hat{13}$

$412-854-6683$

Dr. John Zondlo

Department of Chemical Engineering

West Virginia University

P. O. Box 6101

Morgantown, WV 26506-6101

Dear Dr. Zondlo:

Dr. Michael A. Nowak of DOE/FETC asked if I could provide you with a coal liquefaction material for possible use in making anodes. I am shipping you a $4 \mathrm{oz} \mathrm{jar} \mathrm{of} \mathrm{a} \mathrm{material} \mathrm{labeled}$ "Kerr-McGee Light Phase" for your inspection. I have a $55 \mathrm{gal}$ drum of this material and one drum of a similar material. What follows is my best attempt to reconstruct the source of this material; be aware that I am uncertain of many details. I believe the material was produced at the Wilsonville, AL, Solvent Refined Coal (SRC) pilot plant. The Kerr-McGee critical Solvent Deasher (CSD, later called the ROSE-SR unit) was a device that deashed the raw SRC product of the liquefaction section of the plant. The CSD unit produced an "ash concentrate" stream and "light SRC" and "heavy SRC" streams. The light and heavy SRC streams were sometimes combined and sometimes left separate. I believe this material is the light SRC stream produced in about 1979 from a Wilsonville run made with an Illinois Basin bituminous coal. Our laboratory recently obtained the following elemental analysis of the sample:

$\begin{array}{ll} & \text { wt \% } \\ \mathrm{C} & 85.71 \\ \mathrm{H} & 6.73 \\ \mathrm{~N} & 1.80 \\ \mathrm{~S} & 0.68\end{array}$

We also obtained a ${ }^{1} \mathrm{H}$ - nuclear magnetic resonance spectrum of the sample; $38.5 \%$ of the hydrogen is aromatic.

An MSDS is enclosed with the sample. Please call me to discuss the sample origins or to request a drum of the material.

Sincerely,

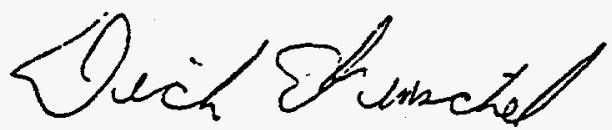

R. A. Winschel

Research Group Leader

ns

CC: M. A. Nowak

R. M. Statnick 
CONSOL Inc.

Research \& Development 4000 Brovinsilile Road

Library. PA 15129.9566

$412-854-6600$

FAI $412-854-6613$

412-854-6568

Dr. John Zondlo

Department of Chemical Engineering

West Virginia University

P. O. Box 6101

Morgantown, WW 26506-6101

Dear Dr. Zondio:

Dr. Michael A. Nowak of DOE-FETC informed me that a sample sent to you in December 1996 by Dick Winschel of CONSOL R\&D was found by you to be inappropriate for the production of carbon anodes. Mike requested that I supply you with another sample that would be representative of a "typical" two-stage direct coal liquefaction process stream.

The sample I have chosen to send is a composite of individual samples taken over extended periods of Run 259 made in 1990 at the Wilsonville integrated two-stage coal liquefaction pilot plant. Wilsonville Run 259 was made in a two-stage continuous unit with catalyst in both reactors. The feed coal was Ireland Mine, Pittsburgh seam bituminous coal. The sampling point from which the individual samples were obtained is called "Interstage" and was located between the first and second reactors. The sample designator for these samples is R1235. Each individual sample was distilled to an atmospheric equivalent boiling point of $850{ }^{\circ} \mathrm{F}$. Approximately half of the $850^{\circ} \mathrm{F}^{+}$fractions were then combined to make the composite sample. An analysis of the composite is attached.

I shipped to you, under separate cover, $50 \mathrm{~g}$ of the composite sample. We retained an additional limited quantity. Please contact me or Dick Winschel (412-854-6683) if you wish to obtain more of this sample or other samples. Please let me know if I can otherwise help by providing additional information. We welcome any comments you wish to make on the progress of your work with this sample.

Sincerely,

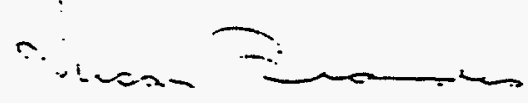

S. D. Brandes

Sr. Research Engineer

Is

cC: R. M. Statnick

R. A. Winschel

G. A. Robbins

M. A. Nowak - DOE/FETC 


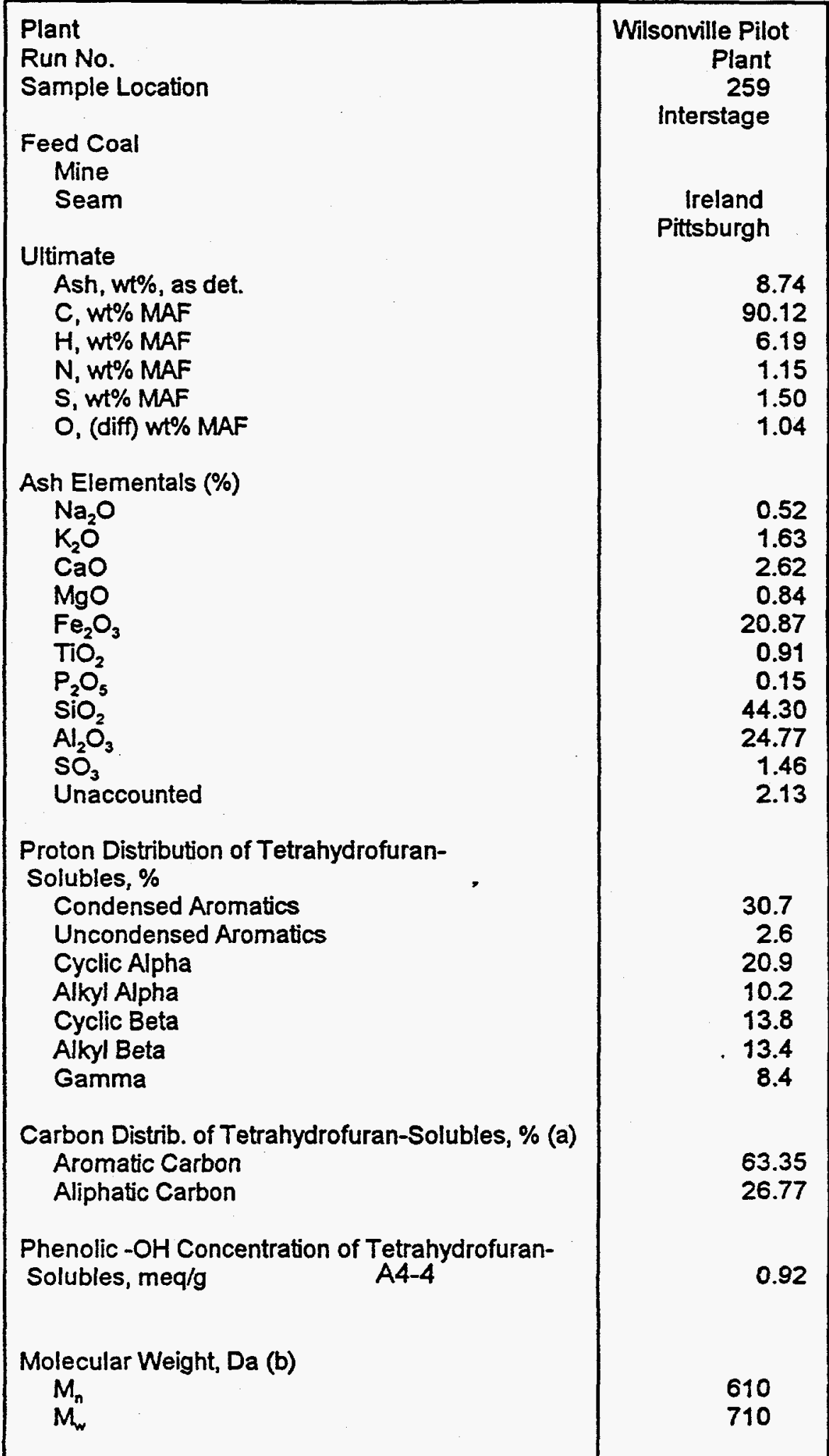

a) Carbon distribution determined by Western Research institute using solid-state ${ }^{13} \mathrm{C}-\mathrm{NMR}$

b) Molecular weight data determined by SRI International using field ionization mass spectrometry (FIBS) 
April 18, 1997

CONSOL Ine.

Research \& Development 4000 Brownsville Road

Library, PA 15129-9566

412.854 .6600

FAX: $412.854-6613$

412-854-6683

Mr. Richard Sprecher

U.S. Department of Energy

Federal Energy Technology Center

P.O. Box 10940

Pittsburgh, PA 15236-0940

Subject: DOE Contract DE-AC22-94PC93054

\section{Dear Rich:}

This is to document the samples you picked up on April 18 from CONSOL. The eleven samples were contained in $20 \mathrm{~mL}$ vials. These samples are aliquots of the materials returned to CONSOL from Caleb Brett. Caleb Brett, under subcontract to CONSOL, conducted crude oil assays on two net products of HTI Run PB-03. The one product, HTI PB-03-6,7,8 was produced with the hydrotreater on line. 'The second product, HTI Run PB-03-9,10,11 was produced while the hydrotreater was by-passed. As part of the crude oil assay, Caleb Brett fractionally distilled both samples. One of the crude oils and most of the fractions were not completely consumed in the assay tests. I provided you with samples of the available crude and all available fractions, as listed below.

\section{Sample}

HTI PB-03-6,7,8

"

"

"

"

HTI PB-03-9, 10,11

\section{Fraction Boiling Point, ${ }^{\circ} \mathrm{F}$}

$70-180$

180-350

400-550

550-650

$650^{+}$

crude oil

$70-180$

180-350

400-550

$550-650$

$650^{+}$

My understanding is that you will analyze these materials by high resolution mass spectrometry to determine the susceptibility of various nitrogen-compound types to hydrotreating. This should be a good set of samples for that purpose, as long as your method has the sensitivity to handle the low nitrogen contents of the hydrotreated fractions. Details of CONSOL's and Caleb Brett's 
work with these materials, including detailed analyses, can be found in the quarterly Technical Progress Report for June through September 1996 under the subject contract. Please contact Gary Robbins if you would like a copy of the draft report.

Sincerely.<smiles>CCCCCCC</smiles>

R. A. Winschel

Research Group Leader

Exploratory Research Group

Is

cc: M. A. Nowak

F.P. Burke

R. M. Statnick

P. Zhou - BRSC 


\title{
APPENDIX 5
}

\section{FORMAT REQUEST FOR SOFTWARE OF KINETIC/MECHANISTIC MODEL OF RESID REACTIVITY}

\author{
Letter from S. D. Brandes to the University of Delaware
}


March 6, 1997

CONSOL Inc.

Research \& Development 4000 Brownsville Road

Library. PA 15129.9566

$+13.854-6600$

F.t. $412-354.6613$

$412-854-6568$

\section{Darin Campbell}

Department of Chemical Engineering

Colburn Laboratory

University of Delaware

Newark, DE 19716-3110

\section{Subject: Subcontract Under DOE Contract DE-AC22-94PC93054}

\section{Dear Darin:}

I spoke with Mike Nowak, our DOE contracting officer's representative, concerning the form in which the kinetic/mechanistic model of resid reactivity you are developing is to be delivered to DOE.

The following items are to be submitted: 1) an electronic copy of the model either on disc or tape; 2) a hard copy printout of the code; 3 ) documentation which describes the software; specifically, what it is intended to do and how it is expected to do it; and 4) an operating manual that is sufficiently detailed that a knowledgeable individual can use the model.

I believe, based on our conversation, that items 1 and 2 should be easily accomplished. I suggest that you keep item 3 to a maximum length of a page or two. Item 4 is important and may require more space.

When you are ready to deliver the software, please contact me. I will put you in touch with one of our systems analysis engineers who will be able to tell you the formats, etc. we are capable of employing.

If you have any questions, feel free to call me. Dick Winschel and I will be at Delaware on April 30th and look forward to a demonstration of the model at that time.

Sincerely,

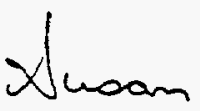

S.D. Brandes

Sr. Research Engineer

Ils

cC: F. P. Burke

R. M. Statnick

R. A. Winschel

W. H. Calkins - University of Delaware

M. T. Klein - University of Delaware

M. A. Nowak - DOE/PETC 


\section{APPENDIX 6}

\section{"KINETICS OF HYDROPROCESSING OF COAL-DERIVED VACUUM RESIDS"}

By: Shaojie Wang, He Huang, Keyu Wang, M. T. Klein and W. H. Calkins University of Delaware

Department of Chemical Engineering

Newark, DE 19716 


\title{
KINETICS OF HYDROPROCESSING OF COAL-DERIVED VACUUM RESIDS
}

\author{
Shaojie Wang, He Huang, Keyu Wang, M.T. Klein and W.H. Calkins* \\ Department of Chemical Engineering \\ University of Delaware \\ Newark, DE 19716
}

Key words: coal-derived resid, hydroprocessing, kinetics

\section{Introduction}

The direct liquefaction of coal produces a substantial amount of high boiling, nondistillable residuum, whose amount depends upon a number of factors such as the coal type, the hydrogen donor strength of the solvent, activity of the catalyst, and the conditions under which the direct liquefaction was run. Because of its high boiling point and potential thermal instability, this material is not suitable for processing in a conventional petroleum refinery. In a commercial liquefaction process as visualized today, therefore, this material would be recycled to the process to recover its energy value and to provide some of the solvent needed for the coal liquefaction process itself. Furthermore, this recycle oil has been shown to have a beneficial effect (i.e. increased oil yield) in the liquefaction process $(1,2)$. Thus, it became important to determine the rates of conversion of these residual materials to products boiling in the fuel range (e.g. $<850^{\circ} \mathrm{F}$ ) and to know whether these high boilers will build up or be rapidly broken down in the recycling process. It was to follow the rates of resid breakdown (resid reactivity) under conditions approximating the conditions in the liquefaction process that this program was undertaken. Knowing the rates of resid condensation as well as breakdown are also important as retrograde processes reduce product yields and foul catalysts and equipment. This required the use of a reactor system capable of measuring hydroprocessing rates at very short contact times and the development of analytical methods for measuring the conversion and boiling ranges of the products. Resid conversion rates (both condensation and breakdown) would be correlated with composition data obtained by other analytical methods (e.g. TGA, NMR, elemental analysis etc.)

\section{Experimental Section}

Apparatus. The design and operation of the Short Contact Time Batch Reactor (SCTBR) system have been described in detail elsewhere (3). In operation, both the empty $30 \mathrm{~cm}^{3}$ reactor and the preheater and precooler are immersed in a fluidized sand bath and brought up to reaction temperature. High pressure hydrogen gas provided the driving force to deliver the reaction mixture of solvent, coal and catalyst from a blow case into the reactor at reaction temperature in of the order of 0.3 seconds, eliminating the heat up limitations in kinetic measurements. Discharging and quenching of the reaction mixture was carried out in the similar time frame. Hydrogen bubbled through the reactor from the bottom provided the necessary agitation. Temperature control was within $\pm 2{ }^{\circ} \mathrm{C}$. Reaction times as short as 5 seconds could be measured with considerable precision. 
Materials Studied. Thirteen resid samples (boiling above $850^{\circ} \mathrm{F}$ ) from coal liquefaction runs made at the Wilsonville pilot plant and two resid samples from Hydrocarbon Research Institute bench scale unit were prepared and supplied by CONSOL Inc. The feed coals for the resids produced at the Wilsonville pilot plant were Wyodak-Anderson, Illinois \#6 and Pittsburgh coals. Selected properties, such as elemental analysis and the ${ }^{13} \mathrm{C}$ NMR patterns obtained by CONSOL Inc., of each resid are shown in Table 1.

Resid Conversion Reactions. All reactions were run as mixtures of tetralin $\mathrm{T}$ (the donor solvent) and resid $R$ over a range of $T / R$ ratios, temperatures and catalyst. For each reactor run, 5 - 10 grams of resid were used together with added tetralin to make up the desired $T / R$ ratio. Holdup of material prevented complete recovery of the reaction products. Recoveries varied from 75 to $85 \mathrm{wt} \%$, depending upon the $T / R$ ratio used. The determination of conversion and subsequent analytical results were therefore based on representative aliquots. Molybdenum naphthenate was used as the catalyst and was sulfided in-situ using methyldisulfide.

Reaction Product Workup Procedure. The reaction products were worked up by separating the solids from the liquids by filtration (Figure 1). The solid filter cake was washed with methylene chloride which went into the filtrate with the product liquids. The filtrate was then distilled at low temperatures $\left(45^{\circ} \mathrm{C}\right)$ to remove the methylene chloride. The resulting solid cake and the filtrate were analyzed separately.

Analytical Methods - Conversion. The conversion to liquid was determined using thermogravimetric analysis (TGA) on the solid cake by an ash balance calculation.

The tetralin content of the methylene chloride-free filtrate was determined by gas chromatography using an added 1-methylnaphthalene internal standard.

To determine the amount of liquid product boiling above and below $850^{\circ} \mathrm{F}\left(454^{\circ} \mathrm{C}\right)$, a boiling range method, SimDis TG, was developed based on TGA (4).

The conversion of resid to the material boiling below $850^{\circ} \mathrm{F}$ was estimated by Equation $1:$

$$
\text { Conversion }\left(<850^{\circ} F\right)=\operatorname{TSF} \times\left(1-\frac{850^{\circ} F^{*}}{R S F}\right)
$$

where TSF is the Tetralin Soluble Fraction of the resid (daf basis) determined by ash content in the solid resid after resid hydroprocessing; RSF is the Resid Soluble Fraction in tetralin and 850 ${ }^{\circ} \mathrm{F}^{+}$is the fraction boiling above $850^{\circ} \mathrm{F}$.

Analytical Methods - Resid Characterization. The resids studied were characterized by thermogravimetric analysis at $10^{\circ} \mathrm{C} / \mathrm{min}$ in nitrogen from room temperature to $600^{\circ} \mathrm{C}$. This was followed by combustion of the remaining organic material at $100^{\circ} \mathrm{C} / \mathrm{min}$ to $850^{\circ} \mathrm{C}$ in air. The derivative DTG curves, Volatile Matter (VM), Fixed Carbon (FC), and ash were determined. These TGA parameters as well as the peak temperatures and peak heights from the DTG curves are also included in Table 1.

\section{Results and Discussion}

As discussed in a previous section of this paper, conversion has been determined in part by an ash balance. Efforts to carry out hydroprocessing of resids using the Ni/Mo on alumina catalyst used in Wilsonville, however, resulted in unreliable conversions data because of the large amount of ash in the catalyst. In addition, it was found that the supported catalyst changed 
as the hydroprocessing progressed, making the calculations of conversion unreliable. Sulfided molybdenum catalyst, on the other hand, contributed little ash (which can be corrected for) to the system and gave very reproducible results. Preliminary experiments using a range of sulfided molybdenum naphthenate catalyst concentrations from $0.9 \mathrm{wt} \%$ to $5.0 \mathrm{wt} \%$ showed that $0.9 \mathrm{wt} \%$ catalyst resulted in only a barely detectable increase in conversion over uncatalyzed runs. However, 3 to $5 \mathrm{wt} \%$ (based on the resid) gave significant conversion to lower boiling products.

After considerable experimentation to determine appropriate reaction conditions, all 15 resids were hydroprocessed for 30 minutes at $420^{\circ} \mathrm{C}$ in 3 to 1 tetralin to resid weight ratio and 1500 psig hydrogen with and without sulfided molybdenum naphthenate catalyst (as 3 wt\% molybdenum based on the resid charged). Each resid was also run at ambient temperature for comparative purposes.

Tables 2 and 3 show the conversions to material boiling below $850^{\circ} \mathrm{F}$ for the thermal and catalyzed hydroprocessed resids, respectively. It is to be noted that significant conversion to lower boiling material occurs even in the absence of catalyst. However, in the presence of the molybdenum catalyst, conversion to the lower boiling material was at least doubled. To attain as much as 30 to $40 \%$ conversion requires a significant amount of catalyst.

As Tables 2 and 3 show, there is considerable variation among the resids in terms of their reactivity and convertability to lower boiling products. Plots of the thermal and catalyzed conversions of the resids vs the feed coal types are shown in Figure 2. In the thermal hydroprocessing, there appears to be a correlation with the coal type used in the liquefaction, i.e., the lower rank coal produced resid which gave higher conversion on hydroprocessing in the absence of catalyst. On the other hand, if a catalyst is used, the resids from the three coals studied showed little or no difference in conversion under the conditions used. It will be noted in Figure 3 and Tables 1, 2 and 3 that those resids having high DTG peaks and high aromatic carbon content (by ${ }^{13} \mathrm{C}$ NMR) generally show low conversions under the thermal hydroprocessing conditions while lower aromatic carbon containing resids show higher conversions in thermal hydroprocessing. Use of a strong catalyst apparently compensates in part for the high aromaticity.

SimDis TGA on the solid filter cake showed that, whereas there is considerable solubility of the resid in tetralin, up to $80 \mathrm{wt} \%$, the solids themselves are not degraded to lower boiling material. Therefore, the resid must be solubilized in the recycle solvent for the resid breakdown to occur.

\section{Summary and Conciusions}

With the appropriate catalyst and conditions approximating coal liquefaction, high boiling coal-derived resids do break down to lower boiling products as they are recycled to the coal liquefaction process.

Coal-derived resids vary widely in their reactivity toward breakdown to lower boiling products under both thermal and catalytic conditions.

High catalyst activity appears to be necessary to convert these refractory materials to lower boiling materials.

Solubilization of the resid in the processing solvent is necessary for the molecular breakdown. 


\section{Acknowledgements}

The support of various portions of this work by the Department of Energy and CONSOL Inc. under subcontract DE-AC22-94PC93054 is acknowledged. The guidance and assistance of Drs F.P. Burke, R.A. Winschel and S.D. Brandes in providing samples, analytical data and other assistance were also major factors in the progress of this project.

\section{References}

1. Whitehurst, D.D.; Mitchell, T.O.; Farcasiu, M. Coal Liquefaction the Chemistry and Technology of Thermal Processes Academic Press: New York, 1980.

2. Grint, A.; Jackson, W.R.; Larkins, F.P.; Louey, M.B.; Marshall, M.; Trewhalla, M.J.; Watkins, I.D. Fuel 1994, 73, 381.

3. Huang, H.; Calkins, W.H.; and Klein, M.T. Energy \& Fuels 1994, 8, 1304.

4. Huang, H.; Wang, K.; Wang, S.; Klein, M.T.; and Calkins, W.H. ACS Fuel Chem. Div. Prep. 40, (3) 485, 1995. 
Table 1 Selected properties of the resids

\begin{tabular}{|c|c|c|c|c|c|c|c|c|c|}
\hline Resid & Feed Coal & Number & Stream* & $f_{a}$ & $\begin{array}{l}\text { VM } \\
\text { wt\% }\end{array}$ & $\begin{array}{l}\mathrm{FC} \\
\mathrm{wt} \%\end{array}$ & $\begin{array}{l}\text { Ash } \\
\text { wt \% }\end{array}$ & $\begin{array}{l}\text { Tpeak } \\
{ }^{\circ} \mathrm{C}\end{array}$ & $\begin{array}{l}\text { Peak Height } \\
w t \% / \min (\mathrm{daf})\end{array}$ \\
\hline Resid L & \multirow{6}{*}{$\begin{array}{l}\text { Wyodak-Anderson } \\
\text { Black Thunder }\end{array}$} & 4 & V 1067 & 34.0 & 51.3 & 48.7 & 19.1 & 470.7 & 4.59 \\
\hline Resid K & & 5 & R 1235 & 24.6 & 53.6 & 46.4 & 17.2 & 464.8 & 4.60 \\
\hline Resid H & & 6 & $\mathrm{~V} 131 \mathrm{~B}$ & 33.3 & 57.1 & 42.9 & 15.2 & 475.3 & 4.67 \\
\hline Resid F & & 10 & V 1067 & 24.3 & 55.2 & 44.8 & 17.5 & 461.5 & 4.36 \\
\hline Resid E & & 11 & R 1235 & 26.0 & 53.4 & 46.6 & 15.6 & 454.4 & 4.24 \\
\hline Resid G & & 12 & V 131B & 25.9 & 55.7 & 44.3 & 15.9 & 462.2 & 5.02 \\
\hline Resid I & \multirow{3}{*}{$\begin{array}{l}\text { Illinois No. } 6 \\
\text { Burning Star No. } 2\end{array}$} & 7 & V 1067 & 30.4 & 61.5 & 38.5 & 15.9 & 480.1 & 6.44 \\
\hline Resid M & & 8 & R 1235 & 29.4 & 59.7 & 40.3 & 13.7 & 481.8 & 6.16 \\
\hline Resid D & & 9 & V 131B & 29.2 & 70.9 & 29.1 & 9.9 & 490.5 & 6.83 \\
\hline Resid J & \multirow{3}{*}{$\begin{array}{l}\text { Pittsburgh } \\
\text { Ireland }\end{array}$} & 1 & V 1067 & 31.6 & 57.6 & 42.4 & 10.2 & 490.1 & 7.20 \\
\hline Resid B & & 2 & R 1235 & 33.3 & 61.1 & 38.9 & 8.7 & 493.6 & 7.43 \\
\hline Resid C & & 3 & V 131B & 31.5 & 61.0 & 39.0 & 8.5 & 490.2 & 6.23 \\
\hline Resid A & & & & 38.9 & 51.8 & 48.2 & 17.0 & 472.1 & 4.75 \\
\hline Resid N & & & & 20.6 & & & 0.4 & & \\
\hline Resid O & & & & 18.8 & : & & 4.1 & & \\
\hline
\end{tabular}

*

V $1067=$ interstage stream

$R \mathbf{1 2 3 5}=2$ 2nd stage product stream

$V 131 \mathrm{~B}=$ recycle stream 
Table 2 Conversion of thermal hydroprocessing of resid

\begin{tabular}{|c|c|c|c|c|c|c|c|c|}
\hline \multicolumn{3}{|c|}{ Resid } & \multicolumn{2}{|c|}{ Solid Residue } & \multicolumn{3}{|c|}{$\begin{array}{r}\text { Liquid Residue } \\
\end{array}$} & \multirow[t]{2}{*}{ Conversion to $850 \mathrm{~F}$ - } \\
\hline \multicolumn{2}{|c|}{ Sample } & Ash & Ash & TSF & Tetralin & SRF & $850 \mathrm{~F}+$ & \\
\hline Resid A & W258V-131B & 17.0 & 50.1 & 79.7 & 88.2 & 11.8 & 9.6 & 15.0 \\
\hline Resid B & W259R-1235 & 8.7 & 33.5 & 81.0 & 88.0 & 12.0 & 9.6 & 15.9 \\
\hline Resid C & W259V-131B & 8.5 & 35.0 & 82.7 & 86.2 & 13.8 & 10.8 & 17.7 \\
\hline Resid D & W261V-131B & 9.9 & 45.8 & 87.1 & 87.9 & 12.1 & 9.7 & 17.1 \\
\hline Resid E & W262R-1235 & 15.6 & 43.5 & 76.0 & 88.4 & 11.6 & 8.6 & 19.6 \\
\hline Resid F & W262V-1067 & 17.5 & 47.8 & 76.9 & 87.4 & 12.6 & 9.7 & 17.7 \\
\hline Resid G & W262V-131B & 15.9 & 46.5 & 78.3 & 88.7 & 11.3 & 8.7 & 18.2 \\
\hline Resid H & $\mathrm{W} 260 \mathrm{~V}-131 \mathrm{~B}$ & 15.2 & 46.6 & 79.5 & 87.7 & 12.3 & 9.0 & 21.3 \\
\hline Resid I & W261V-1067 & 15.9 & 50.8 & 81.8 & 88.9 & 11.1 & 8.5 & 19.4 \\
\hline Resid J & W259V-1067 & 10.2 & 38.7 & 82.0 & 86.4 & 13.6 & 11.5 & 12.9 \\
\hline Resid K & W260R-1235 & 17.2 & 49.0 & 78.4 & 86.9 & 13.1 & 9.8 & 19.7 \\
\hline Resid L & W260V-1067 & 19.1 & 51.3 & 77.6 & 89.2 & 10.8 & 8.3 & 17.8 \\
\hline Resid M & W261R-1235 & 13.7 & 45.6 & 81.1 & 90.1 & 9.9 & 7.9 & 16.0 \\
\hline Resid N & HTI POC-01, $0-43$ & 0.4 & 33.0 & 99.2 & 80.6 & 19.4 & 14.1 & 27.0 \\
\hline Resid O & HTI POC- $02,0-43$ & 4.1 & 38.0 & 93.1 & 70.6 & 29.4 & 22.4 & 22.1 \\
\hline & & & & & & & & \\
\hline \multicolumn{9}{|c|}{ Thermal: $420 \mathrm{C} ; 30 \mathrm{~min} ; 1500 \mathrm{psig} \mathrm{H} 2$} \\
\hline \multicolumn{9}{|c|}{ Catalytic: $420 \mathrm{C} ; 30 \mathrm{~min} ; 1500 \mathrm{psig} \mathrm{H} 2 ; 3 \mathrm{wt} \% \mathrm{Mo}$} \\
\hline \multicolumn{9}{|c|}{\begin{tabular}{|l|l|} 
Control: $25 \mathrm{C} ; 10 \mathrm{~min} ; 1500 \mathrm{psig} \mathrm{H} 2$ & \\
\end{tabular}} \\
\hline \multicolumn{9}{|c|}{ TSF: Tetralin Soluble Fraction of resid, wt\% (daf basis) } \\
\hline \multicolumn{9}{|c|}{\begin{tabular}{|l|l} 
RSF: Resid Soluble Fraction in tetralin, wt $\%$ & \\
\end{tabular}} \\
\hline \multicolumn{3}{|c|}{$850 \mathrm{~F}+$ : fraction of boiling above $850 \mathrm{~F}$} & & & & & & \\
\hline
\end{tabular}


Table 3 Conversion of catalytic hydroprocessing of resid

\begin{tabular}{|c|c|c|c|c|c|c|c|c|}
\hline \multicolumn{3}{|c|}{ Resid } & \multicolumn{2}{|c|}{ Solid Residue } & \multicolumn{3}{|c|}{ Liquid Residue } & \multirow[t]{2}{*}{ Conversion } \\
\hline Samp & Name & Ash & Ash & SF & Tetralin & SR in Tetralin & $850 \mathrm{Ft}$ & \\
\hline Resid A & W258V-131B & 17.0 & 51.5 & 80.7 & 82.5 & 17.5 & 9.9 & 35.0 \\
\hline Resid B & W259R-1235 & 8.7 & 40.0 & 85.6 & 79.8 & 20.2 & 11.2 & 38.1 \\
\hline Resid C & W259V-131B & 8.5 & 41.4 & 86.8 & 81.4 & 18.6 & 11.5 & 33.1 \\
\hline Resid D & W261V-131B & 9.9 & 54.9 & 91.0 & 77.9 & 22.1 & 14.1 & 32.9 \\
\hline Resid E & W262R-1235 & 15.6 & 44.2 & 76.6 & 80.6 & 19.4 & 11.3 & 32.1 \\
\hline Resid F & W262V-1067 & 17.5 & 49.4 & 78.3 & 79.6 & 20.4 & 10.7 & 37.4 \\
\hline Resid G & W262V-131B & 15.9 & 48.3 & 79.8 & 79.2 & 20.8 & 11.5 & 35.8 \\
\hline Resid H & W260V-131B & 15.2 & 50.9 & 82.7 & 75.6 & 24.4 & 11.3 & 44.6 \\
\hline Resid I & W261V-1067 & 15.9 & 56.6 & 85.5 & 76.2 & 23.8 & 11.9 & 42.7 \\
\hline Resid J & W259V-1067 & 10.2 & 43.8 & 85.4 & 76.5 & 23.5 & 11.9 & 42.1 \\
\hline Resid K & W260R-1235 & 17.2 & 52.1 & 80.8 & 78.1 & 21.9 & 12.4 & 35.2 \\
\hline Resid L & W260V-1067 & 19.1 & 53.5 & 79.5 & 79.1 & 20.9 & 11.7 & 35.0 \\
\hline Resid M & W261R-1235 & 13.7 & 53.7 & 86.3 & 80.2 & 19.8 & 11.7 & 35.3 \\
\hline Resid N & HTI POC-01, O-43 & 0.4 & 36.4 & 99.3 & 67.9 & 32.1 & 16.7 & 47.6 \\
\hline Resid 0 & HTI POC-02, O-43 & 4.1 & 48.3 & 95.4 & 70.2 & 29.8 & 19.1 & 34.3 \\
\hline \multicolumn{9}{|c|}{ Thermal: $420 \mathrm{C} ; 30 \mathrm{~min} ; 1500$ psig H2 } \\
\hline \multicolumn{9}{|c|}{ Catalytic: $420 \mathrm{C} ; 30 \mathrm{~min} ; 1500$ psig H2; $3 \mathrm{wt} \% \mathrm{Mo}$} \\
\hline \multicolumn{9}{|c|}{\begin{tabular}{|l|l|} 
Control: $25 \mathrm{C} ; 10 \mathrm{~min} ; 1500$ psig H2 & \\
\end{tabular}} \\
\hline \multicolumn{9}{|c|}{ TSF: Tetralin Soluble Fraction, wt $\%$ (daf basis) } \\
\hline \multicolumn{9}{|c|}{ RSF: Resid Soluble Fraction in tetralin, wt $\%$} \\
\hline $850 \mathrm{~F}+:$ fra & tion of boiling above & $50 \mathrm{~F}$ & & & & & & \\
\hline
\end{tabular}




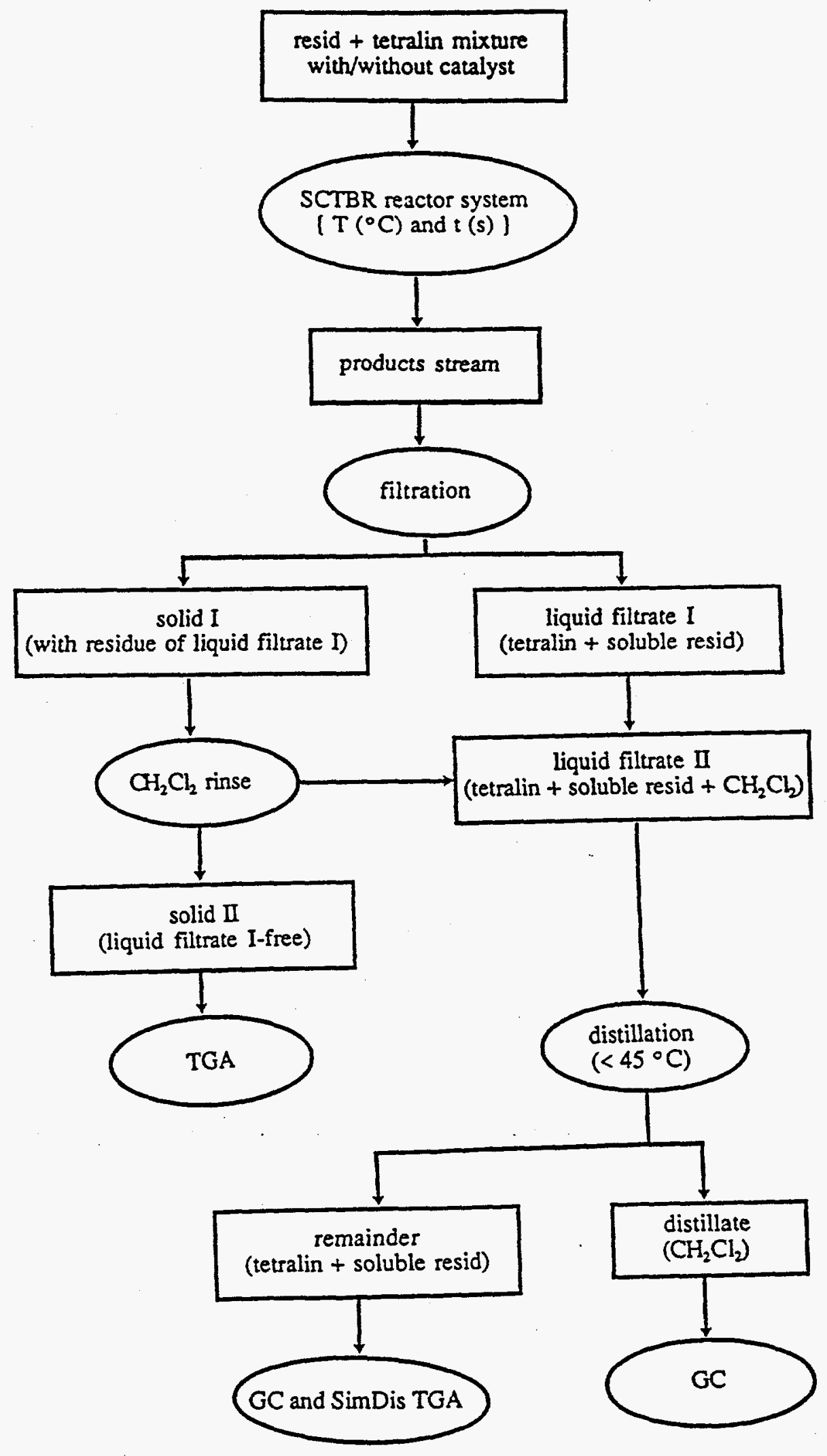

Figure 1 Scheme of the reaction product workup procedure 


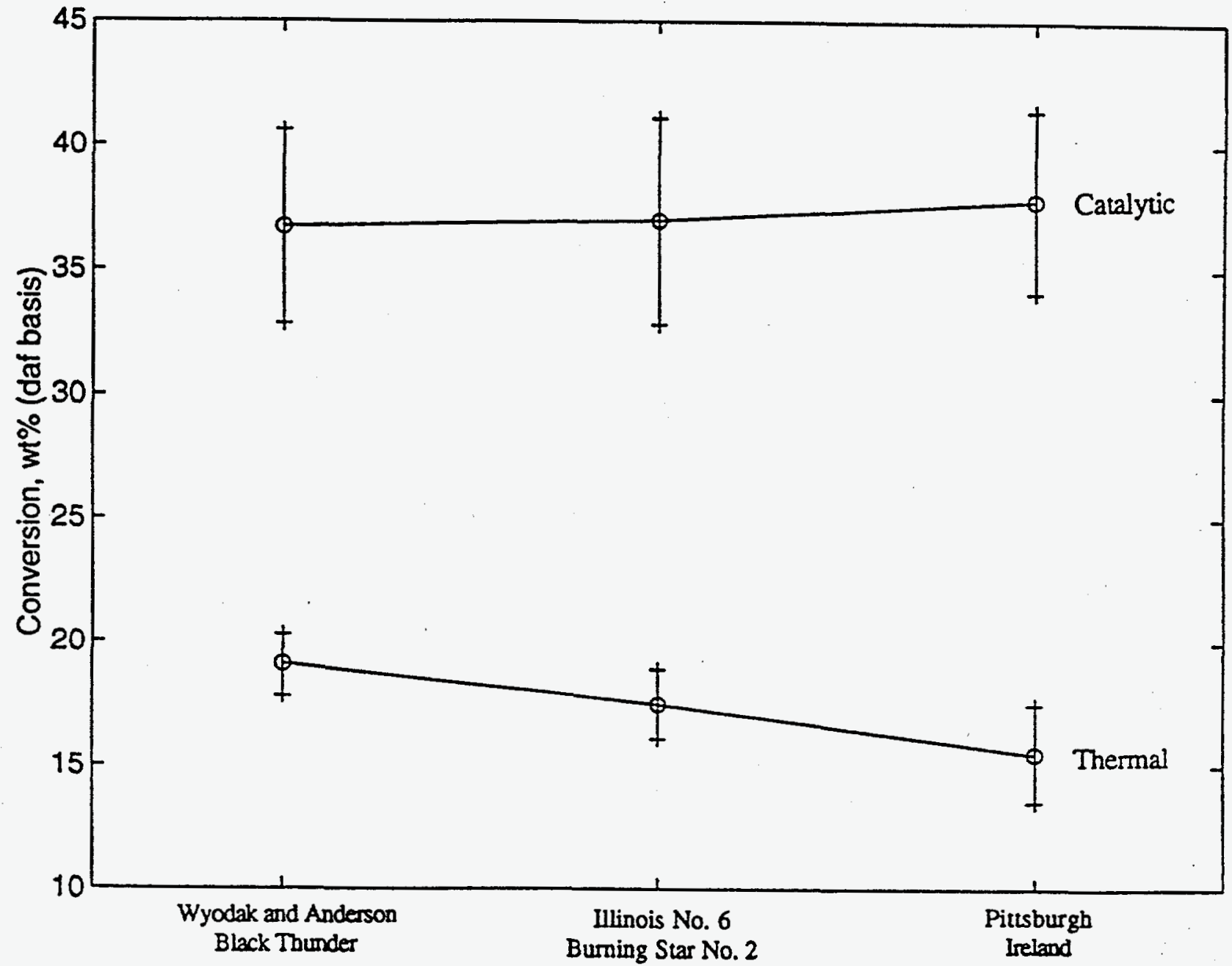

Feed Coal

Figure 2 Thermal and catalyzed hydroprocessing conversions of the resids vs feed coal type 


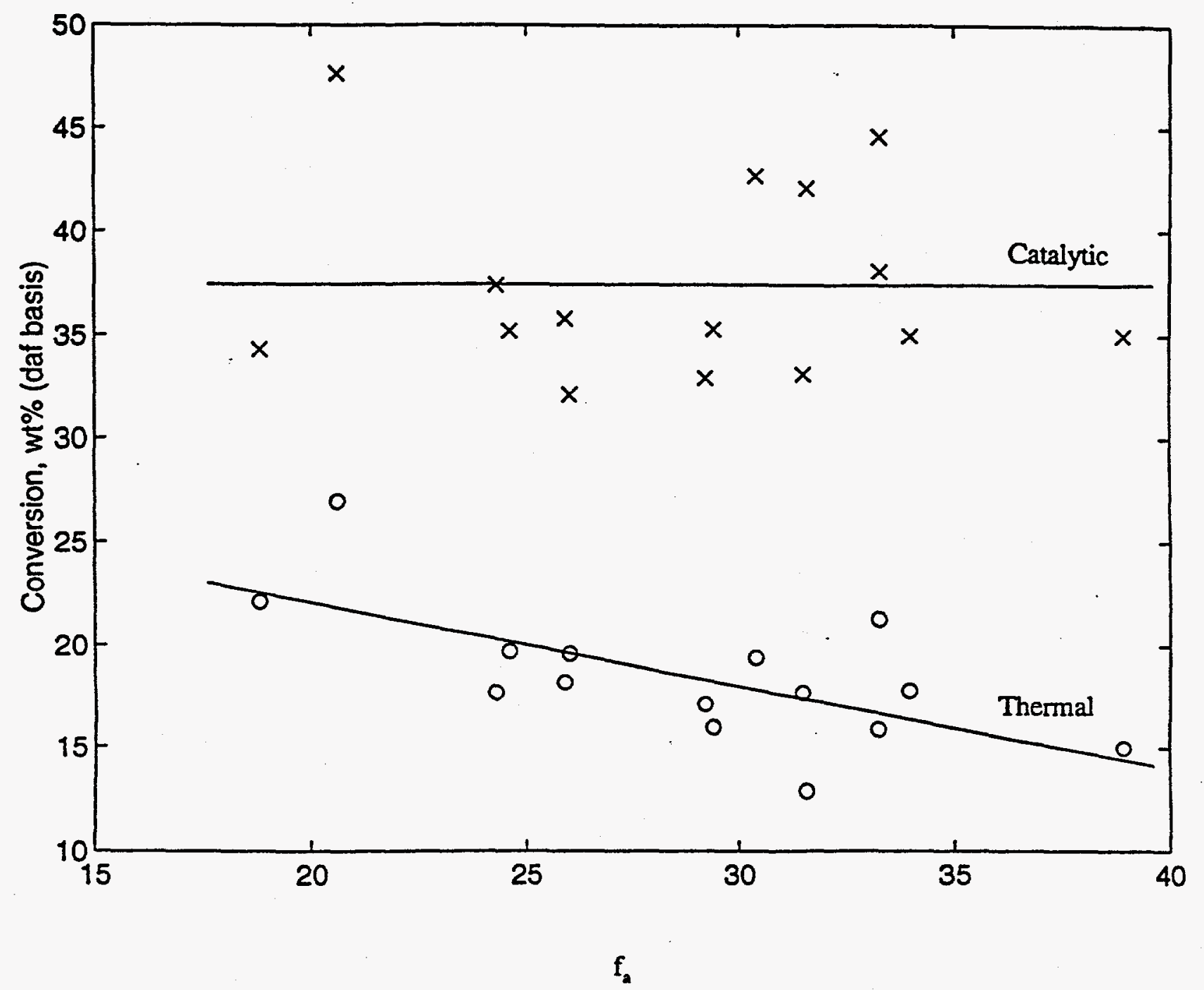

Figure 3 Thermal and catalyzed hydroprocessing conversions vs $f_{a}$ of the resids 